\title{
Information Requisition is the Core of Guideline-Based Medical Care: Which Information is Needed for Whom?
}

\author{
DIPLOMARBEIT
}

zur Erlangung des akademischen Grades

\section{Magistra der Sozial- und Wirtschaftswissenschaften \\ im Rahmen des Masterstudiums \\ Informatikmanagement \\ eingereicht von}

\section{Dipl.-Ing.techn. Theresia Gschwandtner}

Matrikelnummer 9949197

an der

Fakultät für Informatik der Technischen Universität Wien

Betreuung:

Betreuerin: ao.Univ.Prof. Mag. Dr. Silvia Miksch

Mitwirkung: Mag. Dr. Katharina Kaiser 



\section{Zusammenfassung}

Ein funktionelles Softwareprodukt kann nur dann entworfen werden, wenn zuvor genau untersucht wurde, (1) wer die Software verwenden wird, (2) welche Aufgaben er/sie damit erledigen will, und folglich, (3) welche Informationen notwendig sind, um diese Aufgaben bestmöglich zu unterstützen. Die Modellierung und Anwendung einer computer-ausführbaren klinischen Leitlinie involviert viele verschiedene Aufgaben. Diese Aufgaben reichen von der Modellierung des medizinischen Wissens in eine computer-interpretierbare Form bis zur eigentlichen Ausführung und Anwendung der klinischen Leitlinie bei der PatientInnenversorgung. Folglich betreffen sie eine Reihe von verschiedenen Personengruppen, nämlich InformatikerInnen, MedizinerInnen, Pflegepersonal und PatientInnen. Der Entwurf eines Softwareprodukts, das all diese Aufgaben effizient unterstützt, ist daher noch immer eine Herausforderung.

Eine sorgfältige Untersuchung der verschiedenen Benutzergruppen der Software sowie der verschiedenen Aufgaben, die mit Hilfe der Software erledigt werden sollen, ist daher unabdingbar, um festzulegen, welche Informationen und Funktionen die Software anbieten muss.

Wir haben im Rahmen dieser Arbeit eine umfassende Literaturrecherche durchgeführt. Dabei haben wir einerseits die verschiedenen Aufgaben bei der Modellierung einer klinischen Leitlinie in eine computer-ausführbare Form untersucht, andererseits die Informationsbedürfnisse von ÄrztInnen und Pflegepersonal, sowie die Informationsbedürfnisse von PatientInnen. Im Folgenden haben wir diese Informationen festgelegt und kategorisiert, um einen verlässlichen Ausgangspunkt für den Entwurf und die Entwicklung einer geeigneten Software für die Modellierung und Anwendung einer computer-ausführbaren klinischen Leitlinie zu schaffen. 



\begin{abstract}
One indispensable precondition for designing a functional software product is to gather knowledge about (1) who is going to use the software, (2) which tasks does he/she want to perform with its help, and consequently, (3) which information is necessary to best support these tasks. Since the modeling and execution of a computerized clinical practice guideline is composed of numerous tasks and because a range of different stakeholders are involved - knowledge engineers, medical experts, nurses, and patients -, the design of a tool to support the whole life cycle of a computerized clinical guideline, i.e., from modeling the medical knowledge into a computerinterpretable representation to its actual execution in clinical care, is still a challenging issue. To this end, a comprehensive investigation of the user groups and the issues they encounter is a prerequisite to decide about the information and features that should be provided by the software.

In the context of this thesis we have conducted a comprehensive literature study about the different tasks involved in modeling a clinical practice guideline into a formal representation as well as about the information needs of caregivers, i.e., physicians and nurses, and last but not least the information needs of patients. We have assessed and categorized the above mentioned information required for each task and user group in order to create a reliable starting point for the development of a functional software tool for the modeling process and execution of a computerized clinical practice guideline.
\end{abstract}





\section{Acknowledgments}

I would like to thank my advisors, Silvia Miksch and Katharina Kaiser, for supporting and helping me while working on this thesis. Moreover, I want to thank them for the friendly atmosphere, for enabling comfortable conditions for my work, and for putting a lot of trust in me, which I do not take for granted.

In addition - and most importantly - I want to thank my father, Helfried, for the great support during all the years of my studies, but especially for helping and encouraging me while working on this thesis. He did not only a great job in extensively proofreading this thesis, but also lifted me up when I was exhausted.

The research leading to these results has received funding from the European Community's Seventh Framework Programme (FP7/2007-2013) under grant agreement no. 216134. 



\section{Contents}

$\begin{array}{ll}\text { Contents } & \text { ix }\end{array}$

1 Introduction 1

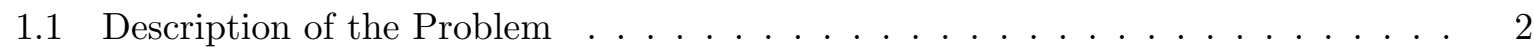

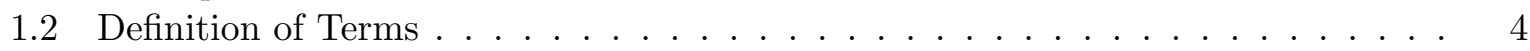

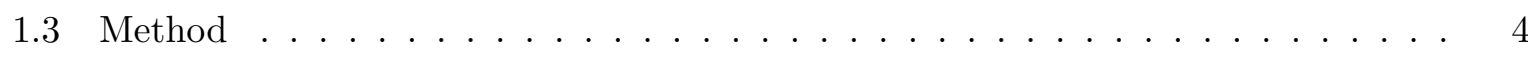

2 Literature Research $\quad 7$

2.1 Different User Groups . . . . . . . . . . . . . . . . . . . . . . . . . . 8

2.2 Modelers' Tasks . . . . . . . . . . . . . . . . . . . . . . . . . 11

2.2.1 Components of Guideline Representation Languages . . . . . . . . . . . . 11

2.2.2 Formalization Process . . . . . . . . . . . . . . . . . . 13

2.3 Clinical Information . . . . . . . . . . . . . . . . . . . . 28

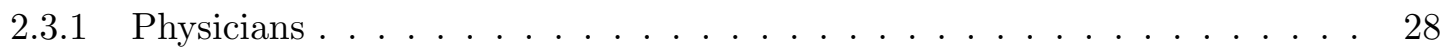

$2.3 .2 \quad$ Nurses . . . . . . . . . . . . . . . . . . . . . . . . . 34

2.3 .3 Patients . . . . . . . . . . . . . . . . . . . . . 38

3 Categorization of the Findings $\quad 45$

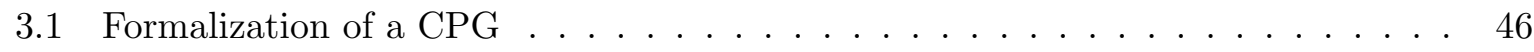

3.1.1 Model-Centric Formalization . . . . . . . . . . . . . . . 46

3.1 .2 Document-Centric Formalization . . . . . . . . . . . . . . . 47

3.2 Clinical Information Needs . . . . . . . . . . . . . . . . . . 52

3.2.1 Physicians' Information Needs . . . . . . . . . . . . . . . . . . 52

3.2 .2 Nurses' Information Needs . . . . . . . . . . . . . . . . . . . . . . . . . . 56

3.2.3 Patients' Information Needs . . . . . . . . . . . . . . . . . . . . . 59

3.2.4 Categorization of Clinical Information Needs . . . . . . . . . . . . 63

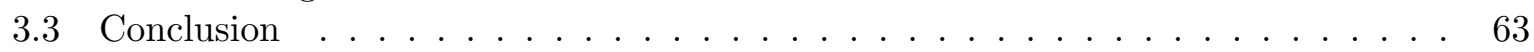

A Detailed Results of Studies $\quad 69$

A.1 Results of Studies on Physicians' Information Needs . . . . . . . . . . . . . . 69

A.2 Results of Studies on Nurses' Information Needs . . . . . . . . . . . . . . . 71

A.3 Results of Studies on Patients' Information Needs . . . . . . . . . . . . . . . . 74

$\begin{array}{ll}\text { Bibliography } & 77\end{array}$ 


\section{List of Tables}

2.1 Different terms used by diverse guideline modeling approaches [103] . . . . . . . 12

2.2 Different approaches of representing scheduling constrains, subplans of guidelines, and modeling of patient data $[103] \ldots \ldots \ldots \ldots . \ldots \ldots$

2.3 Different terms used by diverse guideline modeling approaches $[67,103]$. . . . . 13

2.4 Different guideline representation languages opposed to corresponding guideline

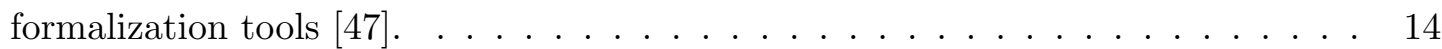

2.5 Major and minor categories of information needs according to Zeng and Cimino [108] (the frequency gives the percentage of this category of the total number of information needs verbalized during an observational study). . . . . . . . . .

2.6 Generic question patterns observed when thirteen physicians reviewed three clinical cases, representing patients with cardiovascular problems [77].

2.7 Recurring question patterns representing $47 \%$ of all questions asked by physicians while using a clinical information system (CIS) $[1] \ldots \ldots \ldots$. . . . . . . .

2.8 Summary of studies focusing on physicians' information needs between 1978 and 1994 [83] (the frequency gives the percentage of this information need of the total number of information needs detected during the study). . . . . . . . . . . . . 32

2.9 McCaughan et al. worked out a taxonomy of decisions made by nurses [55]. . . . 34

2.10 Information needs and communication difficulties perceived by nurses (McKnight

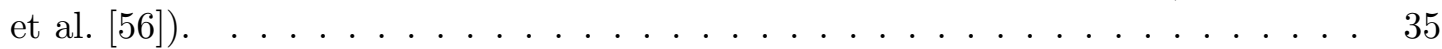

2.11 Frequency of generic questions identified by Xu et al. [107]. . . . . . . . . . . . 36

2.12 Most commonly asked questions by patients (Coulter et al. [15]) . . . . . . . . . 39

3.1 Categorization of document-centric formalization process (continued on next page). 47

3.1 Categorization of document-centric formalization process (continued on next page). 48

3.1 Categorization of document-centric formalization process (end) . . . . . . . . . 49

3.2 Unification of physicians' information needs (continued on next page). . . . . . . 52

3.2 Unification of physicians' information needs (end) . . . . . . . . . . . . . . 53

3.3 The most common generic question patterns of physicians' information needs $[1$, $14,26,27,77]$ (continued on next page). . . . . . . . . . . . . 54

3.3 Most common generic question patterns of physicians' information needs $[1,14$,

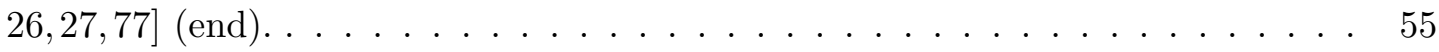

3.4 Unification of nurses' information needs (NPs: nurse practitioners) (continued on

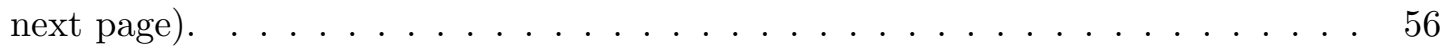

3.4 Unification of nurses' information needs (NPs: nurse practitioners) (continued). . 57

3.5 The most common generic question patterns relating to nurse practitioners' and nurses' information needs $[14,107] \ldots \ldots \ldots \ldots \ldots$. . . . . . . . . . . 58

3.6 Unification of patients' information needs (continued on next page). . . . . . . . 59

3.6 Unification of patients' information needs (continued on next page). . . . . . . . 60 
3.6 Unification of patients' information needs (continued on next page). . . . . . . . 61

3.6 Unification of patients' information needs (end) . . . . . . . . . . . . . 62

A.1 Most common generic questions derived from two studies in 1999 (103 family doctors) and 2000 (49 primary care doctors) by Ely et al. [26,27]. . . . . . . . . 69

A.2 Information needs and communication difficulties perceived by physicians (McKnight et al., $2002[56]) \ldots \ldots \ldots$. . . . . . . . . . . . . . 70

A.3 Frequency of generic question types asked by 6 medical residents (and average number by session) as described by Collins et al., 2009 [14] . . . . . . . . . . . . 70

A.4 Results of 134 questionnaires about the frequency of information needs of nurse practitioners' in North Carolina (Cogdill, 2003 [13]) . . . . . . . . . . . . . . 71

A.5 Results of 134 questionnaires about the frequency of information resource use by nurse practitioners' in North Carolina (Cogdill, 2003 [13]) . . . . . . . . . . . . 72

A.6 Frequency of information needs resulting from interviews of 20 nurse practitioners in North Carolina after their encounters with patients (Cogdill, 2003 [13]). . . . . 72

A.7 Frequency of generic question types asked by 4 nurse practitioners and 3 registered nurses from the New York Presbyterian Hospital while using a clinical information system (Collins et al., 2009 [14]). . . . . . . . . . . . . . . . . . . 73

A.8 Answers of 250 cancer patients in west Scotland to specific questions about their need for information (Meredith et al., 1996 [59]). . . . . . . . . . . . . . 75

A.9 Seven most popular sections of cancer information booklets chosen by 65 breast cancer patients (Jones et al., $2006[45]) \ldots \ldots \ldots \ldots$. . . . . . . 76

A.10 Seven most popular sections of cancer information booklets chosen by 29 prostate cancer patients (Jones et al., $2006[45]) \ldots \ldots \ldots$. . . . . . . 76 


\section{List of Figures}

2.1 Steps of the development cycle of a clinical practice guideline. . . . . . . . . . 9

2.2 SAGE: Steps in modeling clinical practice guidelines for integration into work-

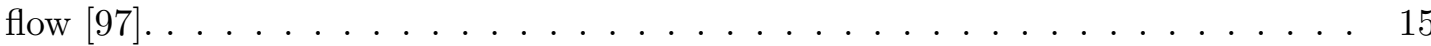

2.3 Part of an activity graph as encoded in the SAGE Guideline Model [97] (reproduction of the activity graph originally generated by means of the Protégé tool). 16

2.4 Screenshot of the GEM Cutter II editor. . . . . . . . . . . . . . . . . . . . 18

2.5 GEM: top level elements [60] . . . . . . . . . . . . . . . . . . . . . 19

2.6 Stepper: step-wise guideline formalization. . . . . . . . . . . . . . . . . 22

2.7 DELT/A - The two top panes show linked views of the same guideline. The top left view shows the textual guideline while the top right view shows its formal representation. The bottom pane is the macro view which provides macros to facilitate the creation of the formal guideline representation [101] . . . . . . . . . 24

3.1 Tasks of document centric guideline formalization (continued on next page). . . . 50

3.1 Tasks of document centric guideline formalization (end) . . . . . . . . . . . 51

3.2 Information on patient data. . . . . . . . . . . . . . . . . . 64

3.3 Information relevant for diagnosis. . . . . . . . . . . . . . . . 64

3.4 Information about a disease/condition. . . . . . . . . . . . . . . 65

3.5 Information on the management of the disease/condition. . . . . . . . . . . 66

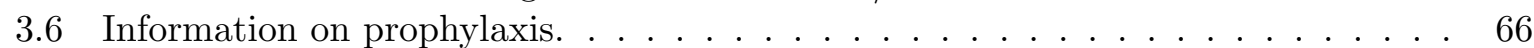

3.7 Information on support for the patient. . . . . . . . . . . . . . . . 67

3.8 Information about the impact of the condition on family members. . . . . . . . 67

3.9 Institution specific information. . . . . . . . . . . . . . . . 67

3.10 Information about other healthcare providers. . . . . . . . . . . . . . 67

3.11 References to important information. . . . . . . . . . . . . . . . . . 68 
Chapter 1

\section{Introduction}




\subsection{Description of the Problem}

In recent years different software systems have been developed to support the design and execution of clinical practice guidelines, each of them focusing on specific tasks $[19,67]$. From the creation of a computer-executable model of a clinical practice guideline to its actual implementation in clinical care a variety of tasks emerge, such as translating the medical knowledge of a textual clinical guideline, i.e., recommendations, into a computer-interpretable model, applying a recommended therapy on a patient, or answering clinical questions. Obviously, these tasks are to be performed by professionals of different categories, e.g., knowledge engineers, and physicians.

However, a guideline tool supporting all tasks emerging during the modeling and execution of a guideline would be of great benefit. In other words: a consistent environment from the formalization of the textual guideline to its actual implementation has important advantages. Existing guideline formalization approaches $[72,86,101]$ have stated the following minimal requirements of such a tool:

- providing links between the different models of the guideline, i.e., the textual guideline, the intermediate representations in the modeling process, and the computer interpretable model,

- backtracking given recommendations to their source in the original text and the other way round, if any parts of the guideline text are to be changed, and finally

- facilitating maintenance and modification of the guideline by applying corrections and changes in one model to all other models as well.

Hence, a guideline software system has to be designed to deal with all given tasks and user groups. There are several important aspects to be considered in developing such a tool. Nielsen has mentioned one important aspect, namely that the identification of possible different user groups, as well as understanding the tasks a user wants to perform by means of a software product and the information required for it is mandatory to make valid decisions about the design of the product [62].

This led us to investigate the following research questions: Main Question:

- Who are the different users of a computerized clinical guideline and which kinds of information have to be provided to best support their tasks?

Sub Questions:

- How can these information requirements be categorized?

- What kinds of information do guideline modelers need when creating a computer-executable model of a clinical practice guideline?

- What kinds of information do physicians and nurses need when executing of a guideline in clinical care?

- What kinds of information should be provided for patients?

This thesis therefore deals with a presentation and analysis of a number of representative research studies carried out in related fields - the formalization of a clinical practice guideline, information needs arising in clinical care, as well as information needs of patients - in order to 
establish a sound basis for the challenge of designing a software system supporting the modeling process and the application of such a guideline.

Several studies about the information needs during clinical care exist, e.g., surveys on the information needs of physicians $[1,12,14,26,27,36,56,77,83,108]$, of the nursing staff $[7,13,14$, $55,56,106,107]$, and of patients $[15,23,45,49,59,71,76,87,88,102]$. They are mostly concerned with information requirements not specifically tailored to which kinds of information should be provided in connection with the execution of a clinical guideline.

However, there are hardly any useful surveys on the information needs of guideline modelers to be found in available literature. To this end, the modelers' information needs have to be derived from the tasks involved in the development of a computerized clinical guideline. 


\subsection{Definition of Terms}

In the following subsections we specify the two terms "clinical practice guideline" and "protocol", which are essential for the understanding of this thesis.

\section{Computerized Clinical Practice Guidelines (CPGs)}

"Clinical practice guidelines are systematically developed statements to assist practitioner and patient decisions about appropriate health care for specific clinical circumstances."( [29], p.8)

Clinical practice guidelines provide recommendations on the appropriate treatment of specific conditions according to best available scientific evidence. Thus, they are important means for quality control, they facilitate clinical decision making, they establish standards to reduce inappropriate variation in health care, and they can be used for education and training of physicians $[24,29,98]$.

There are different ways to develop CPGs. Some CPGs are based on the opinion of experts in specific fields, others rely on a formal consensus process, and evidence-based guidelines rely on systematic review of clinical evidence [98].

Being intended to improve the quality of patient care and to reduce costs, CPGs are increasingly being used. However, CPGs provide population-based recommendations while clinician decision-making is most effectively supported by providing patient-specific advice delivered during patient encounters [67]. To this end, making CPGs computer-executable paves the way to guideline-based point-of-care decision support systems, which entail numerous benefits, for instance $[24,98]$ :

- Providing recommendations tailored to an individual patient;

- Not interrupting clinical workflow, by providing integrated computer support;

- Revealing inconsistencies and errors in the text of the guideline;

- Providing reminders and alerts.

\section{Protocols}

"Protocols are local tools that set out specifically what should happen, when and by whom in the care process. They can be seen as the local definition of a particular care process derived from a more discretionary guideline. They are in essence tools that assist in quality improvement and reducing inequalities. ... Protocols respect local circumstances, and variation will due to the differing types of local provision." [92]

Protocols differ from CPGs in that they are defined in greater detail and give precise instructions on what to do at specific institutions.

\subsection{Method}

We conducted a comprehensive literature review to investigate the information needs of different user groups of a computerized CPG. As a first step, we have examined the available literature to derive which user groups are actually involved in the development cycle of a CPG. Subsequently, 
we have focused our review on the different tasks involved in the modeling process of a clinical practice guideline as well as on the information needs of caregivers and patients.

We have examined several Internet resources for relevant scientific papers. On the one hand, we have looked for papers of specialized organizations and journals focusing on medicine (e.g., Medical Library Association [58], British Medical Journal [9]) and others focusing on medical informatics (e.g., American Medical Informatics Association [2], Artificial Intelligence in Medicine [3]). On the other hand, we have used general search engines (e.g., Google Scholar [35]) looking for scientific papers matching terms such as "clinical practice guideline", "nurse information needs", "clinical information needs", etc. In addition, we have checked the reference lists of relevant articles for additional information. 
Chapter 2

\section{Literature Research}




\subsection{Different User Groups}

In order to derive the different user groups involved in the development cycle of a CPG, we have examined the available literature with respect to articles describing the different tasks arising during the development of a computerized CPG.

Different research groups have investigated the development cycle of a CPG whith similar results. For instance, Quaglini et al. [69] suggest four development steps:

1. Guideline development: clinical experts and knowledge engineers work together to transform a narrative guideline into a formalized computer-executable model.

2. Verification of the logical correctness of the guideline model.

3. Guideline implementation: adaption of the formalized guideline to a specific organizational context in order to create a formalized site-specific guideline.

4. Use in daily practice: requires the representation of the guideline tasks and patient specific advice.

Moreover, Greenes et al. [37] also include the authoring process, and the encoding of eligibility criteria for a guideline:

1. Guideline authoring: create a guideline, store it in shared repositories, edit and maintain it over the network.

2. Guideline viewing: flowchart representation for visual navigation of the guideline.

3. Guideline modeling: specification for a structured grammar.

4. Guideline execution: object oriented representation for describing clinical actions and their parameters, generating patient-specific recommendations.

5. Encoding of eligibility criteria for a guideline.

Another important task - although not mentioned in these two articles - is the maintenance of the guideline. On the one hand, the fast progress of medical knowledge requires guidelines to be updated on a regular basis in order to present state-of-the-art knowledge [48], and, on the other hand, validation of a guideline model or its execution in clinical practice may reveal contradictions or erroneous instructions [101]. Thus, a software tool for the guideline development cycle has to provide means for efficiently up-dating the guideline and for keeping the textual guideline and its formal model consistent.

Based on these findings, we propose the six steps of a guideline development cycle (see Figure 2.1 for an algorithmic representation):

- Authoring: the process of creating a narrative clinical practice guideline;

- Modeling: translating the narrative guideline into a computer-executable formal representation model;

- Verification: verifying the logical correctness of the formal model,

- Adaptation: adapting the guideline model to a specific institution, 
- Execution: computer-executing the guideline in daily clinical practice (generating and applying recommendations for specific patients),

- Maintenance: keeping the guideline model up-to-date; correcting instructions of the guideline.

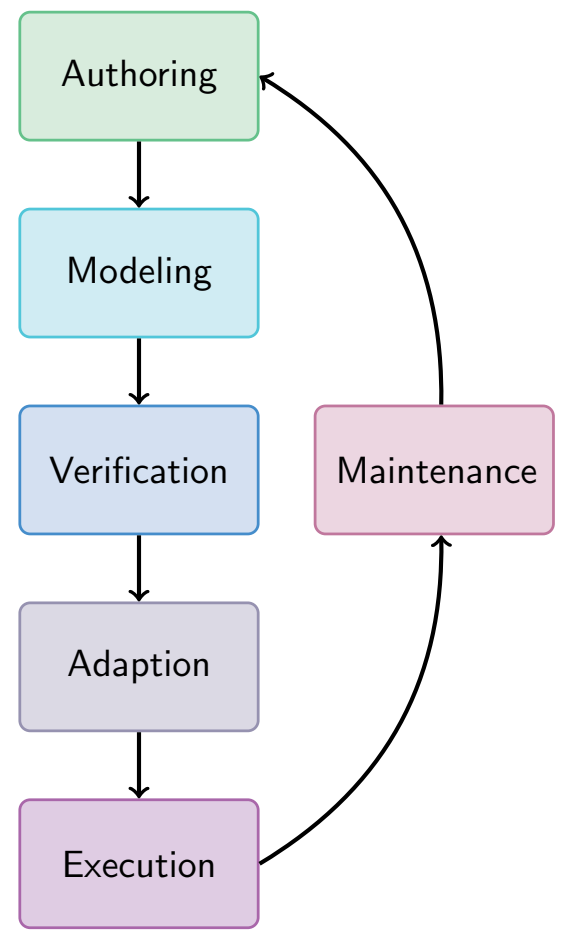

Figure 2.1: Steps of the development cycle of a clinical practice guideline.

From what has been said so far, three main groups of users are involved in the development of a clinical practice guideline:

1. Guideline authors, who create the narrative guideline (e.g., general practitioners, specialists, purchasers of health care services, and patient groups);

2. Guideline modelers, who model, verify, adapt, and maintain the guideline, i.e., knowledge engineers and medical experts;

3. Clinicians, who execute the computerized guideline in clinical practice, i.e., physicians and nurses.

Since we focus on the requirements for a software tool to support the formalization of a guideline and its execution in medical care, we will not deal with the authoring process. We will, however, include another group in our research: the patients. There is evidence in literature that the development of healthcare in recent decades has led to essential changes in the patient's role. Involving patients in decision making has shown to improve the effectiveness of care, to increase the efficiency with which the treatment is delivered, and to lead to a better acceptance as well as to improved outcomes $[4,34,75]$. This is why we focus our research on requirements that pertain to these four user groups: 
1. Guideline modelers,

2. Physicians,

3. Nurses,

4. Patients. 


\subsection{Modelers' Tasks}

The medical knowledge contained in the guideline - originally expressed in natural language text, in tables, or represented in flow charts - has to be translated into a formal language. In order to make the guideline information computer-executable, the information has to be modeled into components that computers can interpret and execute. Thus, a variety of guideline representation languages (guideline representation models) as well as tools to facilitate the translation process have been developed. Each representation language focuses on different aspects. However, "many of the approaches share a hierarchical decomposition of guidelines into networks of component tasks that unfold over time" [67]. Guideline representation languages based on this approach are described as Task-Network Models (TNMs) [19, 46,67].

Different approaches of guideline representation models have been compared by Wang et al. [103], Peleg et al. [67], and de Clercq et al. [21] in order to identify differences and similarities. These articles have outlined the following guideline representation languages which we investigate in detail in the next subsections.

1. Arden Syntax [40],

2. Asbru [79],

3. $\mathrm{EON}[61,96]$,

4. GASTON [20] (the guideline representation model in the GASTON framework),

5. GLIF $[64,66]$,

6. Guide [70],

7. Prestige [39],

8. Prodigy [44],

9. PROforma $[31,32]$,

10. Siegfried [53] (the guideline representation model in the Siegfried system), and

11. Torino [90] (a guideline model developed at the University of Torino).

First let us take a closer look at the different components of these guideline representation languages.

\subsubsection{Components of Guideline Representation Languages}

Since all these approaches use slightly different components to represent the logic of a guideline, and consequently use more or less different terms to refer to guideline plans and components, we will focus on the basic principles constituting a computer-executable guideline representation model. Table 2.1, Table 2.2, and Table 2.3 oppose different terms and components used by diverse guideline representation languages. 
Table 2.1: Different terms used by diverse guideline modeling approaches [103].

\begin{tabular}{|c|c|c|c|c|}
\hline Guideline models & Actions & Decisions & Patient states & Execution states \\
\hline Arden Syntax & Action slot & Logic slot & - & - \\
\hline Asbru & Plan & $\begin{array}{l}\text { Precondition, } \\
\text { Preference }\end{array}$ & Temporal patterns & Plan state \\
\hline EON & $\begin{array}{l}\text { Action, } \\
\text { Consultation- } \\
\text { action }\end{array}$ & Decision & Scenario & -* \\
\hline GASTON & Action & Decision & NA & NA \\
\hline GLIF & Action & Decision & Patient state & $-*$ \\
\hline Guide & $\begin{array}{l}\text { Task, } \\
\text { Wait, } \\
\text { Monitor }\end{array}$ & $\begin{array}{l}\text { Deterministic } \\
\text { decision, } \\
\text { Non-deterministic } \\
\text { decision }\end{array}$ & Implicit in Petri Net & NA \\
\hline Prestige & Protocol & State transition & NA & Procedure state \\
\hline Prodigy & $\begin{array}{l}\text { Action, } \\
\text { Consultation- } \\
\text { action }\end{array}$ & Decision & Scenario & NA \\
\hline PROforma & $\begin{array}{l}\text { Action, } \\
\text { Enquiry }\end{array}$ & Decision & NA & Task state \\
\hline Siegfried & Recommendation & Logic & - & - \\
\hline Torino & $\begin{array}{l}\text { Work action, } \\
\text { Query action }\end{array}$ & Decision action & Conclusion & NA \\
\hline
\end{tabular}

NA: information not available from the publications.

* EON/DHARMA and GLIF have execution states, but they are not in the guideline representation model.

Table 2.2: Different approaches of representing scheduling constrains, subplans of guidelines, and modeling of patient data [103].

\begin{tabular}{llll} 
Guideline models & Scheduling constraints & Subplans & $\begin{array}{l}\text { Modeling of patient } \\
\text { data }\end{array}$ \\
\hline Arden Syntax & Module invocation & - & - \\
\hline Asbru & Plan-body & Plan & - \\
\hline EON & Flowchart & Subguideline & EMR ontology \\
\hline GASTON & Flowchart & Subguideline & Domain ontology \\
\hline GLIF & Flowchart & Subguideline & Domain ontology \\
\hline Guide & Flowchart & Task & Relations \\
\hline Prestige & Protocol composition, & Protocol & Patient record model \\
\hline Prodigy & State transition diagram & & \\
\hline PROforma & State transition diagram & Subguideline & EMR ontology \\
\hline Siegfried & Constraint satisfaction graph & Plan task & Patient data definition \\
\hline Torino & Unidirectional graph & NA & Relations \\
\hline
\end{tabular}

NA: information not available from the publications. 
Table 2.3: Different terms used by diverse guideline modeling approaches $[67,103]$.

\begin{tabular}{|c|c|c|c|c|c|c|}
\hline \multirow[b]{2}{*}{ Model } & \multirow[b]{2}{*}{ Plan } & \multicolumn{5}{|c|}{ Plan component } \\
\hline & & Branching & Action & Decision & Scenario & Subplan \\
\hline Asbru & Plan & Plan type & Plan & $\begin{array}{l}\text { Plan } \\
\text { precondition }\end{array}$ & & Recursive plan \\
\hline \multirow[t]{2}{*}{ EON } & $\begin{array}{l}\text { Management } \\
\text { Guideline }\end{array}$ & $\begin{array}{l}\text { Branch } \\
\text { Synchronization }\end{array}$ & Action & Decision & Scenario & Subguideline step \\
\hline & $\begin{array}{l}\text { Consultation } \\
\text { Guideline }\end{array}$ & $\begin{array}{l}\text { Consultation- } \\
\text { branch }\end{array}$ & $\begin{array}{l}\text { Consultation- } \\
\text { action }\end{array}$ & & & $\begin{array}{l}\text { Consultation } \\
\text { guideline part of } \\
\text { scenario }\end{array}$ \\
\hline GLIF & $\begin{array}{l}\text { Guideline, } \\
\text { Macro }\end{array}$ & $\begin{array}{l}\text { Branch } \\
\text { Synchronization }\end{array}$ & Action & Decision & Patient-state & $\begin{array}{l}\text { Guideline or Macro } \\
\text { called in Action or } \\
\text { Decision steps }\end{array}$ \\
\hline Guide & Guideline & $\begin{array}{l}\text { Synch-\&, } \\
\text { Synch-Or }\end{array}$ & $\begin{array}{l}\text { Task, } \\
\text { Wait, } \\
\text { Monitor }\end{array}$ & $\begin{array}{l}\text { Deterministic } \\
\text { decision, } \\
\text { non- } \\
\text { deterministic } \\
\text { decision }\end{array}$ & & $\begin{array}{l}\text { Any task can be de- } \\
\text { composed }\end{array}$ \\
\hline \multirow[t]{2}{*}{ Prodigy } & $\begin{array}{l}\text { Decision/ } \\
\text { Manage- } \\
\text { ment map }\end{array}$ & & Action & & Scenario & $\begin{array}{l}\text { Subguideline Step } \\
\text { or called in Action } \\
\text { step }\end{array}$ \\
\hline & $\begin{array}{l}\text { Consultation } \\
\text { Template }\end{array}$ & $\begin{array}{l}\text { Consultation- } \\
\text { branch }\end{array}$ & $\begin{array}{l}\text { Consultation- } \\
\text { action }\end{array}$ & & & $\begin{array}{l}\text { Consultation tem- } \\
\text { plate part of sce- } \\
\text { nario }\end{array}$ \\
\hline PROforma & Plan & $\begin{array}{l}\text { Action, Enquiry, } \\
\text { Decision }\end{array}$ & $\begin{array}{l}\text { Action, } \\
\text { Enquiry }\end{array}$ & Decision & & Plan task \\
\hline
\end{tabular}

To get a more detailed understanding of the tasks and the resulting information needs involved in generating a formal model of a clinical guideline, we will outline different formalization tools and how to use them in the following subsections.

\subsubsection{Formalization Process}

Clinical practice guidelines are narrative documents with challenging characteristics such as lacking regularity of the used vocabulary; for instance, concept definitions are sometimes ambiguous. Additionally, background knowledge is not stated explicitly, and the writing style is extremely variable. Thus, human analysis is required to create a computer-interpretable model [73]. Due to the complexity of translating a narrative clinical practice guideline into a formal guideline representation model, a variety of methods and tools have been developed to support this task. Table 2.4 opposes the different guideline representation languages to tools used to obtain a corresponding formalized model of the original guideline.

\section{Model-Centric Formalization}

Most guideline formalization methods are so called model-centric approaches. Initially, a medical domain expert formulates a conceptual model of a guideline, based on the original narrative document but without a direct relationship between the original text and the model. Only selected parts of the guideline text are linked to this model for the sake of documentation. Thus, it is the interpreting expert who bears the responsibility for the validity of the conceptual model. This often flowchart-based model is then converted into a fully computer-executable represen- 
Table 2.4: Different guideline representation languages opposed to corresponding guideline formalization tools [47].

\begin{tabular}{lll} 
Model & Model-Centric Tools & Document-Centric Tools \\
\hline Asbru & AsbruView & DELT/A, Stepper, Uruz (Degel), PBW (Degel) \\
\hline EON & Protégé & - \\
\hline GASTON & Weaning Guideline Editor & - \\
\hline GEM & - & GEM Cutter \\
\hline GLIF & Protégé & - \\
\hline Guide & Guide Editor & - \\
\hline Prestige & GAUDI, GLEAM & - \\
\hline Prodigy & Protégé & - \\
\hline PROforma & AREZZO, TALLIS, Protégé & -
\end{tabular}

tation. Hence, the model-centric approach of formalizing a clinical guideline is a top-down process $[72,86]$. Examples of model-centric tools supporting the formalization of clinical guidelines are AsbruView [51], which is designed to model Asbru plans; AREZZO [42] and TALLIS [84], which support the modeling process into PROforma; and Protégé ${ }^{1}$ [33], which supports the transition of guidelines into the representation languages EON, GLIF, and PROforma [48].

The following subsections contain an outline of the modeling process of a clinical guideline within the Standards-Based Sharable Active Guideline Environment (SAGE) project [6] as well as of the modeling process using the Guidelines Interchange Format (GLIF) [64,66].

SAGE. The Standards-Based Sharable Active Guideline Environment (SAGE) project [6] was aimed at developing a standards-based, comprehensive technology infrastructure that enables medical experts (with informatics training) to author, encode, and disseminate interoperable, electronic clinical practice guidelines. Moreover, the project should enable health care organizations to deploy those guidelines easily within any standards-conforming clinical information system [93]. In context of this project Tu et al. formulated different steps necessary to model a clinical practice guideline by means of this model-centric approach (see Figure 2.2) [97]:

1. Identification of usage scenarios of guideline-based care in clinical workflow: In a first step clinicians need to identify clinical scenarios that are detailed enough to support the integration of executable guideline knowledge into clinical workflow.

2. Distillation and disambiguation of guideline knowledge relevant to these usage scenarios: In a second step clinicians with informatics training need to analyze the desired guideline recommendations and determine the knowledge and logic needed to generate these recommendations. This knowledge is derived from guideline texts, medical literature, and the expertise of the clinicians. For instance, the term "contraindication to Hep B" is disambiguated by the definition "anaphylactic reaction to hepatitis B vaccine".

\footnotetext{
${ }^{1}$ Protégé is a free, open source ontology editor and knowledge-base framework. The extensible development environment allows for ontology development and knowledge acquisition in order to facilitate the authoring of clinical guidelines in various guideline representation languages (e.g. EON, GLIF, Prodigy, and PROforma).
} 


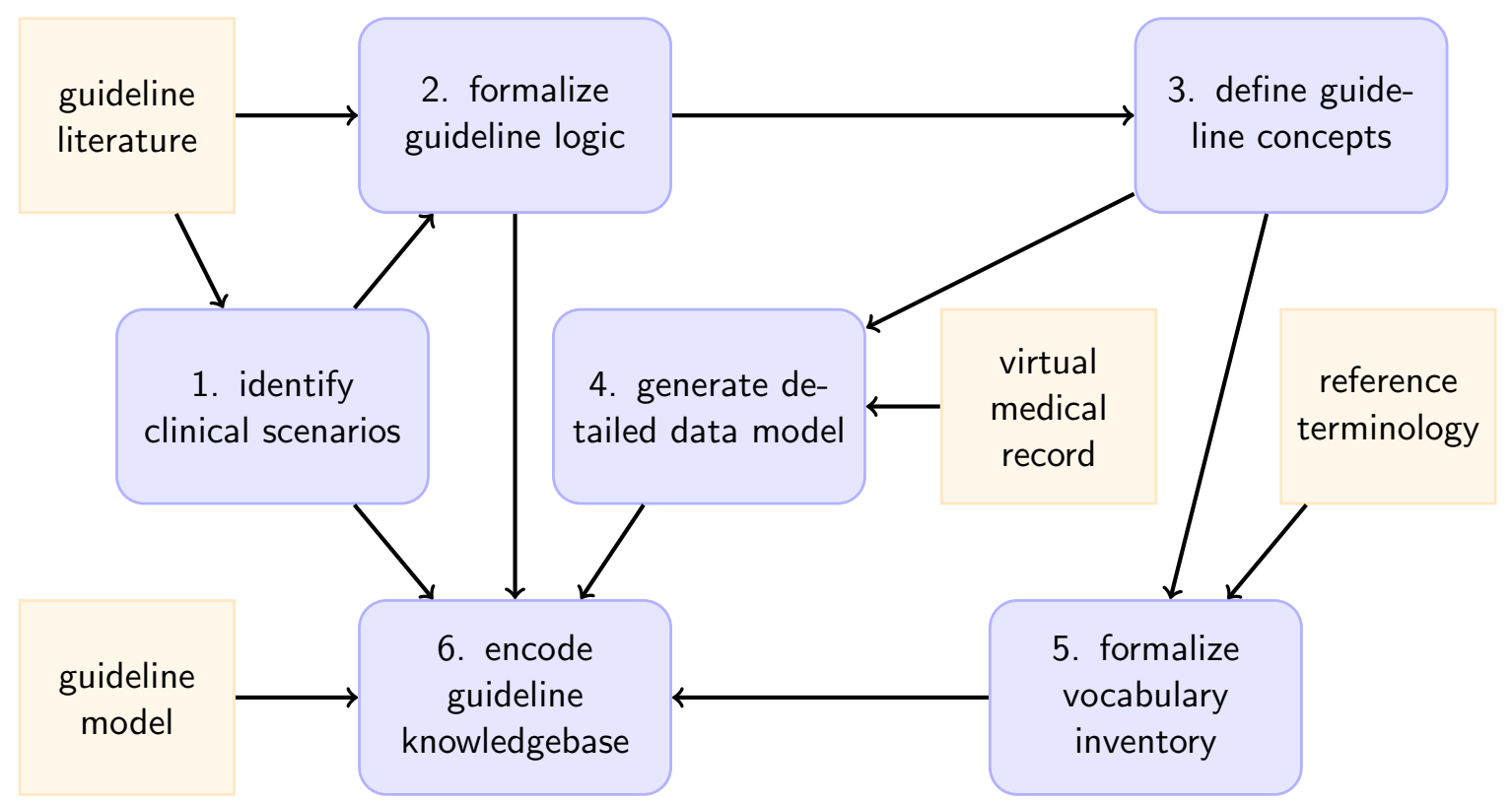

Figure 2.2: SAGE: Steps in modeling clinical practice guidelines for integration into workflow [97].

3. Identification of clinical concepts: Now, clinical concepts used in the distilled guideline logic are to be identified, extracted, and recorded (e.g. "hepatitis B vaccine" and "anaphylactic reaction").

4. Creation of a detailed data model: The previously identified concepts are instantiated as detailed data models with respect to the constraints on classes of a "virtual medical record" $(\mathrm{vMR})^{2}$ [43]. For instance, allergy information is modeled as an instance of the class Allergy which has the attributes code, allergen, reaction, and effective time. Hence, "Anaphylactic reaction to hepatitis B vaccine" is modeled as Allergy where "vaccines allergy" is assigned to the code attribute, allergen is defined as "hepatitis B vaccine", and reaction is "anaphylaxis".

5. Creation of a standard vocabulary: Terms used in a guideline knowledge base need to be mapped to corresponding terms in the electronic patient record of the particular institution where the guideline is to be implemented.

6. Modeling usage scenarios and guideline knowledge: Care processes are modeled by means of activity graphs and recommendation-sets. A recommendation set is related recommendations that are applicable in one or more shared contexts. Activity graphs illustrate these recommendation sets in a flowchart-like representation. The different shapes represent different node-functions [97]:

- Context Nodes: used to represent clinical settings (e.g. outpatient encounter in a general internal medicine clinic), care providers involved in the recommendation process, required clinical resources, important patient states (e.g. patient age), and potentially triggering events (e.g. a patient's checks-in to the clinic);

\footnotetext{
${ }^{2} \mathrm{~A}$ vMR is a simplified view of a patient medical record that represents only classes relevant for decisionsupport.
} 
- Decision Nodes: used to represent decisions in the recommendation process (e.g. a Boolean precondition for an action);

- Action Nodes: used to represent a set of actions that should be performed either by a computer system or by a healthcare provider;

- Routing Nodes: used for branching and synchronization of multiple concurrent processes.

These nodes are then connected by arrows to form a network representing the clinical algorithm (see Figure 2.3). To create these activity graphs, they use the Protégé tool [33].

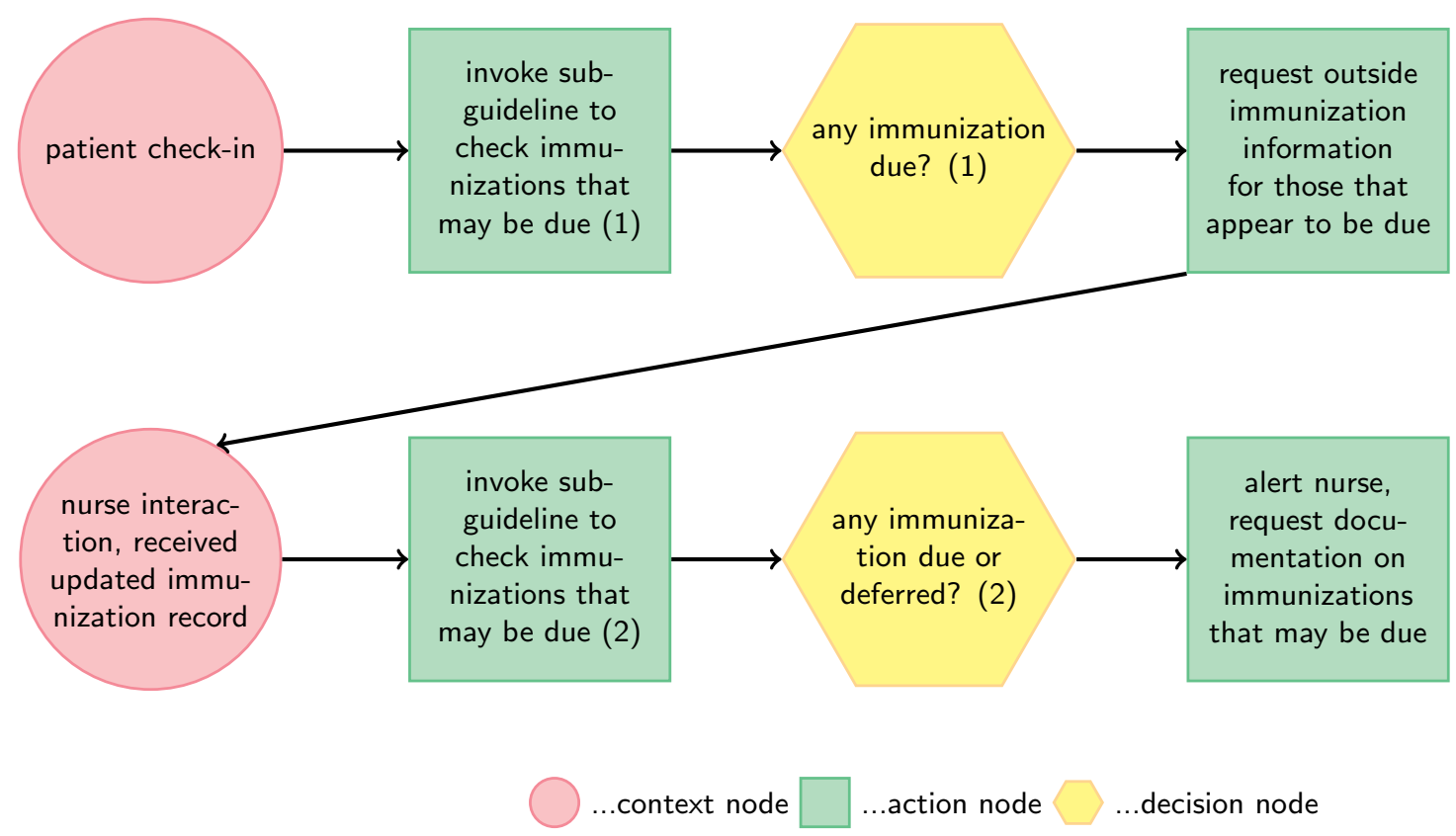

Figure 2.3: Part of an activity graph as encoded in the SAGE Guideline Model [97] (reproduction of the activity graph originally generated by means of the Protégé tool).

GLIF. The Guidelines Interchange Format (GLIF) of the Intermed Collaboratory is a guideline representation model which is focused on a detailed specification of guideline recommendations. GLIF guideline models are usually represented in a flowchart-like way. The nodes of the graph are guidelines steps, including: action steps, decision steps, branches, synchronization steps, and patient state steps. Moreover, criteria of condition for each decision option are specified (e.g., strict-in criteria, strict-out criteria, rule-in criteria, and rule-out criteria) [10]. Buchtela et al. formulated different steps necessary to create a GLIF model of a clinical practice guideline [10]:

1. Generating a flowchart-like GLIF model corresponding with the textual guideline: specifying a logical and process structure of the guideline, all fundamental parameters and their interrelationships.

2. Encoding the graph in the XML format.

3. Creating a list of basic parameters (e.g., measurable values) and derived parameters (obtained in a arithmetical or logical operation above basic parameters). 
However, literature provides more detailed descriptions on how to model clinical guidelines in a document-centric way. The following subsections deal with formalization processes with different formalization tools, as well as a sophisticated approach of step-wise formalization by means of information extraction.

\section{Document Centric Formalization}

Another approach to formalize a narrative guideline document is called document-centric. The original text of the document serves as a starting point which is systematically marked up, whereupon the marked-up text parts are filled into knowledge containers. Thus, the text is structured into a - usually XML-based - semiformal model. Often multiple steps and consequently multiple intermediate states are necessary to create an operational model. Because of this, the guideline model is built bottom-up. In contrast to the model-centric approach, the final model is closely bound to the literal text of the original guideline [72,86]. Examples of document-centric tools supporting the formalization process are the GEM Cutter [68] - one of the first tools utilizing a document-centric approach - which is tailored to transform guidelines into the GEM format; Stepper [72,86]; the Document Exploration and Linking Tool / Addons (DELT/A) [101]; and finally there is Uruz which is part of the Digital Electronic Guideline Library (Degel) framework [80].

Some of these tools come with detailed descriptions of how to use them to create a guideline representation model.

GEM Cutter. The GEM Cutter II [60] (the successor of the GEM Cutter [68]) is an XML editor to facilitate the mark-up of the narrative text of a CPG into the GEM format [81] (see Figure 2.5). It was developed by the Yale Center for Medical Informatics at Yale University School of Medicine. The user interface of the GEM Cutter II (see Figure 2.4) consists of three vertical panes, whereas the text of the guideline document is displayed in the left pane; in the middle pane, a tree view of the GEM hierarchy (see Figure 2.5) is displayed, and the right pane allows for text editing and provides additional important information about the GEM elements. According to Shiffman et al. the following steps are required to translate the knowledge contained in a CPG into a computer-executable format and to integrate the information into clinical workflow [82]:

1. Selection of a guideline and specific recommendations for implementation: If several guidelines exist on the same topic, a specific practice guideline must be chosen. Therefore, the quality of the guideline as well as its likely implementability have to be considered. Within a second selection step the specific recommendations to be implemented have to be chosen.

2. Markup of the guideline text: Specific guideline knowledge components such as recommendations, definitions, and algorithm hierarchies have to be identified in the text and marked up by means of XML tags. Additional XML elements describing the guideline's purpose, intended audience, target population, and schemes for rating evidence quality as well as recommendation strength are recommended. The GEM cutter facilitates the markup of the guideline text.

3. Atomization: Single concepts of the narrative recommendation's text have to be extracted and refined by "removing unnecessary words, changing verb phrases from passive to active, reducing decision variables to prototypic nouns with descriptors occupying the 


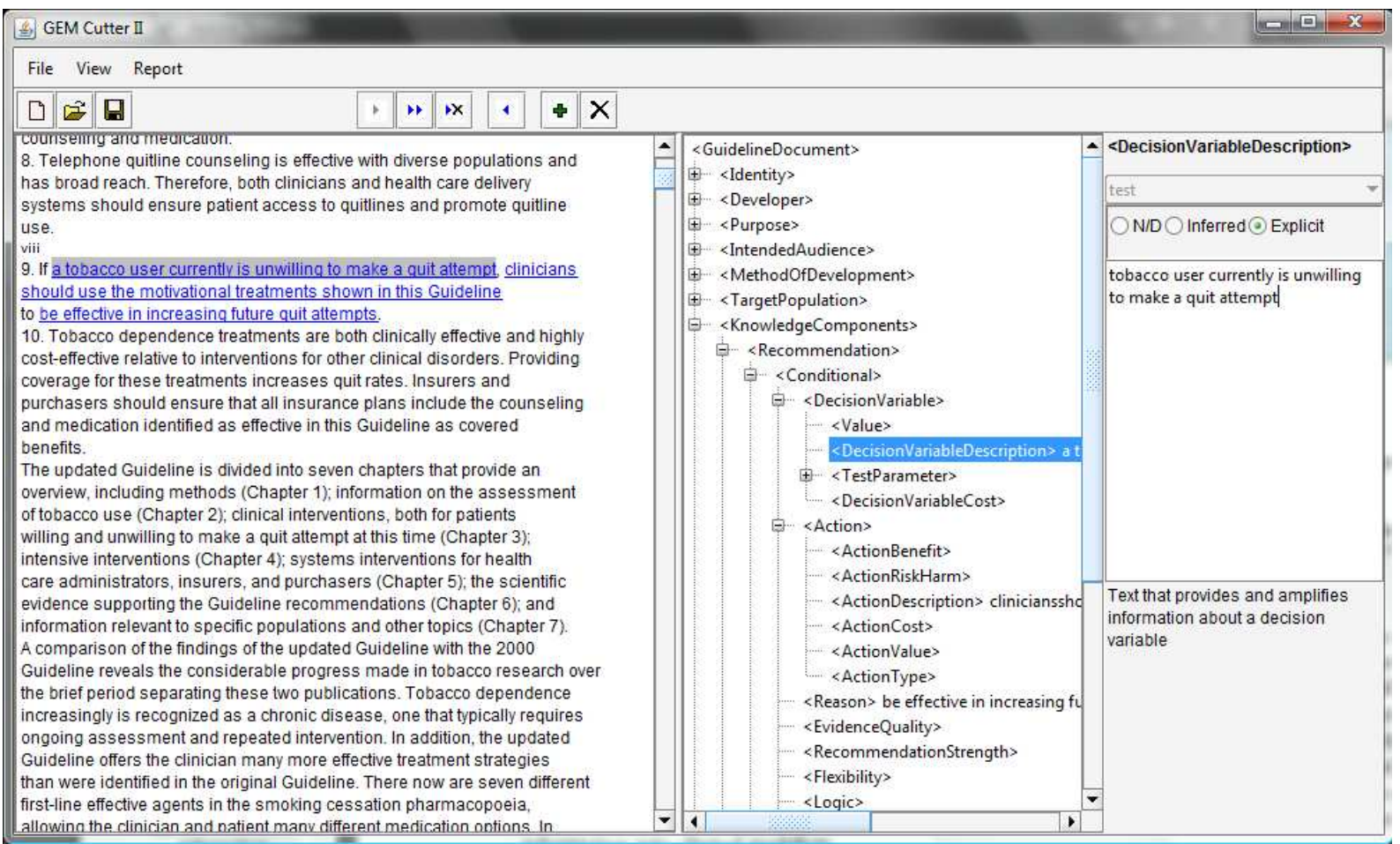

Figure 2.4: Screenshot of the GEM Cutter II editor.

〈value element, and stating actions and directives as verbs in active voice with associated direct and indirect objects and modifiers." [82]

4. Deabstraction of recommendation concepts: The deabstraction of recommendations is aimed at adjusting the level of generality at which an action or a decision variable is described in order to allow for operationalization. For instance, the guideline text

"If the patient's asthma is not optimally controlled with the initial therapy, and medications are used correctly, additional step-3 therapy is recommended." [82]

needs to be deabstracted. The term "medications are used correctly" is an abstract concept that may be difficult to formalize. It implies that the patient adheres to the prescribed medication and uses the right inhalation technique. Right inhalation technique, in turn, means that the patient uses a spacer, takes slow inhalations, and repeats the inhalation after 1-4 minutes.

5. Disambiguation of recommendation concepts: To disambiguate recommendations requires providing a single semantic interpretation for a recommendation statement. Ambiguity is valid only when values of decision variables are not mutually exclusive. For instance:

"The NHLBI guideline provides a table for classification of asthma severity that includes criteria based primarily on symptom frequency. The proposed classifier also includes an ambiguous set of descriptors for 'asthma exacerbation' that are poorly defined and not mutually exclusive. Analysis of these descriptors showed that they addressed 3 different dimensions: frequency, severity, and duration of exacerbations. For example, duration was described as 'frequent' in one instance 


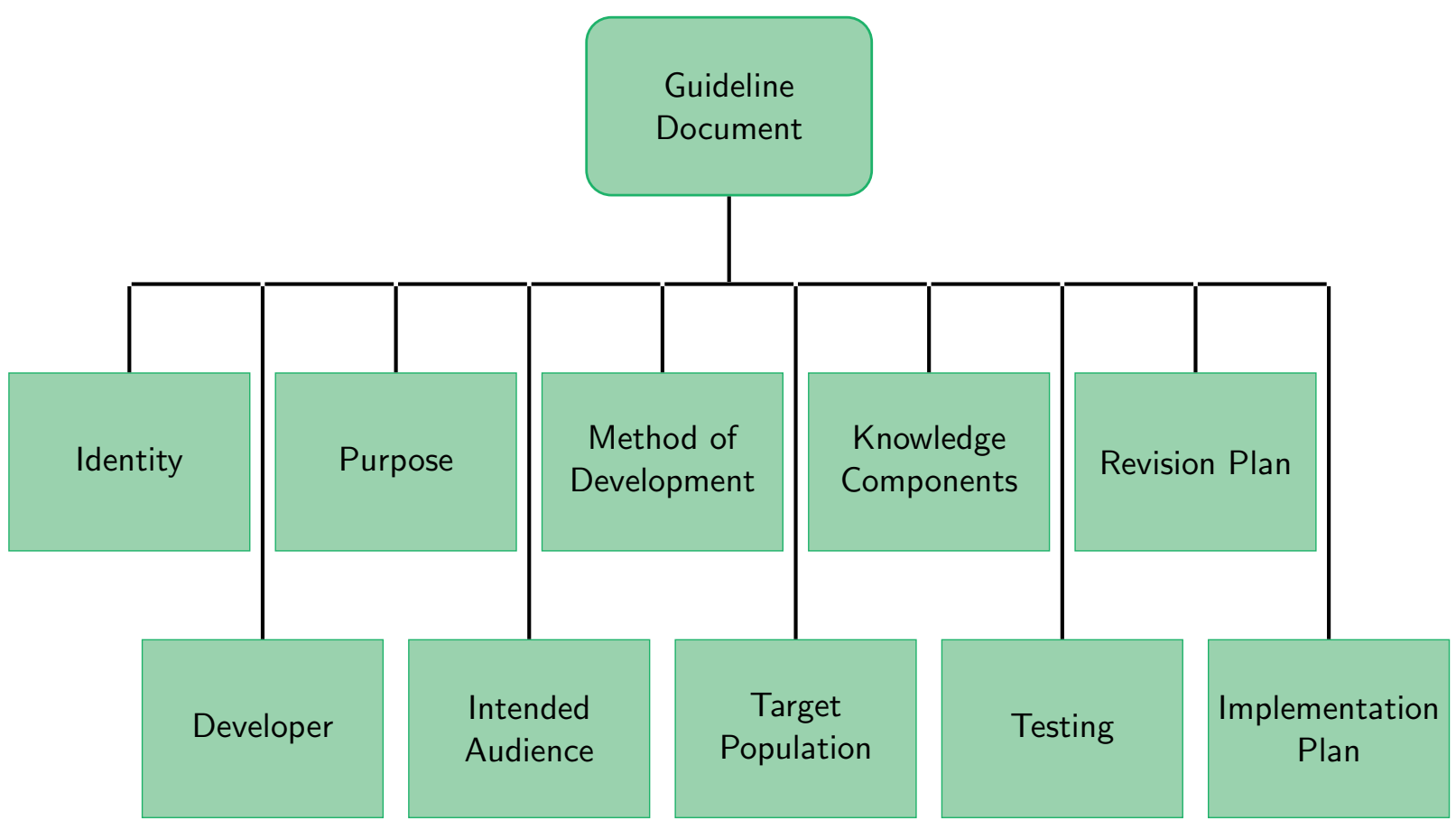

Figure 2.5: GEM: top level elements [60].

and its alternative was ' $\geq 2$ times per week.' Severity was described using three nonexclusive values: 'may affect activity', 'affect(s) activity', and 'intensity may vary.' Finally, duration could take on values of 'may last days' or 'brief (from a few hours to a few days).' The semantic overlap inherent in these descriptors for exacerbations indicated that 'exacerbations' would not be useful for classification of asthma severity." [82]

6. Verification of rule set completeness: Completeness verification is crucial to assure that each recommendation provides guidance in all situations that a clinician may face, i.e., all logically possible combinations of condition states need to be addressed by the guideline model. A guideline model not describing appropriate actions in all situations will lead to potentially avoidable practice variation.

"The patient's response to therapy should be monitored carefully. When benefits are sustained, a step down in therapy should be attempted. If there are no clear benefits, treatment should be stopped, and alternative therapies or diagnoses should be considered." [82]

The guideline gives recommendations for the cases "sustained benefits" and "no clear benefits", which are potential alternative values for the decision variable "benefits". In case the benefits are present but temporary, the guideline fails to provide recommendations.

7. Addition of explanations: Describing the reasoning behind recommendations, for instance by extracting text directly from the guideline document, has been considered important for clinical decision support [89]. The GEM standard provides the reason element to justify the proposed service. In addition, information categorized in the objective, ratio- 
nale, decision variable description, test parameter, action description, action benefit, and risk-harm elements can be useful.

8. Building executable statements: Now, the atomized, deabstracted, and disambiguated actions and decision variables need to be rearranged into logical statements that are better suited for translation into computer-executable statements. Guideline recommendations can be expressed in statement logic by using a limited number of logical operators (conjunction, disjunction, negation, conditional, and parentheses for grouping) [54]. This elementary set of operators has proven sufficient in most cases. For the encoding of more complex relationships, such as temporal relationships between individual statements, more sophisticated notations are needed.

9. Specification of origins of decision variables and insertions of recommended actions: A critical step for workflow integration is the indication of a source or origin in the clinical environment for each decision variable as well as of an insertion point in the care process for each recommended action.

10. Definition of action types and selection of associated beneficial services: Shiffman et al. have empirically defined an action palette, i.e., a limited set of predefined action types that are suited to categorize all activities recommended in a large number of randomly selected guidelines [28]. This palette is made up of four major categories: gathering information, interpreting information, performing a task, and arranging for or organizing additional care. This step is aimed at facilitating the formalization process.

11. Choice of interface components: Interface components must be selected and arranged. Screen mockups can be used to facilitate the interface design process of the information system. The adherence to principles of effective interface design as well as the involvement of users is critical to create an optimal user interface.

12. Creation of requirement specification: In context of the above process, a requirement specification is to be generated which serves as a starting point for an iterative development process. "One or more use cases may be used to document functionality of each guideline recommendation that is to be implemented. A high-level structural view of the static concepts and concept attributes that define the structure of the guideline domain and an activity diagram that depicts the passing of information among stakeholders may be useful for implementers who are familiar with the Unified Modeling Language (UML) [30]. Finally, a glossary that defines critical concepts is valuable." [82]

This description of modeling a CPG is specifically tailored towards the formalization process by means of the GEM Cutter. Other tools and techniques are outlined in the following. Subsequently, we have normalized the findings of these approaches in Section 3.1.

Stepper. Stepper $[72,86]$ was developed by the EuroMISE centrum - Kardio and the University of Economics, Prague, Czech Republic. It is a mark-up tool to transform narrative guidelines step-by-step into formal models. Stepper decomposes the guideline formalization process into an unrestricted number of user-definable steps in order to facilitate the conversion into a formal guideline representation. In 2003 [86] Svátek and Růžička suggested six levels of formalization, where each level is represented by a specialized XML-based language together with its DTD (Document Type Definition). 
1. Input text format: The original document is converted into the favored input format XHTML - the XML version of HTML.

2. Coarse-grained semantic mark-up: Large coherent text chunks of the guideline are semantically marked-up to be basic elements, for instance causal relation, concept definition, goal, procedural fragment, and other information (in accordance with the example shown in Figure 2.6). The corresponding text chunk of the document is marked up and subsequently the category is chosen by pressing one of the buttons in the upper-right pane. In addition, irrelevant parts of the document are removed. This step can be done by a knowledge engineer with limited medical background knowledge [72].

3. Fine-grained semantic mark-up: The previously classified text chunks are refined into sub-elements, which are arranged in a tree structure. For instance, a procedural fragment can be transformed into a structure of scenarios. Medical experts need to add required background knowledge and to reformulate some phrases or terms in order to keep consistency. Important clinical parameters occurring in the guideline are characterized by means of a data dictionary.

4. Universal knowledge base: The structure of the document is rearranged as to achieve modularity, and cross-references in the text are constituted. Thus, the original document is converted into a universal knowledge base. This involves the collaboration of knowledge engineers and medical experts.

5. Export-specific knowledge base: The structure of derived elements is adapted with respect to the target representation. An export-specific knowledge base is created from the universal one.

6. Target computational representation: In the last processing step, the export-specific knowledge base is transformed into the target computer-executable format, which can be done fully automatically by using XSL style sheets.

According to Svátek and Růžička there are six major general tasks evolving from the formalization of a clinical practice guideline [86]:

1. Replacement of generic linguistic expressions: The structure of generic linguistic expressions such as causalities, decisions, or definitions (e.g., "if...", "but...", "should be...", "is always...", etc.) have to be identified in the text and then replaced by standardized formal structures.

2. Removal of useless text: Irrelevant text parts of the guideline document have to be removed.

3. Modularization of knowledge elements: Coherent knowledge elements within the guideline have to be modularized, whereby they are made independent from the surrounding context in the text.

4. Shift to controlled vocabularies: In order to achieve consistency, diverse free-text terms referring to the same domain concept have to be identified and consequently replaced by standardized terms.

5. Addition of implicit knowledge: Implicit knowledge or required background knowledge has to be added. 


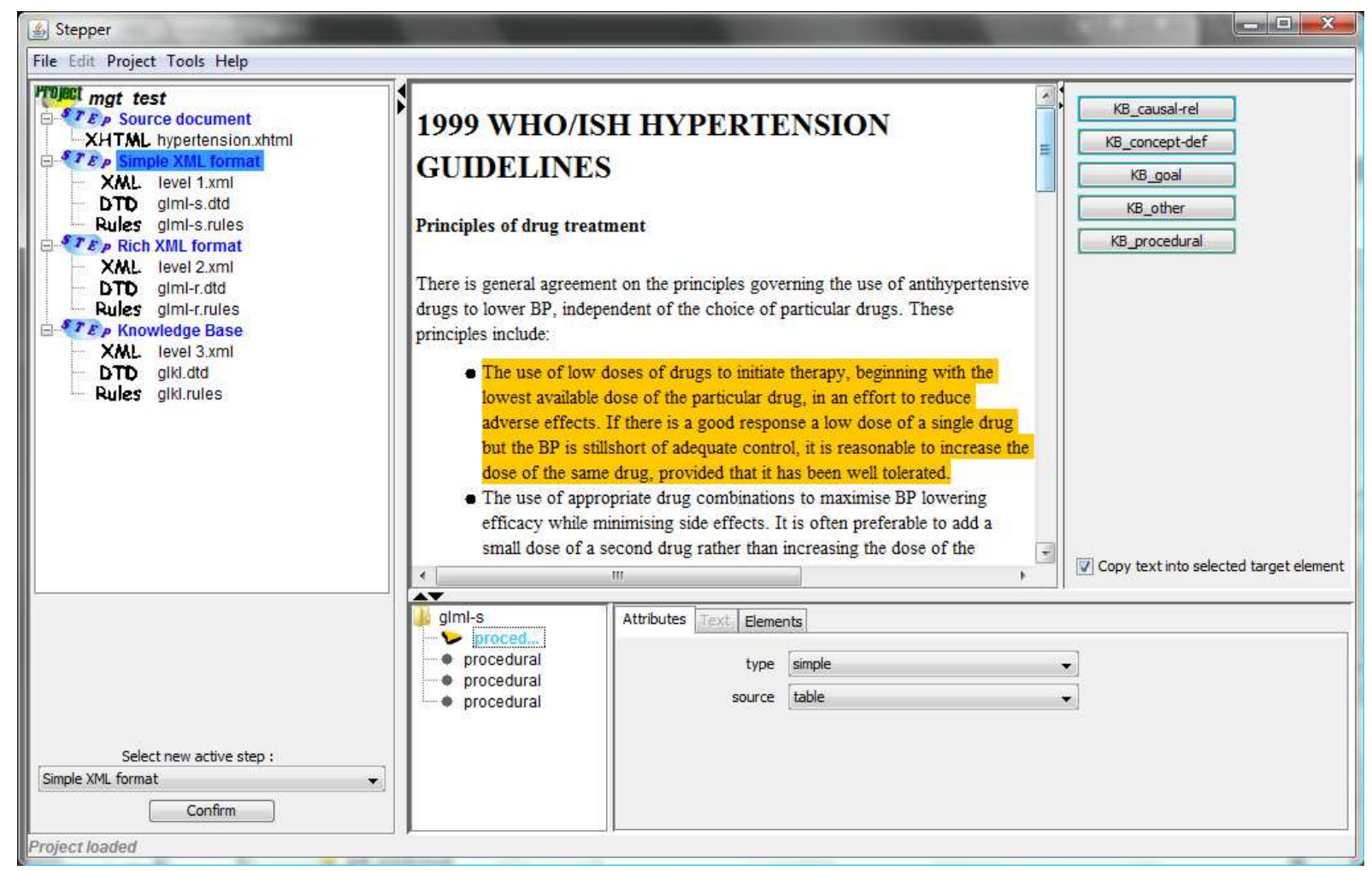

Figure 2.6: Stepper: step-wise guideline formalization.

6. Handling of vague formulations: Loose formulations regarding the state of measurable parameters, such as "bad response to therapy", "frequently", or "several times a week" have to be assessed and replaced by concrete and appropriate values.

DELT/A. The Document Exploration and Linking Tool / Addons (DELT/A), which is based on the Guideline Markup Tool (GMT) [101], facilitates the translation of free text guidelines into a formal representation by providing two main features: it provides

- Means to define and maintain links between the textual guideline and its formal representations, which allows the user to find out where a certain value of the formal model comes from, and

- Macros for frequently used design-patterns of specific XML languages (e.g., Asbru [79] and MHB [78]).

DELT/A supports a multi-step transformation process of clinical guidelines. It produces intermediate representations of the document for each formalization step until the target representation is reached. The graphical user interface of DELT/A is composed of three panes. The top left pane shows the source file - the textual clinical guideline (in HTML format) or an intermediate representation of the guideline (in XML format); the top right pane displays the currently generated equivalent formal representation in the target XML-language notation of the document in the left pane, and the bottom pane provides a structured view of available macros as well as a preview of the currently selected macro (see Figure 2.7). A two-step transformation process is described as follows [100]: 
1. The user opens the original guideline document in the left pane, creates an empty XML file of the intermediate XML language (e.g., MHB) in the right pane, and chooses an appropriate macro file in the lower left pane.

2. To create the intermediate XML representation, the user selects a text chunk in the original document as well as the XML element in the right pane where the element is to be inserted. Then the user chooses the appropriate macro from the lower left pane, whereupon a preview of the chosen macro is displayed in the lower right pane. On clicking a button the newly created XML element is inserted into the XML structure in the upper right pane.

3. This procedure is repeated until the relevant knowledge of the guideline is modeled in the intermediate XML document.

In a second turn, the intermediate representation is further formalized into the target XML format:

4. The intermediate XML file is swapped to the left side. Again the user has to create a new empty file of the target XML language in the right pane and choose the corresponding macro file.

5. The user continues to mark-up text chunks in the document at the left and to insert XML elements into the target XML structure at the right by applying macros.

One possible intermediate representation format that can be generated by means of the DELT/A editor is the MHB format which is described in detail in the following subsection.

MHB. The Many-Headed Bridge (MHB) format by Seyfang et al. [78] is an XML language aimed at representing an abstraction state of a clinical guideline while translating it into the target representation language. It was designed to close the large gap between the natural language text and the formal representation of a clinical guideline, thus facilitating the formalization process. MHB provides chunks which correspond to a certain bit of information in the natural language text (e.g., a sentence, part of a sentence, or more than one sentence). These chunks are internally structured into aspects which are grouped by the following dimensions: control flow, data flow, temporal aspects, evidence, background information, resources, patient related aspects, and document structure [78].

Control Flow. It specifies the execution order of tasks, their decomposition, and the gathering of information; it covers the following aspects:

- Two MHB elements are used to specify decisions: if-then elements and option group elements. If-then elements contain a pair of conditions and results along with modifiers (condition-modifier and result-modifier). Option group elements are used to group related decisions.

- The ordering and decomposition of tasks is specified by the MHB element decomposition, which defines the decomposition of tasks into subtasks and constraints on their execution order relative to each other.

- When several tasks are performed independently (e.g. in parallel), the synchronization element defines a task or a number of tasks which must be completed before the next task is processed. 


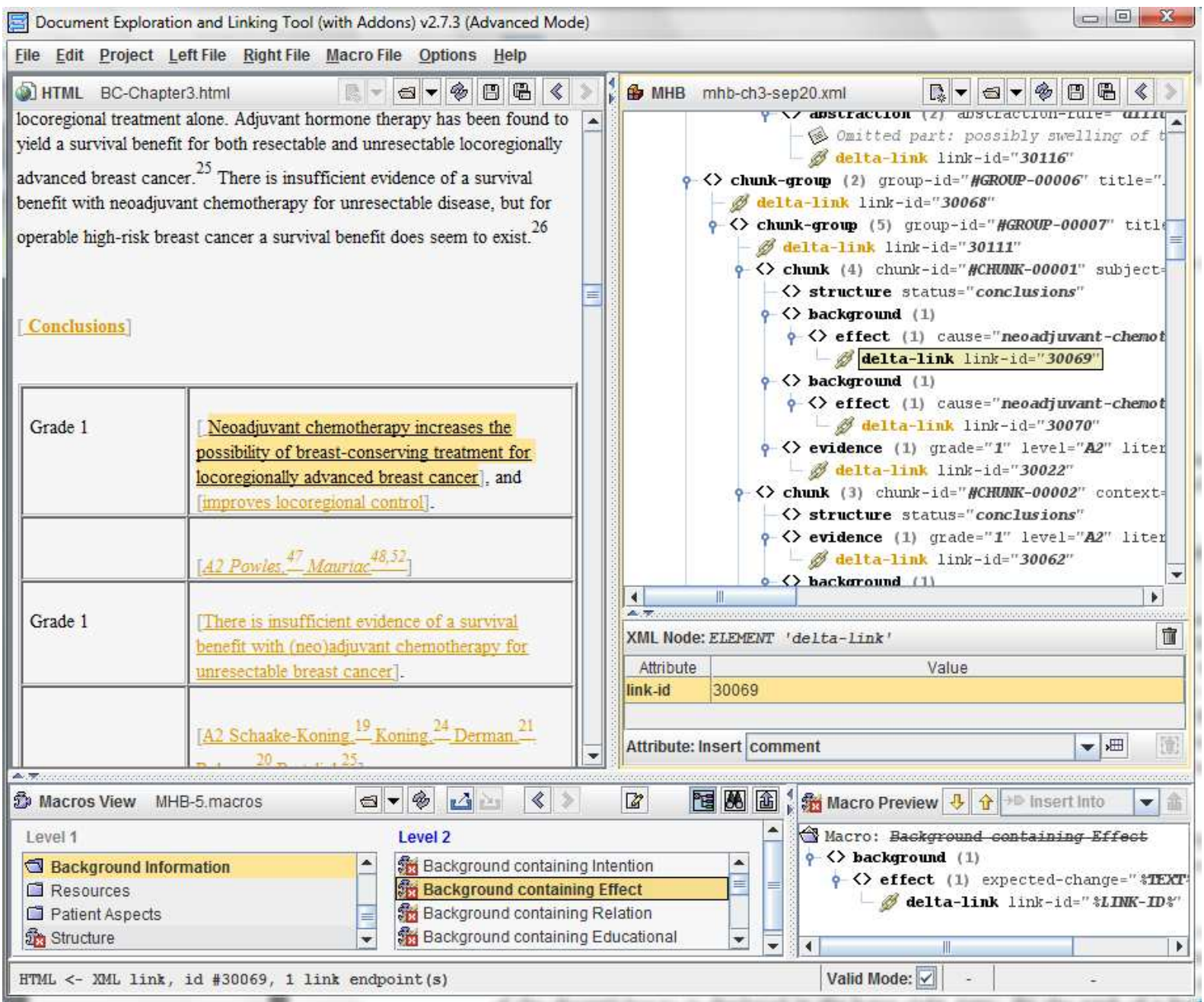

Figure 2.7: DELT/A - The two top panes show linked views of the same guideline. The top left view shows the textual guideline while the top right view shows its formal representation. The bottom pane is the macro view which provides macros to facilitate the creation of the formal guideline representation [101].

- Repetition defines if a task is performed more than once; if so, how often or at which times during another task, and

- Atomic actions, i.e., activities or tasks which are described in a sentence without further details, are defined by the MHB element clinical-activity.

Data Flow. It describes the data processing involved in the diagnosis and treatment. For the formal version of a guideline, the definition of a data item is necessary. Thus, the MHB element data contains the following child-elements:

- The definition consists of a name, a type, and often a range of plausible values and preferred unit.

- Usage holds the variable or piece of information which is used in this MHB element,

- Into the input element, the user can insert a certain bit of information, and 
- Abstraction rules which indicate the calculation or abstraction of the data item based on others.

Temporal aspects. MHB elements may have qualitative or quantitative temporal aspects. MHB can be used to model the complex Asbru time-annotations. For each quantitative temporal dimension, i.e. start, end, and duration, MHB allows for defining the minimum, maximum, estimate, and precise value. Even more, MHB allows for modeling qualitative temporal relations (e.g. "A is started after the end of B").

Evidence. Evidence for a statement within a guideline can be given in explicit and implicit form. Explicit references, i.e. summary statement of the evidence or literature references, can be modeled by means of the (optional) attributes grade, level, importance, and literature-reference. Implicit references in the guideline can be made explicit in the MHB model with the attribute is-based-on, which holds a reference to another MHB element.

Background Information. To model the background information given in the guideline this can be information aimed at motivating the reader to follow the guideline or information to complement the statements in the recommendation part - MHB provides the following elements:

- Intentions of described actions and recommendations inform and motivate the user. In the Protocure project [5] they provide important input to the proof process.

- Effects are relations between data or actions and phenomena or events so that one is the cause of the other.

- Relations are similar to effects but do not imply a cause and effect relation.

- Educational information can be given to inform the physician or the patient and to allow for discussing the recommendations in detail; for instance, some statements of the guideline may not instruct an action or any activity, but still contain important information.

- Explanations provide information directly related to explain the actions resulting from following the guideline.

- Indicators are measures to define to which degree a guideline is followed in practice, or the success of following the guideline. Indicators can be derived from key recommendations of a guideline. They are not yet part of guidelines (at CBO [92]) but will be integrated in the future, with respect to AGREE item 21 [91]: "The guideline presents key review criteria for monitoring and/or audit purposes".

Resources. For each action, resources of various nature are needed: personnel resources (e.g. working time of clinical staff), devices (e.g. treatment facilities), and financial costs (e.g. drug costs). This MHB element is intended to give this information independently of the information on the above items.

Patient Related Aspects. Several general issues mentioned in a guideline refer to the patient's perspective of treatment. Risk (connected to certain treatment options or diagnostic actions), patient discomfort (e.g. side effects of treatments), and health prospective are means to encode these aspects. 
Document Structure. The status of a statement in the guideline - whether it was found in a narrative section or in a distinct recommendation - forms an important context of its interpretation. This information helps in the interpretation of the intermediate representation and in the further formalization process.

In addition to the formalization with these specific tools, Kaiser et al. introduced a documentcentric approach of formalizing a CPG, using semi-automatic, step-wise information extraction methods.

Semi-Automatic Step-Wise Information Extraction Methods. Kaiser et al. developed several heuristics (called LASSIE: modeLing treAtment proceSSes using Information Extraction) to support the guideline-modeler by automating parts of the modeling process. Moreover, they give a detailed description of the single formalization-steps to make the modeling process traceable and comprehensible. The single steps of this approach are [46]:

1. Detection and exclusion of irrelevant text parts.

2. Detection of relevant sentences.

3. Generation of an intermediate representation listing all relevant sentences linked to the marked-up guideline document.

4. Structuring of relevant sentences by assigning them to hierarchical groups.

5. Extracting required information: therapy instruments (i.e., surgical procedures), dosage information (drug administration), duration of the therapy, iteration information, and conditions which have to be fulfilled to perform an action.

6. Categorizing sentences in actions or negative actions (i.e., instructions that an action should not be performed).

7. Identifying annotations and assigning them to actions or negative actions. Annotations always belong to at least one action or negative action.

8. Grouping actions into selections, where one action of each selection has to be selected to be executed.

9. Modeling actions. To do so, the following parameters are to be defined: the assigned annotation sentences, treatment instruments and their $M e S H^{3}$ ids, dosage, duration, or iteration of drug administration, conditions, as well as the selection id - in case the action is part of a selection.

10. Detecting relations between actions - explicitly mentioned within the text or implicitly given by the document structure.

11. Modeling relations. To do so, the following parameters are to be defined: the source action and the destination action, as well as the relation type, for instance: succeeding, proceeding, overlapping, etc.

\footnotetext{
${ }^{3}$ Kaiser et al. used medical terms based on a subset of the Medical Subject Headings (MeSH) [99] of the United States National Library of Medicine.
} 
12. Generating an intermediate representation containing actions and their relations as well as the hierarchy and nesting of groups; each information has to be linked to the original source data (links are inherited from the last intermediate representation).

13. Transformation into target representation.

The formalization processes described above use different techniques, emphasize different aspects, and were proposed by different institutions. We give an outline of normalized findings of these approaches in Section 3.1. 


\subsection{Clinical Information}

After investigating the different tasks of formalizing a clinical practice guideline, we focus on information needs araising during its actual application in clinical care. To this end, let us look on a variety of studies that have been investigating clinical information needs. These include studies on physicians' and nurses' information needs, but also studies on patients' information needs as outlined in the following subsections.

\subsubsection{Physicians}

Multiple studies have been conducted to investigate the information needs and information seeking behavior of physicians. In some of them these information needs are presented as general categories as Zeng and Cimino, who investigated information needs that could be met with information in the Electronic Medical Record (EMR) [108]. The derived information needs were categorized into major categories and minor categories, whereupon they decided which kind of views would best support the different data characteristics (see Table 2.5):

Table 2.5: Major and minor categories of information needs according to Zeng and Cimino [108] (the frequency gives the percentage of this category of the total number of information needs verbalized during an observational study).

\begin{tabular}{lll}
\hline Major Category & Minor Category & Frequency \\
\hline Demographics & Name, Medical Record Number, Gender... & $8,78 \%$ \\
\hline \multirow{3}{*}{ Diagnostic Procedures } & Results of specific tests & $9,46 \%$ \\
& Past results of specific tests & $4.39 \%$ \\
& Trend of tests & $3,72 \%$ \\
& Any past hospitalizations/tests (why) & $8,45 \%$ \\
& Health maintenance & $4,05 \%$ \\
& Test plan & $3,72 \%$ \\
\hline \multirow{5}{*}{ Medications and Treatments } & Past treatments & $6,76 \%$ \\
& Responses/complaints & $2,36 \%$ \\
& Current treatments & $6,43 \%$ \\
& Treatment plans & $6,08 \%$ \\
\hline & Care from other providers & $5,41 \%$ \\
& Past diagnoses & $8,11 \%$ \\
& Current diagnoses & $7,43 \%$ \\
& Evidence to rule in/out diagnoses & $3,72 \%$ \\
Past signs/symptoms of a specific diagnosis & $3,04 \%$ \\
& Allergy & $2,36 \%$ \\
Family history & $0,20 \%$ \\
& Differential diagnosis for findings & $3,72 \%$ \\
\hline
\end{tabular}

Other studies go into greater detail, providing a comprehensive list of questions as formulated by physicians. A good example of this type of survey is the research conducted by Seol et 
al. They performed a study to assess physicians' information needs expressed while reviewing clinical cases [77]. Thus, thirteen physicians reviewed three different scenarios, representing patients with cardiovascular problems. The arising questions were categorized into four topics: treatment, diagnosis, etiology, and prognosis. Questions concerning overview or review information were excluded from the analysis, which resulted in a set of 182 questions. In all three scenarios, the need for treatment information prevailed by far. Again, common question patterns were identified, whereas a small number of semantic patterns could account for a large number of questions. These patterns can be found in Table 2.6.

Table 2.6: Generic question patterns observed when thirteen physicians reviewed three clinical cases, representing patients with cardiovascular problems [77].

\begin{tabular}{|c|}
\hline Questions \\
\hline Does drug $\mathrm{X}$ (or therapeutic procedure $\mathrm{X}$ ) treat condition $\mathrm{Y}$ ? \\
\hline Is drug $\mathrm{X}$ more effective than drug X1...Xn for condition $\mathrm{Y}$ caused by organism Z? \\
\hline Does drug $\mathrm{X}$ (or therapeutic procedure $\mathrm{X}$ ) cause condition $\mathrm{Y}$ ? \\
\hline What are the side effects of drug X? \\
\hline What is the correct dose of drug X? \\
\hline Are there any interactions between drug X1 and drug X2? \\
\hline Is a low dose of drug $\mathrm{X}$ as effective as a high dose of drug $\mathrm{X}$ ? \\
\hline When should drug X be stopped/started? \\
\hline What is the treatment for condition $\mathrm{X}$ associated with condition $\mathrm{Y}$ ? \\
\hline What is the treatment for condition $\mathrm{Y}$ caused by drug $\mathrm{X}$ (or therapeutic procedure $\mathrm{X}$ )? \\
\hline When should therapeutic procedure X be stopped/started? \\
\hline How many units should be given to treat condition Y? \\
\hline Does therapeutic procedure $\mathrm{X}$ affect physiologic function $\mathrm{Y}$ ? \\
\hline Is treatment $\mathrm{X} 1$ more effective than treatment $\mathrm{X} 2 \ldots \mathrm{Xn}$ ? \\
\hline What are the causes for condition Y? \\
\hline What is the normal range of test result $\mathrm{X}$ ? \\
\hline Is condition Y1 associated with condition Y2? \\
\hline Is behavior $\mathrm{W}$ associated with condition $\mathrm{Y} 2$ ? \\
\hline Does organism $\mathrm{Z}$ cause condition $\mathrm{Y}$ ? \\
\hline Does condition $\mathrm{Y}$ affect body substance $\mathrm{X}$ ? \\
\hline What is the prognosis of condition Y? \\
\hline What is the prognosis of condition Y1 associated with Y2? \\
\hline What is the prognosis of condition $\mathrm{Y}$ complicated by therapeutic procedure $\mathrm{X}$ ? \\
\hline
\end{tabular}

Questions of a more "practical" nature were formulated by Allen et al. [1] (see Table 2.7). They conducted a study to derive clinicians' information needs while using a clinical information system (CIS). Clinicians were observed and recorded on audiotape while using a CIS; additionally, the computer screen was recorded on videotape. From the questions the clinicians asked 
during these sessions, Allen et al. derived different information needs and grouped them into categories.

- Navigation: Questions such as "What is the normal value of this laboratory test?" were associated with this category. It is called navigation, because the answers to the questions within this category were available in the given CIS. Thus, a link to navigate the user to another page is sufficient to solve these needs.

- Cross-reference: This category includes questions, such as "What laboratory tests are associated with this particular radiology reports".

- Laboratory: A question of this category could be "What is the calculated anion gap?".

- Pharmacy: "What are the different formulations of medication?" is a representative question of this category.

- Differential Diagnosis: Includes questions such as "What is the differential diagnosis of this symptom/sign/test result?".

- Definition/Information need: This category includes question such as "What does this specific term mean?".

In addition, they identified eleven specific repeated question patterns for $47 \%$ of the questions (see Table 2.7).

Table 2.7: Recurring question patterns representing $47 \%$ of all questions asked by physicians while using a clinical information system (CIS) [1].

\begin{tabular}{l}
\hline Questions \\
\hline What does the term/number/abbreviation $\mathrm{X}$ in the CIS mean? \\
\hline How do I find the normal value/trend for test $\mathrm{X}$ ? \\
\hline What is the telephone number/beeper number for attending/resident $\mathrm{X}$ ? \\
\hline What other tests are important to know when ordering/reviewing the medication/report X \\
on this patient? \\
\hline What patient educational information is available about the test/disease $\mathrm{X}$ ? \\
\hline What inpatient or outpatient medication is this patient on or has been given? \\
\hline How do you calculate finding $\mathrm{X}$ for this patient with these results? \\
\hline How do you perform procedure $\mathrm{X}$ to obtain laboratory test $\mathrm{Y} ?$ \\
\hline Is this the correct preparation of medication $\mathrm{X}$ for this patient? \\
\hline What is the differential diagnosis of sign/symptom/laboratory test/report finding $\mathrm{X}$ in this \\
patient? \\
\hline What is the definition of term $\mathrm{X}$ ?
\end{tabular}

These examples show a general difficulty inherent in exploiting these surveys: every group of researchers formulates their own specific set of questions or categories which may be but are not always interrelated. Therefore - data permitting - it is necessary to normalize these categories and questions in order to arrive at a uniform set of findings, weighted according to frequency 
and importance before proceeding with the actual generation and implementation of the desired software tool (see Section 3.2).

This is also true for various other surveys that have been found in literature. They are listed in the following paragraphs in chronological order.

Smith, 1996. Smith investigated 13 studies concerning physicians' information needs, which were conducted between 1978 and 1994. A summary of these studies can be found in Table 2.8. Despite of some deficiencies of the studies (some studies are on small populations of doctors, all but one are American, and many studies are of moderate quality [83]), Smith draws the following tentative conclusions [83]:

- Information needs arise regularly when doctors see patients.

- Questions are mostly about treatment and drugs.

- Often these questions are complex, requiring both knowledge about particular patients and knowledge about different areas of medicine.

- Doctors are not only looking for medical knowledge, but also for support, affirmation, commiseration, sympathy, judgment, and feedback.

- Most questions arising during consultation go unanswered.

- The first source doctors consult when seeking answers to questions is colleagues.

- Most of the questions can be answered from electronic sources, but it is time consuming to do so.

- Doctors seem to be overwhelmed by the enormous amount of information available.

Chambliss et al., 1996. Chambliss et al. [12] conducted a study where family physicians were asked to report unanswered questions after their half-day practice. The outcome was that the most frequently asked questions - among 103 questions collected - were about treatment $(37 \%)$ and diagnosis $(29 \%)$, but also questions about drug information occurred frequently $(15 \%)$.

Ely et al., 1999 and 2000. In 1999 Ely et al. presented a taxonomy of 69 types of questions derived from 1101 questions asked by 103 family doctors participating in their study [26]. They outlined that a set of 25 most common generic questions represented $81 \%$ of all cases. In addition, they categorised the questions, where the most common question topics were drug prescribing, obstetrics and gynaecology, and adult infectious disease. In 2000 Ely et al. conducted a subsequent study [27] with a less homogeneous group of 49 primary care doctors (29 family doctors, 14 general internists, and 6 general pediatricians) and revised the taxonomy to achive a more general reliability of their results. The ten most frequent generic questions are listed in Table A.1, mainly dealing with diagnosis, treatment, and medication. 
Table 2.8: Summary of studies focusing on physicians' information needs between 1978 and 1994 [83] (the frequency gives the percentage of this information need of the total number of information needs detected during the study).

\begin{tabular}{|c|c|c|c|c|}
\hline Study & Method & Subjects & Information need & Frequency \\
\hline Strasser 1978 [85] & Questionnaire & 258 Practicing doctors & $\begin{array}{l}\text { New developments in } \\
\text { specialty } \\
\text { Drug related } \\
\text { Cancer }\end{array}$ & NA \\
\hline $\begin{array}{l}\text { Northup et al. } \\
1983[63]\end{array}$ & $\begin{array}{l}\text { Critical incident } \\
\text { technique }\end{array}$ & $\begin{array}{l}293 \text { Medical students } \\
\text { and doctors }\end{array}$ & $\begin{array}{l}\text { Disease related } \\
\text { Drug related } \\
\text { Procedure related }\end{array}$ & $\begin{array}{l}49 \% \\
23 \% \\
19 \%\end{array}$ \\
\hline $\begin{array}{l}\text { Covell et al. } \\
1985[16]\end{array}$ & $\begin{array}{l}\text { After visit inter- } \\
\text { view }\end{array}$ & 47 Primary care doctors & $\begin{array}{l}\text { Treatment } \\
\text { Diagnosis } \\
\text { Drug related }\end{array}$ & $\begin{array}{l}31 \% \\
25 \% \\
14 \%\end{array}$ \\
\hline $\begin{array}{l}\text { Timpka et al. } \\
1989[95]\end{array}$ & $\begin{array}{l}\text { Questionnaire, } \\
\text { including critical } \\
\text { incident questions }\end{array}$ & 84 General practitioners & $\begin{array}{l}\text { General medicine } \\
\text { Dermatology }\end{array}$ & $\begin{array}{l}48 \% \\
11 \%\end{array}$ \\
\hline $\begin{array}{l}\text { Williamson et al. } \\
1989[104]\end{array}$ & Telephone survey & $\begin{array}{l}492 \text { Primary care doctors, } \\
90 \text { opinion leaders }\end{array}$ & $\begin{array}{l}\text { Drug related } \\
\text { Laboratory tests }\end{array}$ & $\begin{array}{l}38 \% \\
25 \%\end{array}$ \\
\hline $\begin{array}{l}\text { Woolf and Benson } \\
1989[105]\end{array}$ & $\begin{array}{l}\text { Administered } \\
\text { questionnaire }\end{array}$ & $\begin{array}{l}42 \text { Professors, } \\
25 \text { house staff }\end{array}$ & $\begin{array}{l}\text { Treatment } \\
\text { Differential diagnosis } \\
\text { Drug related }\end{array}$ & $\begin{array}{l}77 \% \\
75 \% \\
64 \%\end{array}$ \\
\hline $\begin{array}{l}\text { Timpka and Arborelius } \\
1990[94]\end{array}$ & $\begin{array}{l}\text { Video recordings } \\
\text { of consultations }\end{array}$ & 12 General practitioners & $\begin{array}{l}\text { Diagnosis } \\
\text { Treatment } \\
\text { Orthopaedics } \\
\text { Internal medicine }\end{array}$ & $\begin{array}{l}55 \% \\
33 \% \\
29 \% \\
26 \%\end{array}$ \\
\hline $\begin{array}{l}\text { Osheroff et al. } \\
1991[65]\end{array}$ & $\begin{array}{l}\text { Anthropological } \\
\text { observation }\end{array}$ & $\begin{array}{l}24 \text { Doctors and medical } \\
\text { students }\end{array}$ & $\begin{array}{l}\text { Specific patient } \\
\text { Treatment }\end{array}$ & $\begin{array}{l}61 \% \\
25 \%\end{array}$ \\
\hline $\begin{array}{l}\text { Ely et al. } \\
1992[25]\end{array}$ & Observation & 34 Family physicians & $\begin{array}{l}\text { Treatment } \\
\text { Drug related } \\
\text { Diagnosis }\end{array}$ & $\begin{array}{l}73 \% \\
49 \% \\
27 \%\end{array}$ \\
\hline $\begin{array}{l}\text { Bowden et al. } \\
1994[8]\end{array}$ & Questionnaire & 442 Doctors & $\begin{array}{l}\text { Treatment } \\
\text { Diagnosis } \\
\text { Drug related }\end{array}$ & $\begin{array}{l}34 \% \\
28 \% \\
18 \%\end{array}$ \\
\hline $\begin{array}{l}\text { Guise et al. } \\
1994[38]\end{array}$ & Record review & 7 Health professionals & $\begin{array}{l}\text { Treatment } \\
\text { Drug related }\end{array}$ & $\begin{array}{l}24 \% \\
18 \%\end{array}$ \\
\hline
\end{tabular}

NA: information not available from the publications.

Green et al., 2002. Green et al. investigated the information needs of physicians by interviewing 64 residents after 401 patient encounters [36]. On the average, they identified 2 questions for every 3 patients and categorized them as follows: $28 \%$ background questions (asking for general information about a disease process or patient presentation, i.e., the description of a disease, the general approach to evaluation, available therapeutic options, or possible causes of a condition), and $66 \%$ foreground questions (asking for an explicit or implied outcome resulting from a particular intervention in a patient). $38 \%$ of all questions were related to therapy, $27 \%$ to di- 
agnosis, $16 \%$ to etiology, $8 \%$ to prognosis, $7 \%$ to prevention, $6 \%$ to drug information (including cost, dosing, interactions, and adverse effects), and $4 \%$ were concerned with clinical findings. In addition, the impact of these unanswered questions on patient care was investigated with the result that physicians thought that $70 \%$ of the questions would change patient management, $34 \%$ might involve harming the patient if not answered, and $24 \%$ of the questions were urgent.

McKnight et al., 2002. McKnight et al. conducted a study at the Presbyterian Hospital campus of the New York Presbyterian Hospital [56]. In order to perceive information needs and communication difficulties among physicians and nurses, they designed a survey to which 26 physicians and 17 nurses responded. In addition, they conducted three focus group meetings with physicians and two focus group meetings with nurses which consisted of two to six participants. They recorded 64 statements about information needs and 46 statements regarding communication difficulties. Information needs were categorized into patient specific information, institution specific information, and domain specific information (see Table A.2 for physicians and Table 2.10 for nurses).

Collins et al., 2009. A recently published study by Collins et al. [14] was conducted to investigate information needs of clinicians depending on both context and clinician type. They observed 14 clinicians (six medical residents, four nurse practitioners, three registered nurses, and one physician assistant) from the New York Presbyterian Hospital between October 2006 and October 2007. The participants were instructed to verbalize their thoughts and questions while using the clinical information system (CIS). With respect to a previously elaborated Information Needs Event taxonomy [17] they identified clinical questions and characterized them according to their generic question type, clinician type, information need type, etc. A list of the total number of generic question types by clinician group and the average number by session is given in Table A.3. The frequency of the question types clearly differed in accordance with clinician type; for instance, medical residents asked for information about unfamiliar domains, while registered nurses asked about physician order rationales (results for nurses is given in Table A.7). 


\subsubsection{Nurses}

We identified several studies dealing with the information needs of nurses and nurse practitioners. Again they differ in presentation and categorization of the information needs and they use different methods to capture them, as for instance McCaughan et al., who performed an exploratory study [55] to investigate the kinds of decisions made by nurse practitioners and practice nurses as well as their use of research information in clinical decision making. McCaughan et al. used interviews, observation, and documentary analysis to collect data from 29 practice nurses and four nurse practitioners in the North of England. In doing so, they worked out a seven-fold taxonomy of the types of decisions made by the nurses on a daily basis:

Table 2.9: McCaughan et al. worked out a taxonomy of decisions made by nurses [55].

\begin{tabular}{ll}
\hline Decision type & Example \\
\hline Assessment & Decision to assess patient for risk of heart disease \\
\hline Diagnosis & $\begin{array}{l}\text { Diagnostic decisions, for instance whether a skin condition } \\
\text { is eczema }\end{array}$ \\
\hline Intervention & $\begin{array}{l}\text { Decision to intervene, for instance, prescribing antibiotics } \\
\text { for urinary tract infection }\end{array}$ \\
\hline Targeting & $\begin{array}{l}\text { Decisions about which patients should receive a particular } \\
\text { intervention, for instance, patch or bupropion to promote } \\
\text { smoking cessation }\end{array}$ \\
\hline Timing & \begin{tabular}{l} 
Decisions concerning the scheduling of travel injections \\
\hline Prevention
\end{tabular} \\
$\begin{array}{l}\text { Decision to give advice on methods of contraception to pre- } \\
\text { vent pregnancy and spread of sexually transmitted disease }\end{array}$ \\
\hline Communication & $\begin{array}{l}\text { Decision to communicate risks and benefits associated with } \\
\text { different types of malaria prophylaxis }\end{array}$ \\
\hline Referral & $\begin{array}{l}\text { Decision to refer patients to the general practitioner, dieti- } \\
\text { tian, specialist nurse }\end{array}$ \\
\hline Service delivery & $\begin{array}{l}\text { Decision to set up a new clinic for patients with chronic } \\
\text { obstructive pulmonary disease }\end{array}$ \\
\hline seeking & $\begin{array}{l}\text { Decision to look for information about an aspect of practice, } \\
\text { for example, concerning MMR vaccination }\end{array}$ \\
\hline
\end{tabular}

In addition, McCaughan et al. investigated the sources of information used by the nurses in clinical decision making. They revealed that most nurses relied on personal experience or they asked general practitioner or other nurses for advise because they are immediately available and viewed as reliable. Moreover, nurses used commercially sponsored information sources, such as commercial representatives and sponsored 'nurse advisors'; both of which should be rather used with caution. As far as protocols and clinical practice guidelines are concerned, nurses gave the following reasons for rarely using them [55]:

- Guidelines and protocols were not viewed as particularly useful for practice nor accessible.

- Nurses believed guidelines were describing routine practice that was already familiar, rather than providing direction when an unforeseen need for information arose.

- It was usually quicker and easier to ask a colleague. 
- Due to a lack of IT skills, computerized protocols were inaccessible for many nurses.

- Often protocols were not up-to-date evidence.

- Nurses reported that protocols did not supply them with clear messages for practice.

- Opportunities to develop written protocols were limited.

However, there was recognition that protocols and guidelines might be useful to reach consistency of treatment across the different members of the primary care team as long as everyone would adhere to them.

Other studies focus on information needs, as for instance McKnight et al., who conducted a study [56] (see Section 2.3.1) to investigate information needs and communication difficulties between 26 physicians and 17 nurses at the Presbyterian Hospital campus of the New York Presbyterian Hospital. Moreover, they conducted two focus group meetings with nurses which consisted of two to six participants. They roughly categorized 64 statements about information needs and 46 statements regarding communication difficulties as given in Table 2.10. Obviously these categories differ very much from the categories of decisions made by the nurses as reported by McCaughan et al. [55].

Table 2.10: Information needs and communication difficulties perceived by nurses (McKnight et al. [56]).

\begin{tabular}{|c|c|}
\hline Field & Information Need \\
\hline \multirow{2}{*}{ Patient Specific } & Patient diagnosis \\
\hline & Laboratory and other test results \\
\hline \multirow[t]{2}{*}{ Institution Specific } & $\begin{array}{l}\text { Policies and protocols (IV access device care policy, blood bank } \\
\text { protocol) }\end{array}$ \\
\hline & Census reports \\
\hline \multirow{3}{*}{ Domain Specific } & $\begin{array}{l}\text { Drug information (dosage and side effects of specific drugs, pa- } \\
\text { tient/caregiver teaching information) }\end{array}$ \\
\hline & Diagnostic definitions \\
\hline & Educational material (e.g. colostomy care educational material) \\
\hline Communication Difficulties & Identifying and contacting other health care providers \\
\hline
\end{tabular}

Based on the findings of this study, they came to the conclusion that physicians and nurses perceive significant gaps between information needs and timely access. Furthermore, they have different needs, methods and goals, but they share common problems in obtaining information and communication effectively.

Other studies worked out more concrete lists of generic questions. An example of this type is the study conducted by $\mathrm{Xu}$ et al. [107]. They investigated nurses' information needs and searching behavior in acute care settings. They conducted a study in three inpatient acute care units, which took about four months. Nurses from these three sites were trained in using a web-based tool called CPG Viewer [106], which allows for browsing a CPG collection. They monitored the interactions with the tool in daily routine; after each session the nurses were asked to fill out a questionnaire regarding what information $\mathrm{s} /$ he was looking for and if $\mathrm{s} / \mathrm{he}$ was successful in finding the correct information. The recorded data about the interaction with the CPG Viewer and the questionnaire answers were used to analyze the information seeking 
behavior of the nurses. Information needs that were frequently formalized in the questionnaires can be found in Table 2.11.

Table 2.11: Frequency of generic questions identified by Xu et al. [107].

\begin{tabular}{ll}
\hline Question & Frequency \\
\hline What is the protocol or procedure for condition X? & $48,15 \%$ \\
\hline What do I assess for X? & $11,11 \%$ \\
\hline What are the symptoms of X? & $7,41 \%$ \\
\hline What is the definition of X? & $6,17 \%$ \\
\hline What are the lab values for X? & $6,17 \%$ \\
\hline What do I need to document for X? & $4,94 \%$ \\
\hline What are the risk factors for X? & $4,94 \%$ \\
\hline What is the treatment of X? & $2,47 \%$ \\
\hline What is the physiology of X? & $1,23 \%$ \\
\hline
\end{tabular}

Based on the results of the study, $\mathrm{Xu}$ et al. reason that nurses' information needs differ from physicians' information needs as reported by Ely et al. [27] (see Section 2.3.1 and Table A.1). About half of the information needs formulated by the nurses were about protocols and procedures, while the study of Ely et al. showed that physicians are frequently seeking information about choices of drugs and cause of symptoms. However, both groups of healthcare providers needed information about the treatment for a given condition.

Other surveys that have been found in literature are listed in the following paragraphs in chronological order.

Blythe, 1993. Blythe [7] conducted a study to find out why nurses neglected library resources even though studies have shown that using the library had a significant positive effect on patient treatment. Reasons for this behavior were the overwhelming volume of information, ignorance of searching techniques, a lack of time, and problems with the library hours. 32 nurses of a general medical and hematology and oncology unit in a teaching hospital filled out questionnaires; in addition, 16 staff nurses and other unit personnel were interviewed. The results of this study showed that the nurses used information sources like fellow nurses, physicians, and other members of the health care team, but also individual patient records, the Kardex ${ }^{4}$, institution specific publications, departmental manuals, commercially published reference sources (e.g. the Compendium of Pharmaceuticals and Specialties - CPS), laboratory reports, and other resources. Most nurses used the patient's record and the Kardex on a daily basis and laboratory results were used frequently.

Moreover, the study showed that non-routine questions were often more complex to solve and required the collection of information from multiple sources, for instance, a drug related question might require input from a patient's record, laboratory reports, the CPS, and other sources. Nurses stated in both interviews and questionnaires that their greatest information need is for accurate, concise reference sources in either printed books or computerized databases.

\footnotetext{
${ }^{4}$ Kardex: "trademark for a card-filing system that allows quick reference to the particular needs of each patient for certain aspects of nursing care. Included on the card may be a schedule of medications, level of activity allowed, ability to perform basic self-care, diet, any special problems, a schedule of treatments and procedures, and a care plan. The Kardex is updated as necessary and is usually kept at the nurses' station" [57].
} 
Moreover, they stressed the fact, that they need direct access on the unit itself because they cannot leave their patients.

Cogdill, 2003. Cogdill published the results of his study [13] of the information related behavior of nurse practitioners in North Carolina. In the first phase of data collection, Cogdill sent out a questionnaire to 300 nurse practitioners (134 were returned), who were asked to report their information needs during patient encounters as well as their information seeking behavior. Results of the questionnaires can be found in Table A.4 and Table A.5; the former lists the frequency of information needs and the latter lists the frequency of information resources use reported by the nurses in the questionnaire (e.g., most frequently used information resources are primary supervising physicians and drug reference manuals). Drug therapy and diagnosis are the categories of the most frequently encountered information needs, but also information needs of the categories etiology, disposition, and epidemiology were stated in the questionnaire.

In the second phase of data collection a series of interviews were conducted with 20 nurse practitioners after their encounters with patients. The total number of information needs reported in the interviews was 75 and the average information need per patient was 0,57 needs. Table A.6 shows the frequency of information needs reported with respect to the category of information. Again, drug therapy and diagnosis are the categories of the most frequent information needs, but - in contrast to the results of the questionnaire - information needs of the categories general management and prognosis were reported, and in turn the categories etiology, disposition, and epidemiology are negligible.

Moreover, the analysis of both results showed that the number of information needs reported in the interviews after the patient encounters was significantly higher than the number of information needs reported in the questionnaires, which indicates that the nurses might not be aware of the extend to which they seek for information.

Collins et al., 2009. Results of the study published by Collins et al. [14] (see Section 2.3.1) regarding four nurse practitioners and three registered nurses from the New York Presbyterian Hospital are given in Table A.7. They observed the participants between October 2006 and October 2007 while using the clinical information system and identified clinical questions. The most frequently asked questions are "How is this done at this institution?", "What is the patient education related to X?", and "Can drug X cause adverse finding Y?". However, due to the very small number of participants the results are not very representative.

In the previous section we have listed diverse results of studies on the information needs of nurses and nurse practitioners; in Section 3.2.2 we give a detailed outline of normalized findings of these studies. 


\subsubsection{Patients}

To be able to make informed decisions, patients need sufficient, appropriate, and detailed information about conditions, different treatments, likely outcomes, risks, and influences on their quality of life [15]. There is evidence in literature that patients often experience difficulties to understand the information that is given to them and that they want more information than they receive $[4,18,50]$. Thus, before introducing information to patients, it is crucial to understand patients' information needs and in which way the information should be given to them. Coulter et al. provide a list of reasons why patients need to be informed adequately. Patients need information to [15]

- Understand what is wrong

- Gain a realistic idea of prognosis

- Make the most of consultations

- Understand the processes and likely outcomes of possible tests and treatments

- Assist in self care

- Learn about available services and sources of help

- Provide reassurance and help to cope with their illness

- Help others understand

- Legitimize seeking help and their concerns

- Learn how to prevent further illness

- Identify further information and self help groups

- Identify the "best" healthcare providers

They surveyed patients which were recruited via newspaper advertisements and self help groups [15]. All participants had recent experience with one of ten specific health problems (back pain, cataract, depression, glue ear, high cholesterol, hip replacement, infertility, menorrhagia, prostate enlargement, and stroke rehabilitation) and they discussed their information needs in ten focus groups (one for each health problem). Based on the analysis of the discussions Coulter et al. stated: "Patient focus groups reported considerable dissatisfaction with their experiences of communication with health professionals. Most had wanted much more information about their condition and treatment than they had been given. Many did not feel they had been offered any choices about their treatment, and some had not realized that there were other options until they received the information materials we sent them for review." [15].

To understand patients' information needs adequately, Coulter et al. investigated these needs in particular. They provide a list of the most commonly asked questions by patients [15]:

Other studies focused on the publication of patient education materials, i.e., what information should be given and how to formulate the information. The NetReach project [87] was aimed at determining information needs of physicians in diverse ambulatory care settings on the campus of the Northwestern Memorial Hospital in Chicago as well as implementing information tools at the test sites. Care settings included primary care internal medicine clinics, specialty care clinics, faculty practice clinics, independent private practices, and an urban community clinic. One of the results of the physicians' information needs assessment was that physicians 
Table 2.12: Most commonly asked questions by patients (Coulter et al. [15]).

\begin{tabular}{l}
\hline Questions \\
\hline What is causing the problem? \\
\hline Am I alone? How does my experience compare with that of other patients? \\
\hline Is there anything I can do myself to ameliorate the problem? \\
\hline What is the purpose of the tests and investigations? \\
\hline What are the different treatment options? \\
\hline What are the benefits of the treatment(s)? \\
\hline What are the risks of the treatment(s)? \\
\hline Is it essential to have treatment for this problem? \\
\hline Will the treatment(s) relieve the symptoms? \\
\hline How long will it take to recover? \\
\hline What are the possible side effects? \\
\hline What effect will the treatment(s) have on my feelings and emotions? \\
\hline What effect will the treatment(s) have on my sex life? \\
\hline How will it affect my risk of disease in the future? \\
\hline How can I prepare myself for the treatment? \\
\hline What procedures will be followed if I go to hospital? \\
\hline When can I go home? \\
\hline What do my carers need to know? \\
\hline What can I do to speed recovery? \\
\hline What are the options for rehabilitation? \\
\hline Where can I prevent recurrence or future illness? \\
\hline
\end{tabular}

across the diverse sites spend approximately $60 \%$ of their time on talking and $37 \%$ of this talking-time on patient education and instructions. Despite this significant effort, the literature showed that conventional patient education has not been effective $[11,52,74]$, which led them to adress this problem in particular. Thus, Tang et al. conducted a patient focus group study and published a set of desired characteristics of patient education materials (ranked in order of importance) [88]:

1. is quick to use

2. presents material clearly

3. contains information on diseases

4. provides follow-up instructions 
5. allows user to add new topics

6. allows user to customize content

7. written at the 6 th grade reading level for ease of understanding

8. easy to use

9. contains information on preventative medicine topics

10. contains information on medications

11. contains references for further information

12. available in Spanish (probably due to the proportion of Hispanics of the population)

13. uses graphics

Furthermore, they stated additional desired characteristics; the patient education materials should:

- be custom-tailored to the patient's situation

- be well organized, concise, and practical

- be printed so that the patients can take the materials with them

- include personal health data as a record of their health

- be reviewed with the individual patient by the physician

- help the patients to formulate relevant questions

- include follow up references for more information

Besides, there are studies which reveal that patients need information not only about their disease, treatment, and prognosis, but also about very practical and personal issues, such as impact on quality of life and handling the emotional impact. A good example for this kind of information needs results form the study conducted by Wallberg et al. [102], who investigated the information needs of Swedish breast cancer patients as well as their desire to participate in the decision process. They interviewed 201 patients on nine categories of information and five patient roles, using paired comparisons. The nine categories of information needs have been identified in a literature review by Degner et al. [22]. Subsequently, the patients ranked the categories of information needs according to priority which resulted in a profile of important information needs for women with breast cancer (ranked according to priority) [102]:

1. Disease cure: Information about the likelihood of cure from my disease.

2. Disease stage: Information about the diagnostic stage and the extent of involvement of my disease.

3. Treatment risks: Information about different types of treatments (surgical, chemo-therapy, radiotherapy) and the possible benefits and risks associated with each treatment.

4. Side effects: Information about possible unpleasant side effects of treatment (nausea, pain, change in physical appearance). 
5. Family risk: Information about how at risk my children and/or other family members are in developing the disease.

6. Social life: Information about how the treatment may affect my ability to carry on my usual social activities (sports, hobbies, etc).

7. Self care: Information about caring for myself at home (nutrition, support groups, home care, social workers, mental health workers, etc).

8. Family impact: Information about how to handle the physical and emotional impact of the disease on my family and others.

9. Attractiveness: Information about how the treatment may affect my usual feelings of physical and sexual attractiveness (breast disfigurement, breast prothesis, reconstructive surgery).

Moreover, we found a variety of interesting studies focusing either on general information needs of patients, on information needs of patients with a specific type of illness, on desired characteristics of published information materials, or on what information do patients need about medicines as outlined in the following paragraphs in chronological order.

Richardson and Moran, 1995. Richardson and Moran addressed the need to develop improved standards for patient information [71]. The Taunton and Somerset NHS Trust's Quality Assurance Steering Group conducted a review of all written patient information currently in use. Based on the analysis of these, they developed some standards for the publication of patient information with respect to the recommendations stated by the Audit Commission [4]. They distinguished between information about conditions or types of illness and information about certain specialties (see Appendix A.3). The standard condition-specific patient information is thought to include for instance, a description of the condition, different treatment options, risks and benefits of treatment, and dos and don'ts for the patient.

Furthermore, they recommend to include information such as a description of the specialty and the available treatments, directions how to reach the specialty, available facilities, and instructions about pre-attendance preparations into the specialty-specific patient information.

The developed standards also contain instructions on how to formulate the information. It should [71]:

- be clear, informal and understandable to the lay person (simple words, short sentences),

- be free of medical jargon,

- show date of production and date for review, and

- have been checked for ease of reading score or Fog Index [41].

Meredith et al., 1996. Meredith et al. interviewed 250 cancer patients in west Scotland (at the Beatson Oncology Centre, the Western Infirmary, or Glasgow Royal Infirmary) to find out about their information needs [59]. The patients - which all had been told their diagnosis just recently - were asked to fill out a questionnaire regarding their general preference for information, their chances of cure, details of all possible treatments, details of all possible side effects, etc. Patients were asked to distinguish between information they really need to know, information they would like to know, and information they would prefer not to know. Table A.8 
shows that the majority of patients absolutely want to be informed about all possible side effects of a treatment, whether their illness is cancer, what the chances of cure are, and all possible treatment options.

Dickinson et al., 2003. Dickinson et al. addressed the question what information do patients need about medicines [23]. Thus, they interviewed people from the perspectives of patients, clinicians, and the pharmaceutical industry; this resulted in formulating four essential aspects of drug treatments [23]:

1. Side effects

2. What it does and what it is for

3. Do's and don'ts

4. How to take it

Furthermore, they state that the ideal source of drug information would be [23]

- Accurate, up to date, reliable, and practically useful

- Accessible in language, format, and tone

- Capable of customization or personalization

- Available at different levels of detail at different times

- Informative about conditions as well as treatments

- Striking a balance between a treatment's beneficial and adverse effects

- Available at the time of a consultation and consistent with best advice

- Linked to other reliable and consistent sources of advice and information.

Kerr et al., 2005. Kerr et al. investigated what patients require from interactive health communication applications for chronic disease [49]. Thus, they conducted a study with diseasespecific focus groups of people who either had a long-term illness, or were caring for someone with a long-term illness (e.g. coronary heart disease, diabetes, hepatitis C, Alzheimer's disease, or asthma). The participants investigated three different interactive health communication applications and subsequently, held a focus group discussion about the strengths and weaknesses of each application and whether, when, and why they would use it. From this discussion Kerr et al. derived a list of quality criteria for interactive health communication applications. A good application should provide information about the aspects such as medication, practicalities of day-to-day living, available treatments, etc. (see Appendix A.3).

Jones et al., 2006. Jones et al. investigated the effects of different forms of information given to cancer patients [45]. They asked cancer patients to choose up to ten sections from 78 sections from specific cancer information booklets. Table A.9 and Table A.10 show the results for breast cancer and prostate cancer patients. The sections mostly chosen by breast cancer patients are healthy eating, possible causes of the illness, and side effects of radiotherapy, while prostate cancer patients wanted to know about side effects of radiotherapy, radiotherapy in general, and the reason of described therapies. 
Selman et al., 2009. Selman et al. [76] interviewed 90 patients and 38 family caregivers of four palliative care services in South Africa and one in Uganda to explore their information needs. The patients suffered from cancer, HIV infection, and motor neurone disease. Besides the finding that these patients are often uninformed about their disease and its management, which lowers their ability to cope with the disease and plan for the future, Selman et al. identified three key areas of information needs [76]:

- Causes and symptoms

- Progression, treatment, and management of disease

- Financial and social support 
Chapter 3

Categorization of the Findings 


\subsection{Formalization of a CPG}

The main task of a guideline modeler is to create a formal guideline representation model of the original narrative guideline. To support a modelers task of creating a formal model, he/she needs to know (1) of which components the target representation format is composed and (2) in which way scheduling constraints are to be modeled:

(1) Components: The investigated guideline representation models are composed of plans (sometimes also called guidelines, templates, or maps). A plan can be decomposed into

- Actions: The modeling primitives which represent the actual tasks described by clinical guidelines (e.g. medication prescriptions and clinical investigations).

- Decisions: the investigated guideline representation languages use different decision models to formalize the decisions intrinsic to the recommendations of a clinical guideline (for a detailed comparison see [67]).

- States: a plan component that defines specific scenarios, i.e. patient states and execution states. Patient states are characterized by a patient's condition (e.g. hypertension) and are often used to facilitate the automatic entry into appropriate guidelines. Execution states are characterized by the current condition of a specific plan (e.g. in Asbru started, suspended, reactivated, aborted, or completed).

- Subplans: Usually guideline plans can be decomposed into subplans (also called subguideline, task, protocol, or composite action), which in turn may consist of various subplans. The nesting of plans and subplans leads to a hierarchical structure of guideline plans.

- Branch and synchronization steps: are used to model parallel treatment options in the guideline. In some cases (PROforma and Asbru), parallel and sequential plan executions are modeled without branch and synchronization steps (e.g. by plan characteristics).

(2) Scheduling constraints: guidelines may involve sequential, parallel, iterative, and concurrent care processes. Most guideline models use flowchart-like representations to define the execution order of plans, i.e., sequential, parallel, and cyclical execution. Other models use more precise scheduling constraints (PROforma) or complex time annotations (Asbru) to manage the task execution. Decision models are also used to govern aspects of control flow.

\subsubsection{Model-Centric Formalization}

We could identify two descriptions in literature of how to model a guideline representation model in a model-centric way, namely the SAGE project and the GLIF model. These descriptions allow the derivation of the following tasks (see Section 2.2.2):

1. Identification of relevant guideline knowledge

2. Disambiguation of guideline knowledge

3. Specification of a logical and process structure of the guideline

4. Identification of clinical concepts, fundamental parameters, and their interrelationships

5. Generation of detailed data models of the previously identified concepts and parameters 
6. Generation of a mapping of the terms in the knowledge base to the terms of the specific institution

7. Modeling of the guideline knowledge by means of recommendations sets and activity graphs

8. Encoding the model in the XML format

\subsubsection{Document-Centric Formalization}

We have investigated various approaches to formalize a clinical practice guideline in a documentcentric way. These approaches employ different (and not always coherent) methods to do so. Some formalization steps are part of each of the different modeling-approaches, for instance, the marking up of relevant parts of the guideline text; others are only mentioned once, for instance, replacing passive voice by active voice. This does not necessarily mean that these steps are not part of other approaches, too, but they are not explicitly mentioned in the description of these approaches. In order to arrive at a comprehensive representation of the formalization process, we have categorized and listed all the steps mentioned. Based on the various findings about document-centric formalization methods, as outlined in Section 2.2.2, we have generated a table giving a complete overview of every conceivable detail that might be considered important in transforming a textual CPG into a guideline representation model using a document-centric approach (see Table 3.1). This list serves as a check-list to improve existing methods, and even more importantly - as a comprehensive reference to decide about required functionality of a software tool to support the document-centric formalization process.

Table 3.1: Categorization of document-centric formalization process (continued on next page).

\begin{tabular}{|c|c|c|}
\hline Category & Subcategory & Element \\
\hline Preprocessing & $\begin{array}{l}\text { Converting document into input } \\
\text { format (Stepper) }\end{array}$ & \\
\hline \multirow{3}{*}{ Selecting relevant parts } & $\begin{array}{l}\text { Excluding irrelevant parts } \\
\text { (LASSIE, Stepper) }\end{array}$ & \\
\hline & $\begin{array}{l}\text { Marking up of relevant } \\
\text { parts/sentences (DELT/A / MHB, } \\
\text { Gem-Cutter, LASSIE, Stepper) }\end{array}$ & \\
\hline & $\begin{array}{l}\text { Arranging relevant sentences } \\
\text { in a hierarchical structure } \\
\text { (DELT/A / MHB, LASSIE) }\end{array}$ & \\
\hline \multicolumn{3}{|c|}{$\begin{array}{l}\text { Marking up of knowledge elements } \\
\text { (such as recommendations, } \\
\text { definitions, actions) } \\
\text { (DELT/A / MHB, Stepper) }\end{array}$} \\
\hline \multirow{3}{*}{ Making elements independent } & $\begin{array}{l}\text { Removing unnecessary words } \\
\text { (Gem-Cutter) }\end{array}$ & \\
\hline & $\begin{array}{l}\text { Adding implicit knowledge } \\
\text { (Stepper) }\end{array}$ & \\
\hline & $\begin{array}{l}\text { Reducing decision variables to } \\
\text { prototypic nouns (Gem-Cutter) }\end{array}$ & \\
\hline
\end{tabular}


Table 3.1: Categorization of document-centric formalization process (continued on next page).

\begin{tabular}{|c|c|c|}
\hline Category & Subcategory & Element \\
\hline \multirow[t]{2}{*}{$\begin{array}{l}\text { Making elements independent } \\
\text { (continued) }\end{array}$} & $\begin{array}{l}\text { Changing passive to active voice } \\
\text { (Gem-Cutter) }\end{array}$ & \\
\hline & $\begin{array}{l}\text { Stating actions and directives } \\
\text { (Gem-Cutter) }\end{array}$ & \\
\hline \multirow{16}{*}{ Modeling clinical actions } & $\begin{array}{l}\text { Decomposing actions into atomic } \\
\text { actions (DELT/A / MHB, LASSIE) }\end{array}$ & \\
\hline & \multirow{5}{*}{ Defining important information } & ID (DELT/A / MHB, LASSIE) \\
\hline & & Annotations (LASSIE) \\
\hline & & Treatment instruments (LASSIE) \\
\hline & & $\begin{array}{l}\text { Dosage of drug administration } \\
\text { (LASSIE) }\end{array}$ \\
\hline & & Conditions (LASSIE) \\
\hline & $\begin{array}{l}\text { Classifying actions and negative } \\
\text { actions (LASSIE) }\end{array}$ & \\
\hline & $\begin{array}{l}\text { Grouping actions } \\
\text { (DELT/A / MHB, LASSIE) }\end{array}$ & \\
\hline & $\begin{array}{l}\text { Specifying hierarchical nesting } \\
\text { (DELT/A / MHB, LASSIE) }\end{array}$ & \\
\hline & \multirow{4}{*}{ Defining relations between actions } & Succeeding actions (LASSIE) \\
\hline & & Preceding actions (LASSIE) \\
\hline & & Overlapping actions (LASSIE) \\
\hline & & $\begin{array}{l}\text { Decision branches } \\
\text { (DELT/A / MHB) }\end{array}$ \\
\hline & \multirow{3}{*}{ Specifying execution order } & Parallel actions (DELT/A / MHB) \\
\hline & & $\begin{array}{l}\text { Sequential actions } \\
(\mathrm{DELT} / \mathrm{A} / \mathrm{MHB})\end{array}$ \\
\hline & & $\begin{array}{l}\text { Repeating actions } \\
\text { (DELT/A / MHB, LASSIE) }\end{array}$ \\
\hline \multirow{4}{*}{ Specifying data variables } & $\begin{array}{l}\text { Abstracting data variables } \\
\text { (DELT/A / MHB) }\end{array}$ & \\
\hline & \multirow{3}{*}{ Defining parameters } & Data definition (DELT/A / MHB) \\
\hline & & Data usage (DELT/A / MHB) \\
\hline & & Data input (DELT/A / MHB) \\
\hline \multirow{4}{*}{ Modeling temporal aspects } & \multirow{4}{*}{ Defining parameters } & Start (DELT/A / MHB) \\
\hline & & End (DELT/A / MHB) \\
\hline & & $\begin{array}{l}\text { Duration of actions } \\
\text { (DELT/A / MHB, LASSIE) }\end{array}$ \\
\hline & & $\begin{array}{l}\text { Iteration of actions } \\
\text { (DELT/A / MHB, LASSIE) }\end{array}$ \\
\hline
\end{tabular}


Table 3.1: Categorization of document-centric formalization process (end).

\begin{tabular}{|c|c|c|}
\hline Category & Subcategory & Element \\
\hline \multirow{3}{*}{ Defining required resources } & & $\begin{array}{l}\text { Personnel resources } \\
\text { (DELT/A / MHB) }\end{array}$ \\
\hline & & Devices (DELT/A / MHB) \\
\hline & & Financial costs (DELT/A / MHB) \\
\hline \multirow{13}{*}{ Modeling additional information } & \multirow{8}{*}{ Modeling background information } & $\begin{array}{l}\text { Evidence for a statement } \\
\text { (DELT/A / MHB) }\end{array}$ \\
\hline & & Purpose (DELT/A / MHB) \\
\hline & & Effects (DELT/A / MHB) \\
\hline & & $\begin{array}{l}\text { Educational information } \\
\text { (DELT/A / MHB) }\end{array}$ \\
\hline & & $\begin{array}{l}\text { Explanations (DELT/A / MHB, } \\
\text { Gem-Cutter) }\end{array}$ \\
\hline & & $\begin{array}{l}\text { Measures to define the } \\
\text { success of following the guideline } \\
\text { (DELT/A / MHB) }\end{array}$ \\
\hline & & Quality (Gem-Cutter) \\
\hline & & $\begin{array}{l}\text { Recommendation strength } \\
\text { (Gem-Cutter) }\end{array}$ \\
\hline & \multirow{3}{*}{ Modeling patient related aspects } & Risks (DELT/A / MHB) \\
\hline & & Discomfort (DELT/A / MHB) \\
\hline & & $\begin{array}{l}\text { Health prospective } \\
\text { (DELT/A / MHB) }\end{array}$ \\
\hline & \multirow{2}{*}{ Stating source statement status } & Narrative text (DELT/A / MHB) \\
\hline & & $\begin{array}{l}\text { Distinct recommendation } \\
(\mathrm{DELT} / \mathrm{A} / \mathrm{MHB})\end{array}$ \\
\hline \multirow[t]{2}{*}{ Effecting disambiguation } & $\begin{array}{l}\text { Disambiguating formulations } \\
\text { (Gem-Cutter) }\end{array}$ & \\
\hline & $\begin{array}{l}\text { Handling vague formulations } \\
\text { (Gem-Cutter, Stepper) }\end{array}$ & \\
\hline \multirow[t]{2}{*}{ Building vocabularies } & $\begin{array}{l}\text { Standardizing terms (Stepper, } \\
\text { LASSIE) }\end{array}$ & \\
\hline & $\begin{array}{l}\text { Replacing generic linguistic } \\
\text { expressions by standardized } \\
\text { terms (Stepper) }\end{array}$ & \\
\hline \multicolumn{3}{|l|}{$\begin{array}{l}\text { Verifying rule set completeness } \\
\text { (Gem-Cutter) }\end{array}$} \\
\hline \multirow{3}{*}{ Building executable statements } & $\begin{array}{l}\text { Adapting statements to structure of } \\
\text { target representation (Stepper) }\end{array}$ & \\
\hline & $\begin{array}{l}\text { Expressing statements in statement } \\
\text { logic (Gem-Cutter) }\end{array}$ & \\
\hline & $\begin{array}{l}\text { Exporting statements into target } \\
\text { representation (LASSIE, Stepper) }\end{array}$ & \\
\hline
\end{tabular}


For a clearly arranged representation of the formalization process, we give a tentative algorithmic visualization of the process in Figure 3.1. This representation is linked to the Table 3.1 above, which lists details of the single formalization steps.

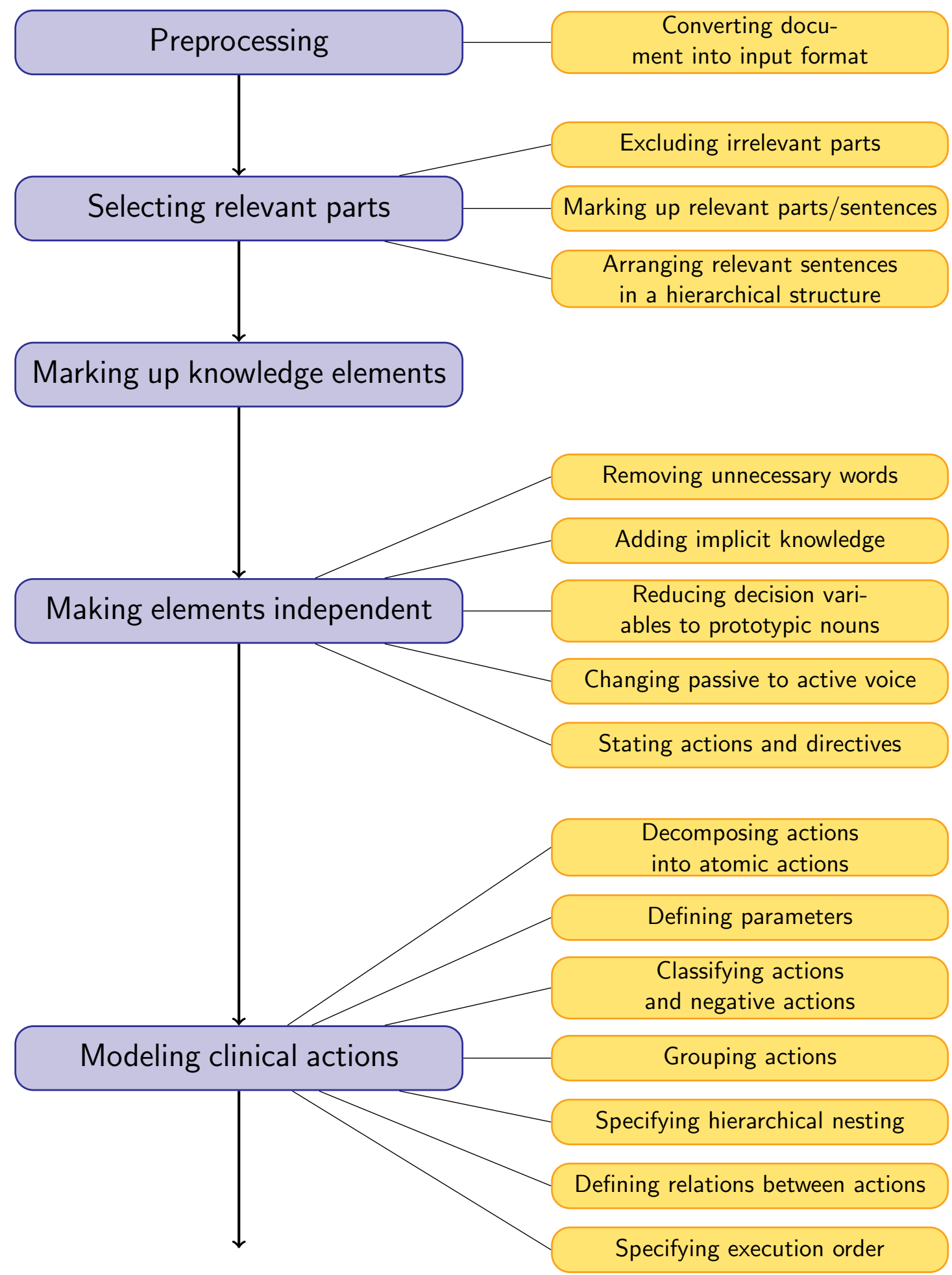

Figure 3.1: Tasks of document centric guideline formalization (continued on next page). 


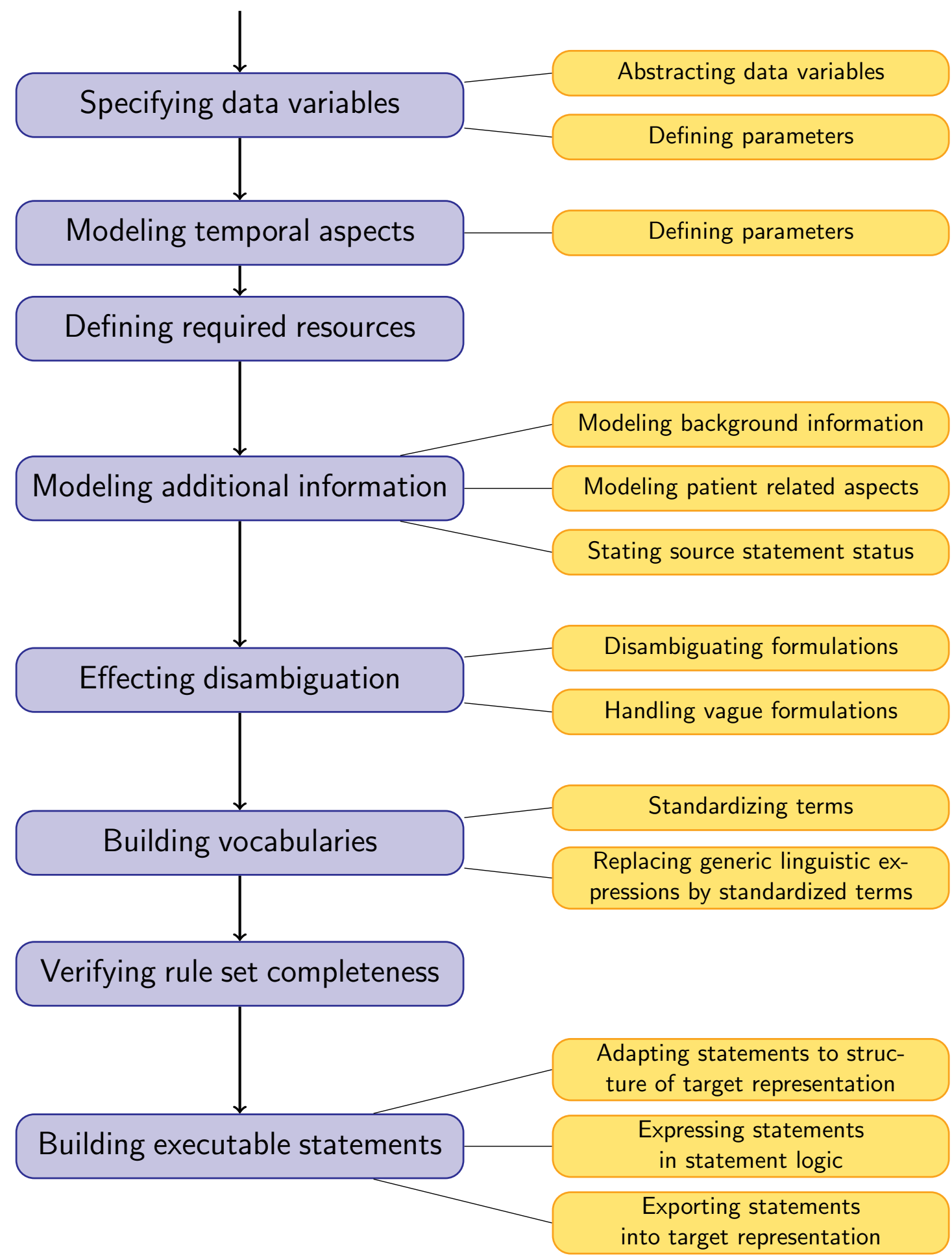

Figure 3.1: Tasks of document centric guideline formalization (end). 


\subsection{Clinical Information Needs}

The preceding section of his paper (Section 3.1) deals with technical aspects of the guideline modeling process; the following pages deal with user expectations and requirements that have to be considered in creating a clinicially useful software environment.

\subsubsection{Physicians' Information Needs}

It is to be expected that physicians will be the ones who will most frequently work with such a tool. As regards the information sought by physicians, a number of studies has been conducted, mainly in the form of retrospective questionnaires, interviews, self-reports, and observation. Table 3.2 represents an effort to give a unified picture of the information needs, Table 3.3 of the questions formulated by the physicians.

Table 3.2: Unification of physicians' information needs (continued on next page).

\begin{tabular}{|c|c|c|}
\hline Category & 1st Subcategory & 2nd Subcategory \\
\hline \multirow{21}{*}{ Information on patient data } & \multirow{3}{*}{ Demographics } & Name \\
\hline & & Gender \\
\hline & & Medical Record Number \\
\hline & \multicolumn{2}{|l|}{ Known allergies/intolerances } \\
\hline & \multirow{8}{*}{ Current state } & Symptoms \\
\hline & & Diagnosis \\
\hline & & Treatment \\
\hline & & Medication \\
\hline & & Responses/Complaints \\
\hline & & Test results \\
\hline & & Trend of test results \\
\hline & & Test plan \\
\hline & \multirow{7}{*}{ Medical history } & Past diagnoses \\
\hline & & $\begin{array}{l}\text { Past symptoms of a specific } \\
\text { diagnosis }\end{array}$ \\
\hline & & Past hospitalizations \\
\hline & & Past treatments \\
\hline & & Past medication \\
\hline & & Past test results \\
\hline & & Specialty consultations \\
\hline & \multicolumn{2}{|l|}{ Family history } \\
\hline & \multicolumn{2}{|l|}{ List of current providers } \\
\hline
\end{tabular}


Table 3.2: Unification of physicians' information needs (end).

\begin{tabular}{|c|c|c|}
\hline Category & 1st Subcategory & 2nd Subcategory \\
\hline \multirow{7}{*}{ Information relevant for diagnosis } & \multirow{6}{*}{ Findings/Conditions } & Possible causes \\
\hline & & Calculation \\
\hline & & Relations between findings \\
\hline & & Effects of findings \\
\hline & & Indicated tests \\
\hline & & Normal values of test results \\
\hline & Differential diagnoses & \\
\hline \multirow{4}{*}{$\begin{array}{l}\text { Information about } \\
\text { disease/condition }\end{array}$} & Definition & \\
\hline & Description & \\
\hline & Etiology & \\
\hline & Prognosis & \\
\hline \multirow{16}{*}{$\begin{array}{l}\text { Information on management } \\
\text { of disease/condition }\end{array}$} & \multirow{3}{*}{ Therapeutic options } & $\begin{array}{l}\text { Recommended treatment for } \\
\text { specific condition }\end{array}$ \\
\hline & & All treatment options \\
\hline & & Available treatment options \\
\hline & \multirow{3}{*}{ Treatment } & Institution specific procedure \\
\hline & & Scheduling \\
\hline & & Indicated tests \\
\hline & \multirow{3}{*}{ Risks and benefits } & Adverse effects \\
\hline & & Efficiency for specific condition \\
\hline & & Costs \\
\hline & \multirow{6}{*}{ Medication } & $\begin{array}{l}\text { Recommended medication for } \\
\text { specific condition }\end{array}$ \\
\hline & & Risks and benefits \\
\hline & & Interactions \\
\hline & & How to administer drug \\
\hline & & Dosage \\
\hline & & Scheduling \\
\hline & Instructions for patient & \\
\hline \multirow{2}{*}{$\begin{array}{l}\text { Information about other } \\
\text { healthcare providers }\end{array}$} & On-call current providers & Contact data \\
\hline & Specialty & Contact data \\
\hline References & Disease & \\
\hline
\end{tabular}


We have merged - from different sources $[1,14,26,27,77]$ - the most common generic questions asked by physicians; they are listed in Table 3.3 .

Table 3.3: The most common generic question patterns of physicians' information needs $[1,14$, $26,27,77]$ (continued on next page).

\begin{tabular}{|c|c|}
\hline Questions & \\
\hline \multirow{11}{*}{ Treatment } & What is the treatment for condition $\mathrm{X}$ associated with condition $\mathrm{Y}$ ? \\
\hline & Does therapeutic procedure $\mathrm{X}$ treat condition $\mathrm{Y}$ ? \\
\hline & $\begin{array}{l}\text { What is the treatment for condition } \mathrm{Y} \text { caused by drug } \mathrm{X} \text { (or therapeutic pro- } \\
\text { cedure } \mathrm{X} \text { )? }\end{array}$ \\
\hline & When should therapeutic procedure X be stopped/started? \\
\hline & How many units should be given to treat condition $\mathrm{Y}$ ? \\
\hline & Does therapeutic procedure $\mathrm{X}$ affect physiologic function $\mathrm{Y}$ ? \\
\hline & Is treatment $\mathrm{X} 1$ more effective than treatment $\mathrm{X} 2 \ldots \mathrm{Xn}$ ? \\
\hline & How should I treat condition X (not limited to drug treatment)? \\
\hline & $\begin{array}{l}\text { What other tests are important to know when ordering/reviewing the medica- } \\
\text { tion/report X on this patient? }\end{array}$ \\
\hline & How should I manage condition X (not specifying diagnostic or therapeutic)? \\
\hline & How is this done at this institution? \\
\hline \multirow{10}{*}{ Medication } & What is the drug of choice for condition $\mathrm{X}$ ? \\
\hline & Does drug $\mathrm{X}$ treat condition $\mathrm{Y}$ ? \\
\hline & $\begin{array}{l}\text { Is drug } \mathrm{X} \text { more effective than drug } \mathrm{X} 1 \ldots \mathrm{Xn} \text { for condition } \mathrm{Y} \text { caused by organism } \\
\mathrm{Z} \text { ? }\end{array}$ \\
\hline & What are the side effects of drug X? \\
\hline & Can drug X cause (adverse finding) Y? \\
\hline & What is the dose of drug $\mathrm{X}$ ? \\
\hline & Are there any interactions between drug X1 and drug X2? \\
\hline & Is low dose of drug $\mathrm{X}$ as effective as high dose of drug $\mathrm{X}$ ? \\
\hline & When should drug X be stopped/started? \\
\hline & Is this the correct preparation of medication $\mathrm{X}$ for this patient? \\
\hline \multirow{9}{*}{ Diagnosis } & What are the causes of condition Y? \\
\hline & What is the cause of symptom $\mathrm{X}$ ? \\
\hline & What is the cause of physical finding $\mathrm{X}$ ? \\
\hline & What is the cause of test finding $\mathrm{X}$ ? \\
\hline & Does drug $\mathrm{X}$ (or therapeutic procedure $\mathrm{X}$ ) cause condition $\mathrm{Y}$ ? \\
\hline & Could this patient have condition X? \\
\hline & What test is indicated in situation $\mathrm{X}$ ? \\
\hline & What is the normal range of test result $\mathrm{X}$ ? \\
\hline & What is normal in this situation? \\
\hline
\end{tabular}


Table 3.3: Most common generic question patterns of physicians' information needs $[1,14,26,27,77]$ (end).

\begin{tabular}{|c|c|}
\hline Questions & \\
\hline \multirow{3}{*}{ Diagnosis (continued) } & Is condition Y1 associated with condition Y2? \\
\hline & Is behavior $\mathrm{W}$ associated with condition $\mathrm{Y} 2 ?$ \\
\hline & $\begin{array}{l}\text { What is the differential diagnosis of sign/symptom/laboratory test/report find- } \\
\text { ing } \mathrm{X} \text { in this patient? }\end{array}$ \\
\hline \multirow{3}{*}{ Prognosis } & What is the prognosis of condition Y? \\
\hline & What is the prognosis of condition Y1 associated with Y2? \\
\hline & What is the prognosis of condition Y complicated by therapeutic procedure $\mathrm{X}$ ? \\
\hline \multirow{2}{*}{ Etiology } & Does organism $\mathrm{Z}$ cause condition $\mathrm{Y}$ ? \\
\hline & Does condition Y affect body substance X? \\
\hline \multirow{2}{*}{ Patient's data } & What are my patient's data? \\
\hline & What inpatient or outpatient medication is this patient on or has been given? \\
\hline \multirow{3}{*}{ Others } & What patient educational information is available about the test/disease $\mathrm{X}$ ? \\
\hline & What is the telephone number/beeper number for attending/resident $\mathrm{X}$ ? \\
\hline & What is this (unfamiliar) domain-specific information? \\
\hline \multirow{5}{*}{ Handling of the CIS } & What does the term/number/abbreviation $\mathrm{X}$ in the CIS mean? \\
\hline & What is the definition of term $\mathrm{X}$ ? \\
\hline & How do you calculate finding $\mathrm{X}$ for this patient with these results? \\
\hline & How do I find the normal value/trend for test $\mathrm{X}$ ? \\
\hline & How do you perform procedure $\mathrm{X}$ to obtain laboratory test $\mathrm{Y}$ ? \\
\hline
\end{tabular}

Due to a lack of data and the heterogeneous categorizations of information of the different studies, the frequency of information categories can only be a rough estimate. Thus, a tentative conclusion is offered instead; the information categories are listed according to their priorities:

1. Information about treatment (e.g., recommended treatment, specific procedures, effects and side effects)

Of particular importance: Information on medication (e.g., recommended medication, effects and side effects, efficiency)

2. Information relevant for diagnosis (e.g., possible causes of symptoms, relations between findings, indicated tests)

3. Information on patient data (e.g., symptoms, medical history, test results)

4. Information about other healthcare providers (e.g., contact data of colleagues and other specialties) 
5. Information about a specific disease (e.g., prognosis, etiology)

The majority of questions posed by physicians deals with therapy and especially with all sorts of aspects associated with medication. The physicians' main interest seems to be the correct drug, its effectiveness, its side effects, dosage, and how long it should be taken. Diagnostic questions also appear quite frequently, but questions concerning the prognosis, the etiology of disease, etc. seem to be of comparatively little concern, if we are to believe the findings in Table 3.3.

\subsubsection{Nurses' Information Needs}

Table 3.4 deals with the information needs of nurse practitioners and nurses in the same manner as the tables concerning the physicians do. It is interesting to note that there is little difference between the questions posed by nurses and what the physicians want to know. The only real difference seems to be their respective focus of attention: for instance, the nurse-patient relationship being of a more personal nature is reflected by the nurses' interest in psycho-social support of patients whereas physicians have not formulated such questions in the studies investigated in this paper.

Table 3.4: Unification of nurses' information needs (NPs: nurse practitioners) (continued on next page).

\begin{tabular}{|c|c|c|}
\hline Category & 1st Subcategory & 2nd Subcategory \\
\hline \multirow{9}{*}{ Information on patient data } & \multicolumn{2}{|l|}{ Demographics } \\
\hline & \multicolumn{2}{|l|}{ Known allergies/intolerances } \\
\hline & \multicolumn{2}{|l|}{ Dispositions } \\
\hline & \multirow{5}{*}{ Current state } & Symptoms \\
\hline & & Diagnosis \\
\hline & & Treatment \\
\hline & & Medication \\
\hline & & Test results \\
\hline & \multicolumn{2}{|l|}{ List of current providers } \\
\hline \multirow{6}{*}{ Information relevant for diagnosis } & \multirow{4}{*}{ Findings/Conditions } & Possible causes \\
\hline & & Calculation \\
\hline & & $\begin{array}{l}\text { Relations between findings (NPs } \\
\text { only) }\end{array}$ \\
\hline & & Effects of findings (NPs only) \\
\hline & Indicated tests (NPs only) & Normal values of test results \\
\hline & Differential diagnoses (NPs only) & \\
\hline \multirow{5}{*}{$\begin{array}{l}\text { Information about } \\
\text { disease/condition }\end{array}$} & Definition & \\
\hline & Description & \\
\hline & Etiology (NPs only) & \\
\hline & Epidemiology (NPs only) & \\
\hline & Symptoms & \\
\hline
\end{tabular}


Table 3.4: Unification of nurses' information needs (NPs: nurse practitioners) (continued).

\begin{tabular}{|c|c|c|}
\hline Category & 1st Subcategory & 2nd Subcategory \\
\hline \multirow{4}{*}{$\begin{array}{l}\text { Information about } \\
\text { disease/condition (continued) }\end{array}$} & Prognosis (NPs only) & Patient specific prognosis \\
\hline & Risk factors & \\
\hline & Physiology & \\
\hline & How to document condition & \\
\hline \multirow{11}{*}{$\begin{array}{l}\text { Information on management } \\
\text { of disease/condition }\end{array}$} & Therapeutic options & $\begin{array}{l}\text { Recommended treatment for } \\
\text { specific condition }\end{array}$ \\
\hline & \multirow{3}{*}{ Treatment } & Institution specific procedure \\
\hline & & Indicated tests (NPs only) \\
\hline & & Instructions for patient \\
\hline & Risks and benefits & Adverse effects \\
\hline & \multirow{6}{*}{ Medication } & $\begin{array}{l}\text { Recommended medication for } \\
\text { specific condition (NPs only) }\end{array}$ \\
\hline & & Risks and benefits \\
\hline & & Adverse effects \\
\hline & & How to administer drug \\
\hline & & Dosage \\
\hline & & Scheduling \\
\hline Information on prophylaxis & $\begin{array}{l}\text { Prophylaxis of a specific } \\
\text { disease/condition (NPs only) }\end{array}$ & \\
\hline $\begin{array}{l}\text { Information on support for the } \\
\text { patient }\end{array}$ & Psycho-social support & \\
\hline \multirow{2}{*}{ Institution specific information } & Policies and protocols & \\
\hline & Census reports & \\
\hline \multirow{7}{*}{$\begin{array}{l}\text { Information about other } \\
\text { healthcare providers }\end{array}$} & $\begin{array}{l}\text { When to seek further professional } \\
\text { advice }\end{array}$ & \\
\hline & When to refer patients & \\
\hline & \multirow{2}{*}{ Healthcare providers at institution } & On-call current providers \\
\hline & & Contact data \\
\hline & \multirow{3}{*}{ Specialty } & Identification \\
\hline & & Available treatments \\
\hline & & Contact data \\
\hline \multirow{2}{*}{ References } & Treatment & \\
\hline & Disease & \\
\hline
\end{tabular}


The most common generic questions of nurses are listed in Table 3.5.

Table 3.5: The most common generic question patterns relating to nurse practitioners' and nurses' information needs $[14,107]$.

\begin{tabular}{|c|c|}
\hline Question & \\
\hline \multirow{6}{*}{ Treatment } & How is this done at this institution? \\
\hline & What is the protocol or procedure for condition $\mathrm{X}$ ? \\
\hline & What do I assess for X? \\
\hline & How should I manage condition X (not specifying diagnostic or therapeutic)? \\
\hline & How should I treat condition $\mathrm{X}$ (not limited to drug treatment)? \\
\hline & What is normal in this situation? \\
\hline \multirow{3}{*}{ Medication } & What is the dose of drug $\mathrm{X}$ ? \\
\hline & Can drug $\mathrm{X}$ cause adverse finding $\mathrm{Y}$ ? \\
\hline & What is the drug of choice for condition $\mathrm{X}$ ? \\
\hline \multirow{6}{*}{ Diagnosis } & What are the symptoms of $\mathrm{X}$ ? \\
\hline & What is the definition of $\mathrm{X}$ ? \\
\hline & What are the risk factors for $\mathrm{X}$ ? \\
\hline & What is the physiology of $\mathrm{X}$ ? \\
\hline & What test is indicated in situation $\mathrm{X}$ ? \\
\hline & What is the cause of test finding $\mathrm{X}$ ? \\
\hline \multirow{2}{*}{ Patient's data } & What are my patient's data? \\
\hline & What are the lab values for $\mathrm{X}$ ? \\
\hline \multirow{3}{*}{ Others } & What is the patient education related to $\mathrm{X}$ ? \\
\hline & What is this (unfamiliar) domain-specific information? \\
\hline & What do I need to document for $\mathrm{X}$ ? \\
\hline
\end{tabular}

Since nurses and nurse practitioners' differ in competence, we list their categories of major interest separately. These are for nurses (sorted according to their priority):

1. Information about treatment

Of particular importance: Information about institution specific procedures, and Information on medication.

2. Information on patient data (e.g., findings, test results, allergies).

3. Educational material and information on instructions for the patient.

4. Information about a specific disease (e.g., description, symptoms, risk factors).

5. Information about other healthcare providers (e.g., contact data, when to seek advice, information about specialties).

6. Information relevant for diagnosis (e.g., possible causes of findings, calculation of findings). 
On the other hand, nurse practitioners require:

1. Information about treatment

Of particular importance: Information on medication.

2. Information relevant for diagnosis (e.g., indicated test, differential diagnoses, relations between findings).

3. Information on patient data (e.g., findings, test results, allergies).

4. Information about a specific disease (e.g., etiology, epidemiology, prognosis).

5. Information about other healthcare providers (e.g., contact data, when to refer patients, information about specialties).

6. Information on psycho-social support for patients.

7. Educational material and information on instructions for the patient.

These lists show the different priorities of nurses and nurse practitioners. The main difference is that nurse practitioners are more concerned with diagnostic issues than nurses. In accordance with the results for physicians, nurses and nurse practitioners need most information about treatment and diagnosis, in which they require information about institution specific procedures and medication quite frequently. In contrast to the results for physicians, there is an obvious need for information on psycho-social support for patients.

\subsubsection{Patients' Information Needs}

Table 3.6 deals with the information needs of patients in the same manner as the tables concerning the physicians and nurses do. The information desired by patients is more voluminous than either the questions formulated by physicians or those formulated by nurses, since they are not only worried about illness itself but also about the effects this illness has on family members, precise information on circumstances they are likely to expect when visiting other medical facilities, and, last but not least, detailed information about available psycho-social support.

Table 3.6: Unification of patients' information needs (continued on next page).

\begin{tabular}{|c|c|c|}
\hline Category & 1st Subcategory & 2nd Subcategory \\
\hline \multirow{5}{*}{ Information on patient data } & Symptoms & \\
\hline & Diagnosis & \\
\hline & Responses to treatment & \\
\hline & Test results & \\
\hline & List of current providers & \\
\hline
\end{tabular}


Table 3.6: Unification of patients' information needs (continued on next page).

\begin{tabular}{|c|c|c|}
\hline Category & 1st Subcategory & 2nd Subcategory \\
\hline \multirow{5}{*}{ Information relevant for diagnosis } & \multirow{3}{*}{ Findings/Conditions } & Possible causes \\
\hline & & Relations between findings \\
\hline & & Effects \\
\hline & \multirow{2}{*}{ Indicated tests } & Purpose \\
\hline & & Expected outcome \\
\hline \multirow{7}{*}{$\begin{array}{l}\text { Information about } \\
\text { disease/condition }\end{array}$} & Definition & \\
\hline & Description & \\
\hline & General prognosis & \\
\hline & \multirow{3}{*}{ Patient specific prognosis } & Chances of cure \\
\hline & & $\begin{array}{l}\text { Diagnostic stage / extent of } \\
\text { involvement }\end{array}$ \\
\hline & & Progression \\
\hline & \multicolumn{2}{|l|}{ Possible effects } \\
\hline \multirow{26}{*}{$\begin{array}{l}\text { Information on management } \\
\text { of disease/condition }\end{array}$} & \multirow{2}{*}{ Therapeutic options } & All treatment options \\
\hline & & Available treatment options \\
\hline & \multirow{6}{*}{ Treatment } & Institution specific procedure \\
\hline & & Scheduling \\
\hline & & Interventions \\
\hline & & Medication \\
\hline & & Indicated tests \\
\hline & & Instructions for patient \\
\hline & \multirow{8}{*}{ Risks and benefits } & Intended effects \\
\hline & & Adverse effects \\
\hline & & Physical effects \\
\hline & & Emotional effects \\
\hline & & Effects on attractiveness \\
\hline & & Effects on risk of further disease \\
\hline & & Effects on quality of life \\
\hline & & Possible complications \\
\hline & \multirow{2}{*}{ Description } & How it works \\
\hline & & At home / at hospital \\
\hline & \multicolumn{2}{|l|}{ Expected progress } \\
\hline & \multicolumn{2}{|l|}{ Expected outcome } \\
\hline & \multicolumn{2}{|l|}{ Care following procedures } \\
\hline & \multicolumn{2}{|l|}{ Length of recovery } \\
\hline & \multirow{4}{*}{ Medication } & Risks and benefits \\
\hline & & How to take it \\
\hline & & Dosage \\
\hline & & Scheduling \\
\hline
\end{tabular}


Table 3.6: Unification of patients' information needs (continued on next page).

\begin{tabular}{|c|c|c|}
\hline Category & 1st Subcategory & 2nd Subcategory \\
\hline \multirow{15}{*}{ Instructions for patient } & \multirow{9}{*}{ Patient's restrictions } & Healthy eating \\
\hline & & Diet \\
\hline & & Alcohol \\
\hline & & Driving \\
\hline & & Exertion/Sports \\
\hline & & Rest time \\
\hline & & Traveling \\
\hline & & Hobbies \\
\hline & & Bathing \\
\hline & \multicolumn{2}{|l|}{ Pain relief } \\
\hline & \multirow{2}{*}{ Recovery } & How to speed up recovery \\
\hline & & Options for rehabilitation \\
\hline & \multirow{2}{*}{ Preparation } & Preparation for treatment \\
\hline & & Preparing information for carers \\
\hline & Follow-up arrangements & \\
\hline \multirow{3}{*}{ Information on prophylaxis } & \multirow{2}{*}{ Of a specific disease/condition } & Recommended actions \\
\hline & & Medication \\
\hline & $\begin{array}{l}\text { Preventing recurrent or subsequent } \\
\text { disease }\end{array}$ & \\
\hline \multirow{8}{*}{$\begin{array}{l}\text { Information on support } \\
\text { for the patient }\end{array}$} & \multirow{6}{*}{ Psycho-social support } & Self-help groups \\
\hline & & Voluntary organizations \\
\hline & & Other peoples' experiences \\
\hline & & Social workers \\
\hline & & Mental health workers \\
\hline & & Available benefits \\
\hline & \multirow{2}{*}{ Physical support } & Gadgets \\
\hline & & Local health services \\
\hline \multirow{3}{*}{$\begin{array}{l}\text { Information about impact } \\
\text { on family members }\end{array}$} & Roles of family members & \\
\hline & Risk for family members & \\
\hline & $\begin{array}{l}\text { How to handle physical and } \\
\text { emotional impact }\end{array}$ & \\
\hline \multirow[t]{2}{*}{$\begin{array}{l}\text { Information about other } \\
\text { healthcare providers }\end{array}$} & $\begin{array}{l}\text { When to seek further professional } \\
\text { advice }\end{array}$ & \\
\hline & Contact data & \\
\hline
\end{tabular}


Table 3.6: Unification of patients' information needs (end).

\begin{tabular}{|c|c|c|}
\hline Category & 1st Subcategory & 2nd Subcategory \\
\hline \multirow{9}{*}{$\begin{array}{l}\text { Information about other } \\
\text { healthcare providers (continued) }\end{array}$} & \multirow{9}{*}{ Other specialties } & Description \\
\hline & & Available treatments \\
\hline & & Hours \\
\hline & & Contact data (in and out of hours) \\
\hline & & Length of visits \\
\hline & & Parking \\
\hline & & Staff available to help \\
\hline & & Facilities \\
\hline & & $\begin{array}{l}\text { Going home after visits (driving } \\
\text { etc.) }\end{array}$ \\
\hline \multirow{7}{*}{ References } & Treatment & \\
\hline & Disease & \\
\hline & Services & \\
\hline & New research & \\
\hline & Relevant books & \\
\hline & $\begin{array}{l}\text { Areas of scientific or medical } \\
\text { uncertainties }\end{array}$ & \\
\hline & Contact data & \\
\hline
\end{tabular}

Only one of the reviewed studies about patients' information needs provides a list of frequently asked generic questions (see Table 2.12).

The most important categories of information for patients are (sorted according to priority):

1. Information about treatment (e.g., therapeutic options, risks and benefits, therapeutic procedures).

Of particular importance: Information on effects of the treatment (e.g., on quality of life, physical and emotional effects).

2. Information about their disease

Of particular importance: Information on individual prognosis,

Chances of recovery, and

Information on their diagnostic stage.

3. Information on available psycho-social support (e.g., other peoples' experiences, mental health workers, voluntary organizations).

4. Information regarding their family (e.g., risks for family members, and how to handle physical and emotional impact on family members).

5. Their data (e.g., test findings).. 
6. Prevention of specific disease or recurrent illness.

7. Educational material and instructions (e.g., information on treatment and disease, diet, exertion, pain relief, preparation for treatment).

It is evident from our results, that patients' information needs differ qualitatively from physicians' and nurses' information needs. They require much more detailed information on emotional effects of their treatment and its effects on their quality of life. As is to be expected, that prognosis and chances of recovery are also of major interest for patients, as is available support. Moreover, in contrast to the results for physicians and nurses, patients require detailed information about any effects their illness may have on family members.

Finally, we have normalized and merged the findings from all studies on clinical information needs, regarding physicians, nurses, and patients. This has resulted in a comprehensive categorization of clinical information needs as given in the following section.

\subsubsection{Categorization of Clinical Information Needs}

For the software modeler to be able to design a practical software tool, there has to be a complete catalogue of every single aspect that may be related to information needs of any party concerned. It may not be necessary and probably not always be possible to include all of the following points in such a program, but the decision which points to include and which to leaf out can only be made on the basis of such an all-comprising list as given in Figure 3.2 to Figure 3.11.

\subsection{Conclusion}

In this thesis we have investigated the different user groups of a computerized clinical guideline, the process of formalizing such a guideline, as well as the clinical information needs that have to be integrated when executing the guideline. Based on the findings of a comprehensive literature research, we have focused on the information requirements of these four user groups: guideline modelers, physicians, nurses, and patients.

We found several studies about the information needs in clinical care, e.g., surveys on the information needs of physicians $[1,12,14,26,27,36,56,77,83,108]$, of the nursing staff $[7,13,14$, $55,56,106,107]$, and of patients $[15,23,45,49,59,71,76,87,88,102]$. Due to a lack of useful surveys on the information needs of guideline modelers, we have examined the modelers' tasks involved in the development of a computerized clinical guideline $[6,10,21,46,47,60,67,68,72,78,82,86$, 97, 100, 101, 103].

From these studies, we have derived specific needs for each user group:

- We give an overview of tasks that are important in transforming a textual CPG into a guideline representation model, including the identification of knowledge elements and making them independent from the surrounding text, the modeling of clinical actions, and the generation of vocabularies.

- Moreover, we give a comprehensive list of information needs of physicians; above all physicians seek information about treatment and medication.

- We give a comprehensive list of information needs of nurses; besides information about treatment and medication, they show an obvious need for information on psycho-social support for patients. 


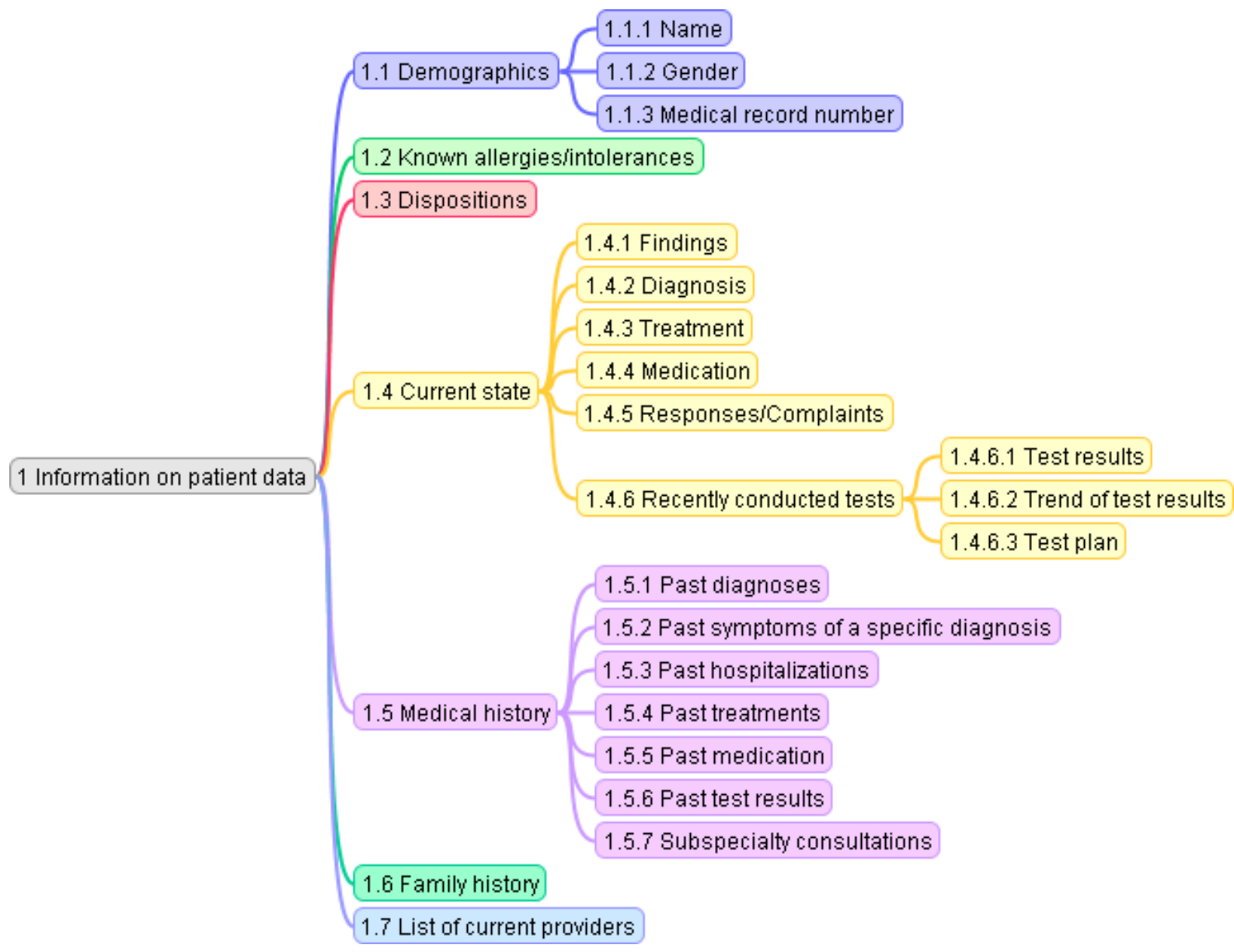

Figure 3.2: Information on patient data.

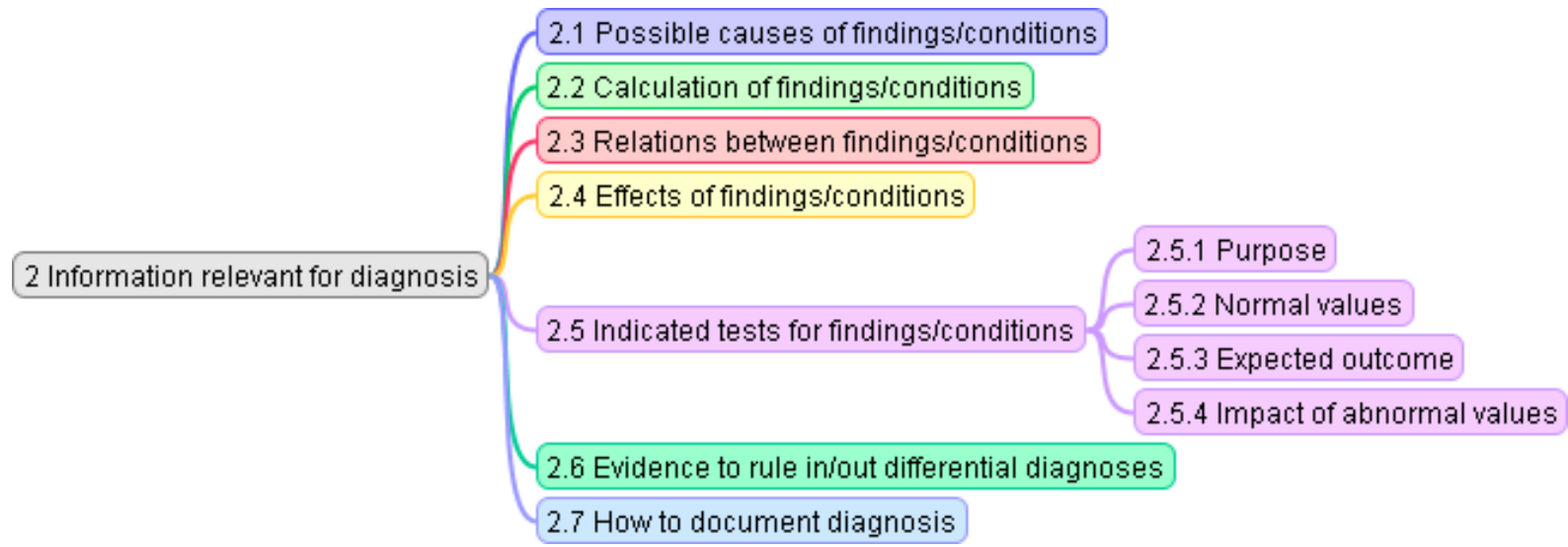

Figure 3.3: Information relevant for diagnosis.

- Last but not least, we give a detailed outline of information that should be provided for patients, for instance, they require detailed information about effects on their quality of life, their specific prognosis, and about chances of recovery.

Subsequently, we have categorized, merged, and presented these needs in a concise, comprehensible way. By providing categorized information from several studies and publications, 


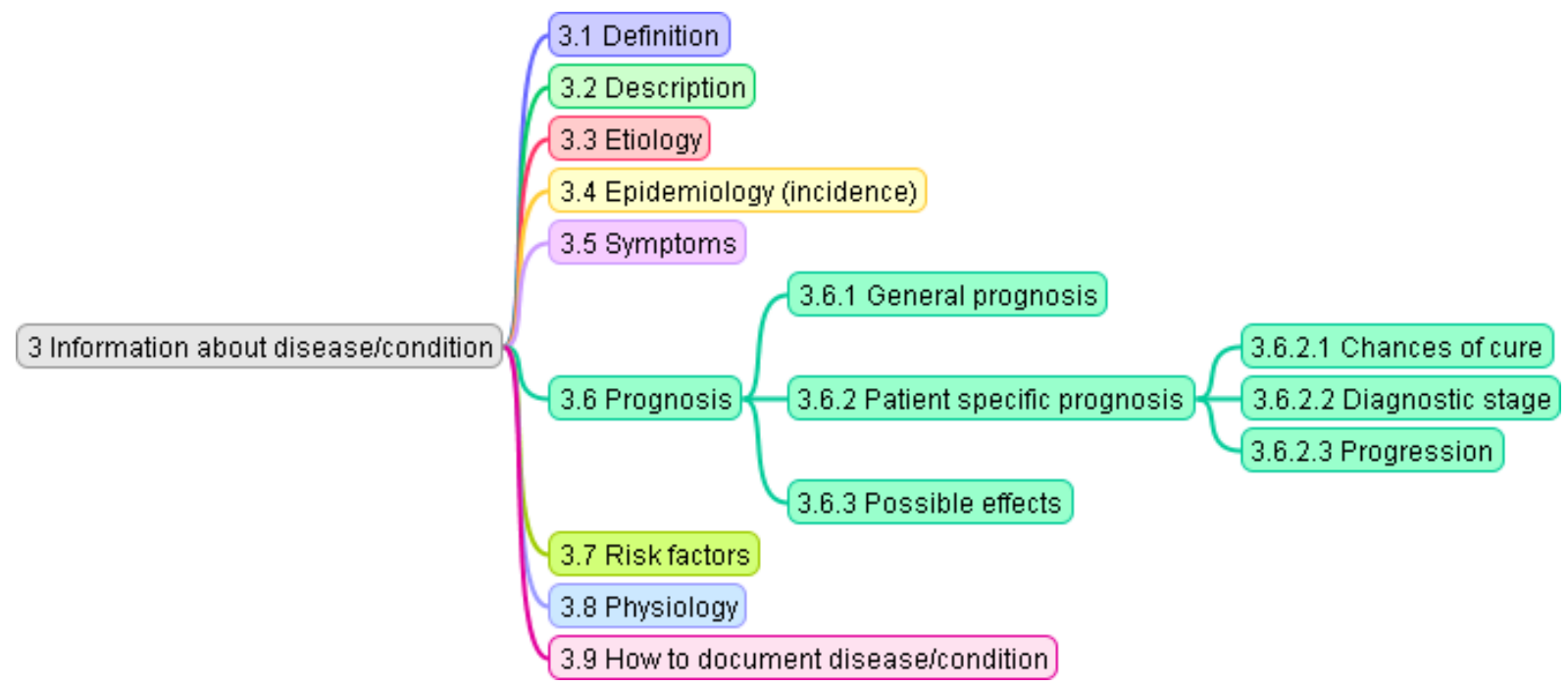

Figure 3.4: Information about a disease/condition.

we establish an information basis crucial to the design of a useful software tool facilitating the formalization and the execution of a CPG. Which of these tasks and information needs can be supported by a software tool depends on the potential and purpose of the individual tool. However, it is important for developers to carefully consider every single aspect presented in this thesis, since all of them have been formulated as modeling tasks or as actual information needs in clinical praxis. 


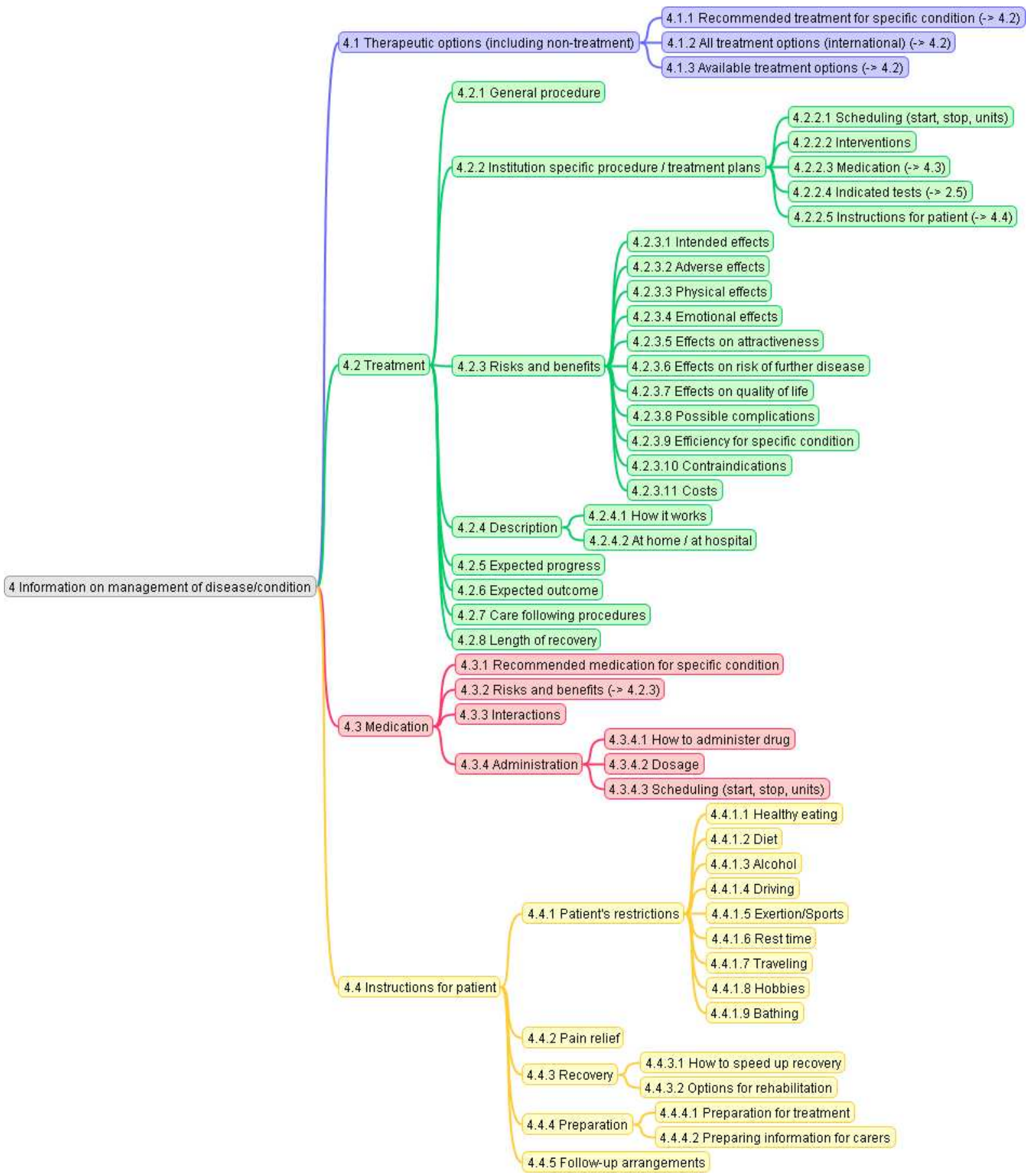

Figure 3.5: Information on the management of the disease/condition.

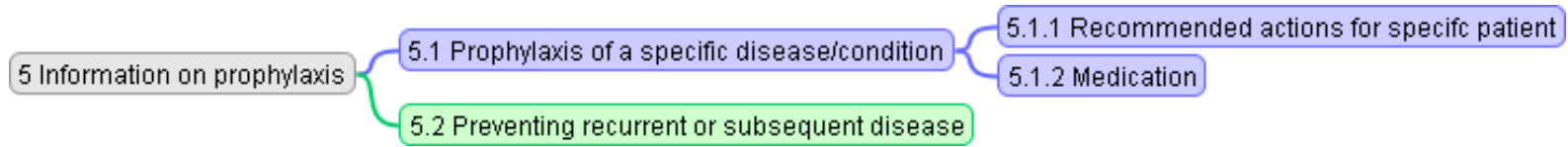

Figure 3.6: Information on prophylaxis. 


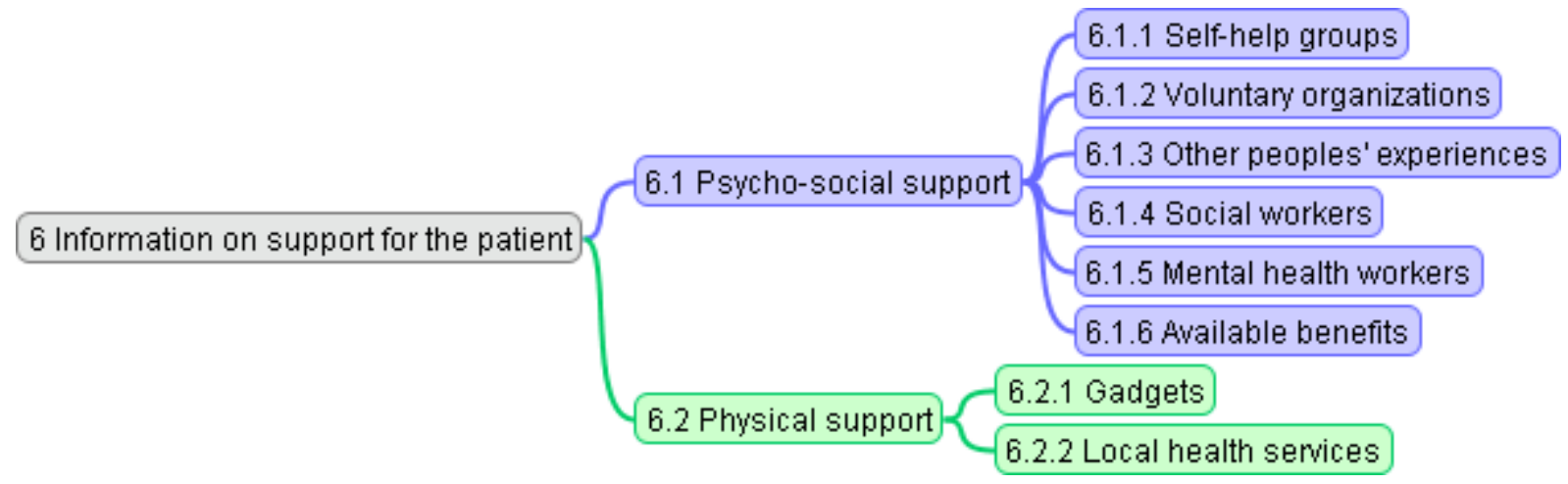

Figure 3.7: Information on support for the patient.

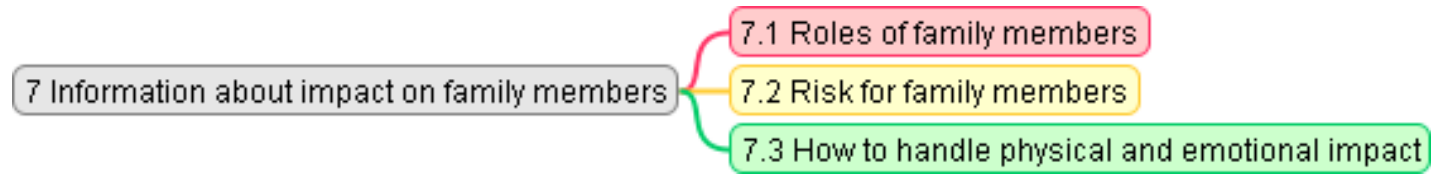

Figure 3.8: Information about the impact of the condition on family members.

8 Institution specific information

8.1 Policies and protocols

8.2 Census reports

Figure 3.9: Institution specific information.

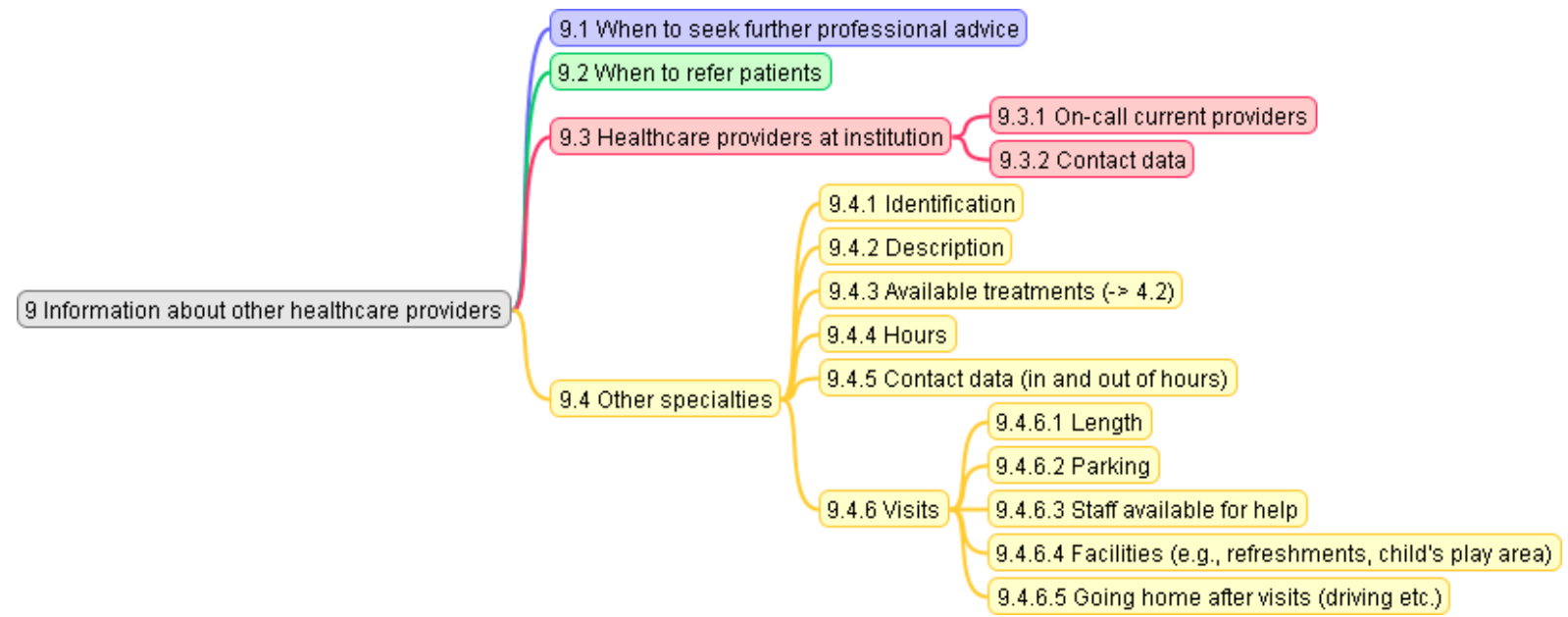

Figure 3.10: Information about other healthcare providers. 


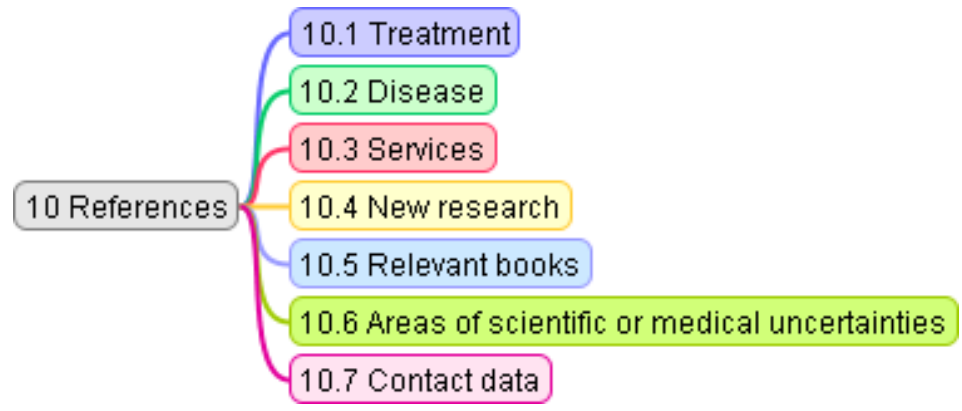

Figure 3.11: References to important information. 


\section{Appendix A}

\section{Detailed Results of Studies}

\section{A.1 Results of Studies on Physicians' Information Needs}

Table A.1: Most common generic questions derived from two studies in 1999 (103 family doctors) and 2000 (49 primary care doctors) by Ely et al. [26,27].

\begin{tabular}{ll}
\hline Questions & Frequency \\
\hline What is the drug of choice for condition X? & $11 \%$ \\
\hline What is the cause of symptom X? & $8 \%$ \\
\hline What test is indicated in situation X? & $8 \%$ \\
\hline What is the dose of drug X? & $7 \%$ \\
\hline How should I treat condition X (not limited to drug treatment)? & $6 \%$ \\
\hline How should I manage condition X (not specifying diagnostic or therapeutic)? & $5 \%$ \\
\hline What is the cause of physical finding X? & $5 \%$ \\
\hline What is the cause of test finding X? & $5 \%$ \\
\hline Can drug X cause (adverse finding) Y? & $4 \%$ \\
\hline Could this patient have condition X? & $4 \%$ \\
\hline
\end{tabular}


Table A.2: Information needs and communication difficulties perceived by physicians (McKnight et al., $2002[56])$.

\begin{tabular}{ll}
\hline Field & Information Need \\
\hline & A list of current medication and time administered \\
& Problem list \\
Patient Specific & Outpatient notes (especially subspecialty consultations) \\
& A central list of current providers for the patient (consultants, \\
& nurses) \\
& Laboratory and other test results \\
\hline Institution Specific & Current providers that are on-call and how to contact them \\
\hline \multirow{2}{*}{ Domain Specific } & Disease management information \\
& Prescribing information \\
& Medical formulas linked to patient data \\
\hline Communication Difficulties & $\begin{array}{l}\text { Identifying and contacting other health care providers (especially } \\
\text { consultation services) }\end{array}$ \\
\hline
\end{tabular}

Table A.3: Frequency of generic question types asked by 6 medical residents (and average number by session) as described by Collins et al., 2009 [14].

\begin{tabular}{lrr}
\hline Generic question type & Frequency & Average per session \\
\hline What is the dose of drug X? & 33 & 5.5 \\
\hline How is this done at this institution? & 29 & 4.8 \\
\hline What is this (unfamiliar) domain-specific information? & 22 & 3.7 \\
\hline Can drug X cause adverse finding Y? & 14 & 2.3 \\
\hline What are my patient's data? & 10 & 1.7 \\
\hline What is normal in this situation? & 6 & 1.0 \\
\hline How should I treat condition X?+ & 5 & 0.8 \\
\hline What is the drug of choice for condition X? & 4 & 0.7 \\
\hline How should I manage condition X?* & 3 & 0.5 \\
\hline What test is indicated in situation X? & 1 & 0.2 \\
\hline What is the patient education related to X? & 0 & 0.0 \\
\hline What is the cause of test finding X? & 0 & 0.0 \\
\hline Total & $\mathbf{1 2 7}$ & $\mathbf{2 1 . 2}$ \\
\hline
\end{tabular}

*not specifying diagnostic or therapeutic

${ }^{+}$not limited to drug treatment 


\section{A.2 Results of Studies on Nurses' Information Needs}

Table A.4: Results of 134 questionnaires about the frequency of information needs of nurse practitioners' in North Carolina (Cogdill, 2003 [13]).

\begin{tabular}{lll}
\hline Need & $\begin{array}{l}\text { Weekly } \\
\text { frequency }\end{array}$ & $\begin{array}{l}\text { Frequency } \\
\text { per patient }\end{array}$ \\
\hline Drug therapy & 8.6 & 0.21 \\
\hline Diagnosis & 5.8 & 0.15 \\
\hline Other therapy & 5.4 & 0.13 \\
\hline Referral & 3.1 & 0.08 \\
\hline Etiology & 3.0 & 0.08 \\
\hline Psychosocial & 2.6 & 0.09 \\
\hline Disposition & 2.4 & 0.06 \\
\hline Epidemiology & 2.1 & 0.05 \\
\hline
\end{tabular}

Frequency per patient was obtained by dividing weekly frequency by the reported number of patients per week. The category of disposition refers to information needs about where to send a patient (not including the referral of the patient to another healthcare provider). 
Table A.5: Results of 134 questionnaires about the frequency of information resource use by nurse practitioners' in North Carolina (Cogdill, 2003 [13]).

\begin{tabular}{lllllll}
\hline \multicolumn{7}{c}{ Frequency of use } \\
\hline Resource & $\begin{array}{l}\text { A few } \\
\text { times a } \\
\text { week or } \\
\text { more }\end{array}$ & $\begin{array}{l}\text { At least } \\
\text { once a } \\
\text { month }\end{array}$ & $\begin{array}{l}\text { Once } \\
\text { every } \\
\text { few } \\
\text { months }\end{array}$ & $\begin{array}{l}\text { About } \\
\text { once a } \\
\text { year }\end{array}$ & Never & $\begin{array}{l}\text { Not } \\
\text { reported }\end{array}$ \\
\hline $\begin{array}{l}\text { Primary } \\
\text { supervising } \\
\text { physician }\end{array}$ & $84(63 \%)$ & $33(25 \%)$ & $12(9 \%)$ & $0(-)$ & $1(1 \%)$ & $4(3 \%)$ \\
\hline $\begin{array}{l}\text { Drug reference } \\
\text { manual }\end{array}$ & $82(61 \%)$ & $37(28 \%)$ & $11(8 \%)$ & $0(-)$ & $0(-)$ & $4(3 \%)$ \\
\hline Textbook & $68(51 \%)$ & $50(37 \%)$ & $11(8 \%)$ & $1(1 \%)$ & $0(-)$ & $4(3 \%)$ \\
\hline Journal article & $40(30 \%)$ & $62(46 \%)$ & $24(18 \%)$ & $4(3 \%)$ & $0(-)$ & $4(3 \%)$ \\
\hline $\begin{array}{l}\text { Other nurse } \\
\text { practitioner }\end{array}$ & $35(26 \%)$ & $24(18 \%)$ & $37(28 \%)$ & $26(19 \%)$ & $8(6 \%)$ & $4(3 \%)$ \\
\hline Other physician & $33(25 \%)$ & $45(34 \%)$ & $37(28 \%)$ & $12(9 \%)$ & $3(2 \%)$ & $4(3 \%)$ \\
\hline Pharmacist & $19(14 \%)$ & $55(41 \%)$ & $39(29 \%)$ & $15(11 \%)$ & $0(-)$ & $6(5 \%)$ \\
\hline
\end{tabular}

Table A.6: Frequency of information needs resulting from interviews of 20 nurse practitioners in North Carolina after their encounters with patients (Cogdill, 2003 [13]).

\begin{tabular}{lll}
\hline Category & $\begin{array}{l}\text { Frequency of } \\
\text { information needs }\end{array}$ & $\begin{array}{l}\text { Number of participants } \\
\text { reporting need }\end{array}$ \\
\hline Drug therapy & $32(43 \%)$ & $14(70 \%)$ \\
\hline Diagnosis & $31(41 \%)$ & $16(80 \%)$ \\
\hline General management & $4(5 \%)$ & $3(15 \%)$ \\
\hline Other therapy & $2(3 \%)$ & $2(10 \%)$ \\
\hline Referral & $2(3 \%)$ & $2(10 \%)$ \\
\hline Psychosocial & $2(3 \%)$ & $2(10 \%)$ \\
\hline Prognosis & $2(3 \%)$ & $2(10 \%)$ \\
\hline
\end{tabular}


Table A.7: Frequency of generic question types asked by 4 nurse practitioners and 3 registered nurses from the New York Presbyterian Hospital while using a clinical information system (Collins et al., 2009 [14]).

\begin{tabular}{|c|c|c|c|}
\hline \multirow[b]{2}{*}{ Generic question type } & \multicolumn{3}{|c|}{ Frequency (average per session) } \\
\hline & Nurse practitioners & Registered nurses & Total \\
\hline How is this done at this institution? & $20(5.0)$ & $8(2.7)$ & $28(4.0)$ \\
\hline What is the patient education related to $\mathrm{X}$ ? & $1(0.3)$ & $23(7.7)$ & $24(3.4)$ \\
\hline Can drug $\mathrm{X}$ cause adverse finding $\mathrm{Y}$ ? & $13(3.3)$ & $6(2.0)$ & $19(2.7)$ \\
\hline What are my patient's data? & $6(1.5)$ & $12(4.0)$ & $18(2.6)$ \\
\hline How should I manage condition X?* & $5(1.3)$ & $8(2.7)$ & $13(0.4)$ \\
\hline What is the dose of drug $\mathrm{X}$ ? & $10(2.5)$ & $1(0.3)$ & $11(1.6)$ \\
\hline $\begin{array}{l}\text { What is this (unfamiliar) domain-specific } \\
\text { information? }\end{array}$ & $3(0.8)$ & $6(2.0)$ & $9(1.3)$ \\
\hline How should I treat condition $\mathrm{X} ?^{+}$ & $2(0.5)$ & $6(2.0)$ & $8(1.1)$ \\
\hline What is normal in this situation? & $4(1.0)$ & $1(0.3)$ & $5(0.7)$ \\
\hline What is the drug of choice for condition X? & $3(0.8)$ & $0(0.0)$ & $3(0.4)$ \\
\hline What test is indicated in situation $\mathrm{X}$ ? & $1(0.3)$ & $0(0.0)$ & $1(0.1)$ \\
\hline What is the cause of test finding $\mathrm{X}$ ? & $1(0.3)$ & $0(0.0)$ & $1(0.1)$ \\
\hline Total per clinician type & $69(17.3)$ & $71(23.7)$ & $140(20.0)$ \\
\hline
\end{tabular}

$*_{\text {not specifying diagnostic or therapeutic }}$

${ }^{+}$not limited to drug treatment 


\section{A.3 Results of Studies on Patients' Information Needs}

Richardson and Moran, 1995 The standard condition-specific patient information according to Richardson and Moran is thought to include [71]:

- Simple description of condition (a separate leaflet about operations can be useful)

- Brief overview of treatment options including non-treatment

- Benefits of treatment (impact on quality of life)

- Risks and side effects of treatment (impact on quality of life)

- Clear and unambiguous statements regarding preparation for specific procedures

- Description of care following procedures

- Dos and Don'ts on going home, including advice, as appropriate, about: rest, time off work, everyday activity, diet or feeding, pain relief, alcohol, bowels or toileting, driving, lifting, bathing, certification

- Length of recovery phase and how the patient may feel at each stage to full recovery

- Information on getting results of tests, as appropriate

- Follow-up arrangements, as appropriate

- Contact name and telephone number

- References (if known) and contact addresses for more detailed information (if appropriate)

Furthermore, they recommend to include the following information into the specialty-specific patient information [71]:

- Simple description of the specialty and range of treatments available

- Clear directions to reach the specialty, written and pictorial, including possibilities for parking

- Staff who are available to help

- How specialty can benefit the patient

- How and when specialty can be accessed by patients (and relatives)

- Clear instructions about any specific pre-attendance preparations

- Clear statement about facilities (e.g. refreshments, child's play area)

- What to expect during outpatient attendance (e.g. length of visit, need for tests)

- Information about going home after visit (e.g. driving)

- Contact names and telephone numbers for help in and out of hours

- When to seek further professional advice

- Details about results and follow-up arrangements 
Table A.8: Answers of 250 cancer patients in west Scotland to specific questions about their need for information (Meredith et al., 1996 [59]).

\begin{tabular}{llll}
\hline Question & $\begin{array}{l}\text { Do not } \\
\text { to know }\end{array}$ & $\begin{array}{l}\text { Would like } \\
\text { know }\end{array}$ & $\begin{array}{l}\text { Absolute need } \\
\text { to know }\end{array}$ \\
\hline Whether illness is cancer & $4 \%$ & $24 \%$ & $72 \%$ \\
\hline What is specific medical name of illness & $25 \%$ & $46 \%$ & $30 \%$ \\
\hline What is week by week progress & $10 \%$ & $42 \%$ & $48 \%$ \\
\hline What are chances of cure & $9 \%$ & $31 \%$ & $60 \%$ \\
\hline What are all possible treatments & $14 \%$ & $32 \%$ & $54 \%$ \\
\hline What are all possible side effects of treatment & $6 \%$ & $21 \%$ & $73 \%$ \\
\hline Exactly how treatment works to treat illness & $20 \%$ & $36 \%$ & $43 \%$ \\
\hline
\end{tabular}

Kerr et al., 2005 Kerr et al. investigated what patients require from interactive health communication applications for chronic disease [49]:

- What to expect of the condition and treatment, e.g., usual course of the condition, possible complications, tests and treatments that may be offered

- Medication, e.g., indications for use and potential side effects

- The practicalities of day-to-day living, e.g., going on holiday, traveling, planning what food to buy and eat

- Practical information, e.g., information about legal issues, benefits available, where to buy relevant books and gadgets

- Local services and resources, e.g., local health services, voluntary organizations and self-help groups

- Information about available treatments, in the UK and treatments available elsewhere

- In-depth scientific information, about the condition and treatments

- Information about new research, but with a warning if these findings have not been confirmed or generally accepted

- Information about areas of scientific or medical uncertainty, with an explanation of what this uncertainty means for users

- Other people's experiences, e.g., personal stories from other people with similar health problems

- Information for family members, addressing the concerns and roles of those around the patients 
Table A.9: Seven most popular sections of cancer information booklets chosen by 65 breast cancer patients (Jones et al., $2006[45]$ ).

\begin{tabular}{lr}
\hline Information Section (Booklet) & \% of Patients \\
\hline Healthy eating (Diet and the Cancer Patient) & $35 \%$ \\
\hline Possible causes (Understanding Breast Cancer) & $32 \%$ \\
\hline Side effects (Understanding Radiotherapy) & $29 \%$ \\
\hline General tips (Understanding Radiotherapy) & $29 \%$ \\
\hline Why prescribed (Understanding Radiotherapy) & $28 \%$ \\
\hline Living with surgery (Understanding Breast Cancer) & $26 \%$ \\
\hline Feelings (Cancer and Complementary Therapies) & $26 \%$ \\
\hline
\end{tabular}

Table A.10: Seven most popular sections of cancer information booklets chosen by 29 prostate cancer patients (Jones et al., $2006[45]$ ).

\begin{tabular}{lr}
\hline Information Section (Booklet) & \% of Patients \\
\hline Side effects (Understanding Radiotherapy) & $45 \%$ \\
\hline Radiotherapy (Understanding Prostate Cancer) & $34 \%$ \\
\hline Why prescribed (Understanding Radiotherapy) & $34 \%$ \\
\hline Healthy eating (Diet and the Cancer Patient) & $31 \%$ \\
\hline Prostate cancer (Understanding Prostate Cancer) & $28 \%$ \\
\hline Possible causes (Understanding Prostate Cancer) & $28 \%$ \\
\hline General tips (Understanding Radiotherapy) & $24 \%$ \\
\hline
\end{tabular}




\section{Bibliography}

[1] M. Allen, L. Currie, M. Graham, S. Bakken, V. L. Patel, and J. J. Cimino. The classification of clinicians' information needs while using a clinical information system. In Proceedings of the American Medical Informatics Association Annual Fall Symposium, pages 26-30, 2003.

[2] American Medical Informatics Association. AMIA - home page. http://www.amia.org/ (last accessed: Sep 22, 2009).

[3] Artificial Intelligence in Medicine. AIIM - home page. http://www.aiimjournal.com/ (last accessed: Sep 22, 2009).

[4] Audit Commission for Local Authorities and the National Health Service in England and Wales. What seems to be the matter: Communication between hospitals and patients. Audit Commission National Health Service Report; No. 12. HMSO, London, UK, 1993.

[5] M. Balser, O. Coltell, J. van Croonenborg, C. Duelli, F. van Harmelen, P. L. A. Jovell, M. Marcos, S. Miksch, W. Reif, K. Rosenbrand, A. Seyfang, and A. ten Teije. Protocure: Supporting the development of medical protocols through formal methods. In K. Kaiser, S. Miksch, and S. Tu, editors, Computer-based Support for Clinical Guidelines and Protocols. Proceeding of the Symposium on Computerized Guidelines and Protocols, volume 101 of Studies in Health Technology and Informatics, pages 103-107, Prague, Czech Republic, 2004. IOS Press.

[6] N. Beard, J. R. Campbell, S. M. Huff, M. Leon, J. G. Mansfield, E. Mays, J. McClay, D. N. Mohr, M. A. Musen, D. O'Brien, R. A. Rocha, A. Saulovich, S. M. Scheitel, and S. W. Tu. Standards-based sharable active guideline environment (SAGE): A project to develop a universal framework for encoding and disseminating electronic clinical practice guidelines. In Proceedings of the American Medical Informatics Association Annual Fall Symposium, pages 973-978, 2002.

[7] J. Blythe. Assessing nurses' information needs in the work environment. Bulletin of the Medical Library Association, 81(4):433-435, 1993.

[8] V. M. Bowden, M. E. Kromer, and R. C. Tobia. Assessment of physicians' information needs in five Texas counties. Bulletin of the Medical Library Association, 82:189-196, 1994.

[9] British Medical Journal. BMJ - home page. http://www.bmj.com/ (last accessed: Sep 22, 2009).

[10] D. Buchtela, J. Peleška, A. Veselý, J. Zvárová, and M. Zvolský. Formalization of clinical practice guidelines. In eHealth Beyond the Horizon - Get IT There. Proceedings of the 21st International Congress of the European Federation of Medical Informatics (MIE 2008), 
volume 136 of Studies in Health Technology and Informatics, pages 152-156, Göteborg, Sweden, 2008. IOS Press.

[11] D. R. Calkins, R. B. Davis, S. P. Reiley, R. S. Phillips, K. L. C. Pineo, T. L. Delbanco, and L. I. Iezzoni. Patient-physician communication at hospital discharge and patients' understanding of the postdischarge treatment plan. Archives of Internal Medicine, 157(9): 1026-1030, 1997.

[12] M. L. Chambliss and J. Conley. Answering clinical questions. Journal of Family Practice, 43:140-144, 1996.

[13] K. W. Cogdill. Information needs and information seeking in primary care: A study of nurse practitioners. Journal of the Medical Library Association, 91(2):203-215, 2003.

[14] S. A. Collins, L. M. Currie, S. Bakken, and J. J. Cimino. Information needs, Infobutton Manager use, and satisfaction by clinician type: A case study. Journal of the American Medical Informatics Association, 16(1):140-142, 2009.

[15] A. Coulter, V. Entwistle, and D. Gilbert. Sharing decisions with patients: is the information good enough? British Medical Journal, 318:318-322, 1999.

[16] D. G. Covell, G. C. Uman, and P. R. Manning. Information needs in office practice: Are they being met? Annals of Internal Medicine, 103(4):596-599, 1985.

[17] L. M. Currie, M. Graham, M. Allen, S. Bakken, V. Patel, and J. J. Cimino. Clinical information needs in context: An observational study of clinicians while using a clinical information system. In Proceedings of the American Medical Informatics Association Annual Fall Symposium, pages 190-194, 2003.

[18] L. H. Daltroy. Doctor-patient communication in rheumatological disorders. Baillière's Clinical Rheumatology, 7(2):221-239, 1993.

[19] P. A. de Clercq, J. A. Blom, H. H. M. Korsten, and A. Hasman. Approaches for creating computer-interpretable guidelines that facilitate decision support. Artificial Intelligence in Medicine, 31:1-27, 2004.

[20] P. A. de Clercq, A. Hasman, J. A. Blom, and H. H. M. Korsten. Design and implementation of a framework to support the development of clinical guidelines. International Journal of Medical Informatics, 64:285-318, 2001.

[21] P. A. de Clercq, K. Kaiser, and A. Hasman. Computer-interpretable guideline formalisms. chapter 2, pages $22-43$.

[22] L. F. Degner, J. M. Farber, T. F. L. Hack, and M. E. McWilliams. Communication between cancer patients and health care professionals. An annotated bibliography. National Cancer Institute of Canada, Winnipeg, Manitoba, 1989.

[23] D. Dickinson and D. K. T. Raynor. Ask the patients - they may want to know more than you think. British Medical Journal, 327:861, 2003.

[24] P. L. Elkin, M. Peleg, R. Lacson, E. Bernstam, S. W. Tu, A. Boxwala, R. Greenes, and E. H. Shortliffe. Toward standardization of electronic guideline representation. $M D$ Computing, 17(6):39-44, 2000. 
[25] J. W. Ely, R. J. Burch, and D. C. Vinson. The information needs of family physicians: Case specific clinical questions. Journal of Family Practice, 38:265-269, 1992.

[26] J. W. Ely, J. A. Osheroff, M. H. Ebell, G. R. Bergus, B. T. Levy, M. L. Chambliss, and E. R. Evans. Analysis of questions asked by family doctors regarding patient care. British Medical Journal, 319:358-361, 1999.

[27] J. W. Ely, J. A. Osheroff, P. N. Gorman, M. H. Ebell, M. L. Chambliss, E. A. Pifer, and P. Z. Stavri. A taxonomy of generic clinical questions: Classification study. British Medical Journal, 321:429-432, 2000.

[28] A. Essaihi, G. Michel, and R. N. Shiffman. Comprehensive categorization of guideline recommendations: Creating an action palette for implementers. In M. A. Musen, editor, Proceedings of the American Medical Informatics Association Annual Fall Symposium, pages 220-224, Washington, DC, 2003. Hanley and Belfus.

[29] M. J. Field and K. N. Lohr, editors. Clinical Practice Guidelines: Directions for a New Program. National Academies Press, Institute of Medicine, Washington DC, 1990. http://www.nap.edu/books/0309043468/html/index.html (last accessed: July 15, 2009).

[30] M. Fowler and K. Scott. UML Distilled: A Brief Guide to the Standard Object Modeling Language (2nd Edition). Addison-Wesley Professional, Reading, MA, August 1999.

[31] J. Fox, N. Johns, C. Lyons, A. Rahmanzadeh, R. Thomson, and P. Wilson. PROforma: A general technology for clinical decision support systems. Computer Methods and Programs in Biomedicine, 54(1-2):59-67, 1997.

[32] J. Fox, N. Johns, A. Rahmanzadeh, and R. Thomson. PROforma: A method and language for specifying clinical guidelines and protocols. In Proceedings of the Medical Informatics Europe Conference, Amsterdam, 1996.

[33] J. H. Gennari, M. A. Musen, R. W. Fergerson, W. E. Grosso, M. Crubézy, H. Eriksson, N. F. Noy, and S. W. Tu. The evolution of Protégé: An environment for knowledge-based systems development. International Journal of Human Computer Studies, 58(1):89-123, 2002 .

[34] M. A. Gillispie and L. B. M. Ellis. Computer-based patient education revisited. Journal of Medical Systems, 17(3-4):119-125, 1993.

[35] Google Scholar. Search Engine. http://scholar.google.at/ (last accessed: Sep 22, 2009).

[36] M. L. Green, M. A. Ciampi, and P. J. Ellis. Residents' medical information needs in clinic: Are they being met? American Journal of Medicine, 109(3):218-223, 2000.

[37] R. A. Greenes, W. N. Sloan, L. Ohno-Machado, and S. R. A. Deibel. A framework and tools for authoring, editing, documenting, sharing, searching, navigating, and executing computer-based clinical guidelines. In Proceedings of the American Medical Informatics Association Annual Fall Symposium, pages 261-265, 1999. 
[38] N. B. Guise, J. T. Huber, D. A. Giuse, C. W. Brown, B. A. Bankowitz, and S. Hunt. Information needs of health care professionals in an AIDS outpatient clinic as determined by chart review. Journal of the American Medical Informatics Association, 1:395-403, 1994.

[39] S. I. Herbert, C. J. Gordon, A. Jackson-Smale, and J.-L. R. Salis. Protocols for clinical care. Computer Methods and Programs in Biomedicine, 48(1-2):21-26, 1995.

[40] G. Hripcsak, P. Ludemann, T. A. Pryor, O. B. Wigertz, and P. Clayton. Rationale for the Arden syntax. Computers and Biomedical Research, 27(4):291-324, 1994.

[41] Impact Information Plain-Language Services. Plain language at work newsletter, March 2004. http://www.impact-information.com/impactinfo/newsletter/plwork08.htm (last accessed: May 07, 2009).

[42] InferMed. AREZZO. http://www.infermed.com/index.php/arezzo (last accessed: May 08, 2009).

[43] P. Johnson, S. W. Tu, M. A. Musen, and I. Purves. A virtual medical record for guidelinebased decision support. In Proceedings of the American Medical Informatics Association Annual Fall Symposium, pages 294-298, 2001.

[44] P. D. Johnson, S. W. Tu, N. Booth, B. Sugden, and I. N. Purves. Using scenarios in chronic disease management guidelines for primary care. In Proceedings of the American Medical Informatics Association Annual Fall Symposium, pages 389-393, 2000.

[45] R. B. Jones, J. Pearson, A. J. Cawsey, D. Bental, A. Barrett, J. White, C. A. White, and W. H. Gilmour. Effect of different forms of information produced for cancer patients on their use of the information, social support, and anxiety: randomised trial. British Medical Journal, 332:942-948, 2006.

[46] K. Kaiser, C. Akkaya, and S. Miksch. How can information extraction ease formalizing treatment processes in clinical practice guidelines? A method and its evaluation. Artificial Intelligence in Medicine, 39:151-163, 2007.

[47] K. Kaiser and S. Miksch. Modeling computer-supported clinical guidelines and protocols. A survey. Asgaard-TR-2005-2. Technical report, Vienna University of Technology, Institute of Software Technology and Interactive Systems, Austria, Vienna, 2005.

[48] K. Kaiser and S. Miksch. Versioning computer-interpretable guidelines: Semi-automatic modeling of 'living guidelines' using an information extraction method. Artificial Intelligence in Medicine, 46(1):55-66, 2009.

[49] C. Kerr, E. Murray, F. Stevenson, C. Gore, and I. Nazareth. Interactive health communication applications for chronic disease: patient and carer perspectives. Journal of Telemedicine and Telecare, 11(Supplement 1):32-34, 2005.

[50] B. M. Korsch, E. K. Gozzi, and V. Francis. Gaps in doctor-patient communication: I. Doctor-patient interaction and patient satisfaction. Pediatrics, 42(5):855-871, 1968.

[51] R. Kosara and S. Miksch. Metaphors of movement: A visualization and user interface for time-oriented, skeletal plans. Artificial Intelligence in Medicine, Special Issue: Information Visualization in Medicine, 22(2):111-131, 2001. 
[52] P. Ley. Giving information to patients. In J. R. Eisner, editor, Social Psychology and Behavioral Science, pages 339-365. John Wiley and Sons, NewYork, NY, 1982.

[53] D. F. Lobach, C. S. Gadd, and J. W. Hales. Structuring clinical practice guidelines in a relational database model for decision support on the internet. In Proceedings of the American Medical Informatics Association Annual Fall Symposium, pages 158-162, 1997.

[54] R. P. McArthur. From logic to computing. Wadsworth Publ. Co., Belmont, CA, 1991.

[55] D. McCaughan, C. A. Thompson, N. A. Cullum, T. A. Sheldon, and P. Raynor. Nurse practitioner and practice nurses' use of research information in clinical decision making: Findings from an exploratory study. Family Practice, 22:490-497, 2005.

[56] L. K. McKnight, P. D. Stetson, S. Bakken, C. Curran, and J. J. Cimino. Perceived information needs and communication difficulties of inpatient physicians and nurses. Journal of the American Medical Informatics Association, 9:64-69, 2002.

[57] Medical Dictionary. Online dictionary. http://medical-dictionary.thefreedictionary.com (last accessed: Sep 22, 2009).

[58] Medical Library Association. MLA - home page. http://www.mlanet.org/ (last accessed: Sep 22, 2009).

[59] C. Meredith, P. Symonds, L. Webster, D. Lamont, E. Pyper, C. R. Gillis, and L. Fallowfield. Information needs of cancer patients in west Scotland: Cross sectional survey of patients' views. British Medical Journal, 313(7059):724-726, 1996.

[60] G. Michel and R. Shiffman. GEM Cutter II user guide. Technical report, Yale Center for Medical Informatics, New Haven, Connecticut, 2007.

[61] M. A. Musen, S. W. Tu, A. K. Das, and Y. Shahar. EON: A component-based approach to automation of protocol-directed therapy. Journal of the American Medical Informatics Association, 3(6):367-388, 1996.

[62] J. Nielsen. The usability engineering life cycle. Computer, pages 12-22, 1992.

[63] D. E. Northup, M. Moore-West, B. Skipper, and S. R. Teaf. Characteristics of clinical information searching: Investigation using critical incident technique. Journal of Medical Education, 58:573-581, 1983.

[64] L. Ohno-Machado, J. H. Gennari, S. Murphy, N. L. Jain, S. W. Tu, D. E. Oliver, E. Pattison-Gordon, R. A. Greenes, E. H. Shortliffe, and O. Barnett. The guideline interchange format: A model for representing guidelines. Journal of the American Medical Informatics Association, 5(4):357-372, 1998.

[65] J. A. Osheroff, D. E. Forsythe, B. G. Buchanan, R. A. Bankowitz, B. H. Blumenfeld, and R. A. Miller. Physicians' information needs: Analysis of questions asked during clinical teaching. Annals of Internal Medicine, 514:576-581, 1991.

[66] M. Peleg, A. A. Boxwala, O. Ogunyemi, Q. Zeng, S. Tu, R. Lacson, E. Bernstam, N. Ash, P. Mork, L. Ohno-Machado, E. H. Shortliffe, and R. A. Greenes. GLIF3: The evolution of a guideline representation format. In Proceedings of the American Medical Informatics Association Annual Fall Symposium, pages 645-649, 2000. 
[67] M. Peleg, S. W. Tu, J. Bury, P. Ciccarese, J. Fox, R. A. Greenes, R. Hall, P. D. Johnson, N. Jones, A. Kumar, S. Miksch, S. Quaglini, A. Seyfang, E. H. Shortliffe, and M. Stefanelli. Comparing computer-interpretable guideline models: A case-study approach. Journal of the American Medical Informatics Association, 10(1):52-68, jan-feb 2003.

[68] K. A. Polvani, A. Agrawal, B. Karras, A. Deshpande, and R. Shiffman. GEM Cutter manual. Technical report, Yale Center for Medical Informatics, New Haven, Connecticut, 2000 .

[69] S. Quaglini, L. Dazzi, L. Gatti, M. Stefanelli, C. Fassino, and C. Tondini. Supporting tools for guideline development and dissemination. Artificial Intelligence in Medicine, 14 (1-2):119-137, 1998.

[70] S. Quaglini, M. Stefanelli, G. Lanzola, V. Caporusso, and S. Panzarasa. Flexible guidelinebased patient careflow systems. Artificial Intelligence in Medicine, 22:65-80, 2001.

[71] K. Richardson and S. Moran. Developing standards for patient information. International Journal of Health Care Quality Assurance, 8(7):27-31, 1995.

[72] M. Růžička and V. Svátek. Mark-up based analysis of narrative guidelines with the Stepper tool. In Computer-based Support for Clinical Guidelines and Protocols. Proceedings of the Symposium on Computerized Guidelines and Protocols, Studies in Health Technology and Informatics, volume 101, pages 132-136, Prague, Czech Republic, 2004. IOS Press.

[73] M. Růžička and V. Svátek. Stepper: Annotation and interactive stepwise transformation for knowledge-rich documents. In Engineering Knowledge in the Age of the Semantic Web, 14th International Conference, EKAW 2004, volume 3257 of Lecture Notes in Computer Science, pages 479-480, Whittlebury Hall, UK, October 5-8 2004. Springer Berlin / Heidelberg.

[74] S. M. Scheitel, B. J. Boland, P. C. Wollan, and M. D. Silverstein. Patient-physician agreement about medical diagnoses and cardiovascular risk factors in the ambulatory general medical examination. Mayo Clinic Proceedings, 71(12):1131-1137, 1996.

[75] M. Seeman and T. E. Seeman. Health behavior and personal autonomy: A longitudinal study of the sense of control in illness. Journal of Health and Social Behavior, 24:144-160, 1983.

[76] L. Selman, I. J. Higginson, G. Agupio, N. Dinat, J. Downing, L. Gwyther, T. Mashao, K. Mmoledi, A. P. Moll, L. M. Sebuyira, B. Panajatovic, and R. Harding. Meeting information needs of patients with incurable progressive disease and their families in South Africa and Uganda: Multicentre qualitative study. British Medical Journal, 338:b1326, 2009 .

[77] Y.-H. Seol, D. R. Kaufman, E. A. Mendonça, J. J. Cimino, and S. B. Johnson. Scenariobased assessment of physicians' information needs. In Proceedings from the Medinfo 2004 World Congress on Medical Informatics, volume 11, pages 306-310, 2004.

[78] A. Seyfang, S. Miksch, M. Marcos, J. Wittenberg, C. Polo-Conde, and K. Rosenbrand. Bridging the gap between informal and formal guideline representations. In G. Brewka, S. Coradeschi, A. Perini, and P. Traverso, editors, Proceedings of the 17th European Conference on Artificial Intelligence, pages 57-61. IOS Press, 2006. 
[79] Y. Shahar, S. Miksch, and P. Johnson. The Asgaard Project: A task-specific framework for the application and critiquing of time-oriented clinical guidelines. Artificial Intelligence in Medicine, 14(1-2):29-51, 1998.

[80] Y. Shahar, O. Young, E. Shalom, A. Mayaffit, R. Moskovitch, A. Hessing, and M. Galperin. DEGEL: A hybrid, multiple-ontology framework for specification and retrieval of clinical guidelines. In M. Dojat, E. Keravnou, and P. Barahona, editors, Proceedings of the 9th Conference on Artificial Intelligence in Medicine in Europe, volume 2780 of LNAI, pages 122-131, Protaras, Cyprus, 2003. Springer Verlag.

[81] R. N. Shiffman, B. T. Karras, A. Agrawal, R. Chen, L. Marenco, and S. Nath. GEM: A proposal for a more comprehensive guideline document model using XML. Journal of the American Medical Informatics Association, 7(5):488-498, 2000.

[82] R. N. Shiffman, G. Michel, A. Essaihi, and E. Thornquist. Bridging the guideline implementation gap: A systematic, document-centered approach to guideline implementation. Journal of the American Medical Informatics Association, 11(5):418-26, 2004.

[83] R. Smith. What clinical information do doctors need? British Medical Journal, 313: 1062-1068, 1996.

[84] R. Steele and J. Fox. Tallis PROforma primer - Introduction to PROforma language and software with worked examples. Technical report, Advanced Computation Laboratory, Cancer Research, London, UK, 2002.

[85] T. C. Strasser. The information needs of practising physicians in Northeastern New York State. Bulletin of the Medical Library Association, 66:200-209, 1978.

[86] V. Svátek and M. Růžička. Step-by-step mark-up of medical guideline documents. International Journal of Medical Informatics, 70:329-335, 2003.

[87] P. C. Tang. NetReach: A clinical information infrastructure for health-care teams in ambulatory care. Technical report, National Library of Medicine/High Performance Computing and Communications (NLM/HPCC) Contract No. N01-LM-4-3509, 1999.

[88] P. C. Tang, C. Newcomb, S. Gorden, and N. Kreider. Meeting the information needs of patients: Results from a patient focus group. In Proceedings of the American Medical Informatics Association Annual Fall Symposium, pages 672-676, 1997.

[89] R. L. Teach and E. H. Shortliffe. An analysis of physician attitudes regarding computerbased clinical consultation systems. Computers and Biomedical Research, 14:542-558, 1981.

[90] P. Terenziani, G. Molino, and M. Torchio. A modular approach for representing and executing clinical guidelines. Artificial Intelligence in Medicine, 23(3):249-276, 2001.

[91] The AGREE Collaboration. The appraisal of guidelines for research \& evaluation (AGREE) instrument, 2001. www. agreetrust.org (last accessed: May 18, 2009).

[92] The Dutch Institute for Healthcare Improvement. CBO - home page. http://www.cbo.nl (last accessed: July 21, 2009).

[93] The SAGE Project. Home page. http://sage.wherever.org/ (last accessed: May 18, 2009). 
[94] T. Timpka and E. Arborelius. The GP's dilemmas: A study of knowledge need and use during health care consultations. Methods of Information in Medicine, 29(1):23-29, 1990.

[95] T. Timpka, M. Ekstrom, and P. Bjurulf. Information needs and information seeking behavior in primary health care. Scandinavian Journal of Primary Health Care, pages 105-109, 1989.

[96] S. W. Tu and M. A. Musen. A flexible approach to guideline modeling. In Proceedings of the American Medical Informatics Association Annual Fall Symposium, pages 420-424, 1999.

[97] S. W. Tu, M. A. Musen, R. Shankar, J. Campbell, K. Hrabak, J. McClay, S. M. Huff, R. McClure, C. Parker, R. Rocha, R. Abarbanel, N. Beard, J. Glasgow, G. Mansfield, P. Ram, Q. Ye, E. Mays, T. Weida, C. G. Chute, K. McDonald, D. Molu, M. A. Nyman, S. Scheitel, H. Solbrig, D. A. Zill, and M. K. Goldstein. Modeling guidelines for integration into clinical workflow. Studies in Health Technology and Informatics, 107(1):174-178, 2004.

[98] S. Twaddle. Clinical practice guidelines. Singapore Medical Journal, 46(12):681-687, 2005.

[99] U.S. National Library of Medicine. Medical Subject Headings - home page. http://www.nlm.nih.gov/mesh/ (last accessed: May 23, 2009).

[100] P. Votruba, K. Kaiser, and S. Miksch. Tutorial for the document exploration and linking tool DELT/A. Technical report, Institute of Software Technology and Interactive Systems, Vienna University of Technology, Vienna, Austria, 2005. Annex of Protocure project deliverable D2.3a.

[101] P. Votruba, S. Miksch, and R. Kosara. Facilitating knowledge maintenance of clinical guidelines and protocols. In M. Fieschi, E. Coiera, and Y.-C. J. Li, editors, Proceedings from the Medinfo 2004 World Congress on Medical Informatics, pages 57-61. IOS Press, 2004 .

[102] B. Wallberg, H. Michelson, M. Nystedt, C. Bolund, L. F. Degner, and N. Wilking. Information needs and preferences for participation in treatment decisions among Swedish breast cancer patients. Acta Oncologica, 39(4):467-476, 2000.

[103] D. Wang, M. Peleg, S. W. Tu, A. A. Boxwala, R. A. Greenes, V. L. Patel, and E. H. Shortliffe. Representation primitives, process models and patient data in computer-interpretable clinical practice guidelines: A literature review of guideline representation models. International Journal of Medical Informatics, 68:59-70, 2002.

[104] J. W. Williamson, P. S. German, R. Weiss, E. A. Skinner, and F. Bowes. Health science information management and continuing education of physicians. A survey of US primary care practitioners and their opinion leaders. Annals of Internal Medicine, 110:151-160, 1989.

[105] S. H. Woolf and D. A. Benson. The medical information needs of internists and paediatricians at an academic medical centre. Bulletin of the Medical Library Association, 77: 372-380, 1989.

[106] X. Xu, G. D. Fiol, and R. A. Rocha. Towards a flexible web-based framework to navigate clinical reference documents. In Proceedings from the Medinfo 2004 World Congress on Medical Informatics, volume 2004, page 1915. IOS Press, Amsterdam, 2004. 
[107] X. Xu, R. A. Rocha, S. M. Bigelow, C. J. Wallace, T. Hanna, and L. K. Roemer. Understanding nurses' information needs and searching behavior in acute care settings. In Proceedings of the American Medical Informatics Association Annual Fall Symposium, pages 839-843, 2005.

[108] Q. Zeng and J. J. Cimino. Providing multiple views to meet physician information needs. Proceedings of the 33rd Hawaii International Conference on System Sciences, 5:5006, 2000. 